\title{
Elementary Particles, Dark Matter, and Dark Energy: Descriptions that Explain Aspects of Dark Matter to Ordinary Matter Ratios, Inflation, Early Galaxies, and Expansion of the Universe
}

\author{
Thomas J. Buckholtz
}

Affiliation: Ronin Institute, Montclair, New Jersey 07043, USA

E-mail: Thomas.Buckholtz@RoninInstitute.org

\begin{abstract}
Physics theory has yet to settle on specific descriptions for new elementary particles, for dark matter, and for dark energy forces. Our work extrapolates from the known elementary particles. The work suggests well-specified candidate descriptions for new elementary particles, dark matter, and dark energy forces. This part of the work does not depend on theories of motion. This work embraces symmetries that correlate with motion-centric conservation laws. The candidate descriptions seem to explain data that prior physics theory seems not to explain. Some of that data pertains to elementary particles. Our theory suggests relationships between masses of elementary particles. Our theory suggests a relationship between the strengths of electromagnetism and gravity. Some of that data pertains to astrophysics. Our theory seems to explain ratios of dark matter effects to ordinary matter effects. Our theory seems to explain aspects of galaxy formation. Some of that data pertains to cosmology. Our theory suggests bases for inflation and for changes in the rate of expansion of the universe. Generally, our work proposes extensions to theory in three fields. The fields are elementary particles, astrophysics, and cosmology. Our work suggests new elementary particles and seems to explain otherwise unexplained data.
\end{abstract}

Keywords: beyond the Standard Model, dark matter, dark energy, inflation, galaxy evolution, rate of expansion of the universe, quantum gravity, quantum field theory, mathematical physics, harmonic oscillator

Copyright (c) 2020 Thomas J. Buckholtz

\section{Contents}

1 Introduction $\quad \mathbf{3}$

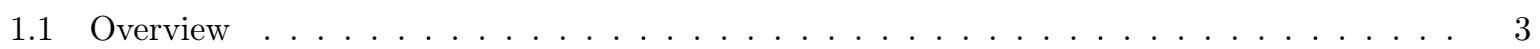

1.2 Context for and scope of proposed theory . . . . . . . . . . . . . . . . . . . . . 4

1.3 Similarities and differences between proposed theory and ongoing theory . . . . . . . . . 4

1.4 Nature, data, a framework for theories, theories and models, and mathematics . . . . . 4

1.5 Proposed theory, physics data, and ongoing theory . . . . . . . . . . . . . . 5

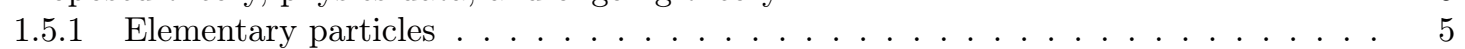

1.5.2 Dark energy forces and cosmology . . . . . . . . . . . . . . . . 6

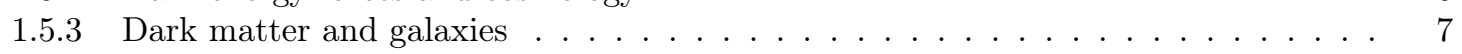

1.5 .4 Depletion of $\mathrm{CMB} \ldots \ldots \ldots \ldots \ldots \ldots$ 
1.5.5 Motion, kinematics conservation laws, QFT, QED, and QCD . . . . . . . 9

1.5.6 Kinematics and dynamics models . . . . . . . . . . . . . . . . . . . 9

1.5 .7 Other topics . . . . . . . . . . . . . . . . . . . 10

1.6 Evolution of proposed theory . . . . . . . . . . . . . . . . . . . 11

1.7 Perspective about this essay . . . . . . . . . . . . . . . . . . . 11

2 Elementary particles, field theories, and interaction vertices $\quad 12$

2.1 A table of elementary particles (or, simple particles and long-range forces) . . . . . . . . . 12

2.2 Theory that develops aspects of the table of simple particles and long-range forces . . . . 14

2.3 Kinematics conservation laws . . . . . . . . . . . . . . . . . . . . . 21

2.4 Long-range forces, including interactions with lepton number minus baryon number . . . . 23

2.5 Conservation of lepton number minus baryon number . . . . . . . . . . . . . . . . . . . 28

2.6 A table of subfamilies of simple particles and long-range forces . . . . . . . . . . . . . . . 29

2.7 Bases for proposed field theories . . . . . . . . . . . . . . . . . . . . . . . . 29

2.8 Interaction vertices . . . . . . . . . . . . . . . . . . . . . . . . 33

2.9 Aspects regarding simple particles . . . . . . . . . . . . . . . . . . . . . 34

2.10 Masses of the weak interaction bosons, the Higgs boson, and the OI boson . . . . . . . . . 35

2.11 A prediction for the tauon mass . . . . . . . . . . . . . . . . . . 36

2.12 The relative strengths of electromagnetism and gravity . . . . . . . . . . . . . . . . . 37

2.13 The masses of quarks and charged leptons . . . . . . . . . . . . . . . . . . . . . . . . . . . . . . . . . . . .

2.14 Neutrino masses . . . . . . . . . . . . . . . . . . . . . . . . . . . . 39

2.15 Anomalous moments . . . . . . . . . . . . . . . . . . . . . . . . . . . 40

2.16 Possible threshold energies for observing effects of tweak (or, 2T) simple particles . . . . 42

2.17 Possibilities for detecting or inferring aye (or, 0I) simple particles . . . . . . . . . . . . . 43

2.18 Relationships between ALG modeling, PDE modeling, kinematics modeling, and physics . 44

3 Dark matter, dark energy, astrophysics, and cosmology $\quad \mathbf{4 5}$

3.1 Models that have bases in one, six, and 36 isomers of charge . . . . . . . . . . . . . . . 45

3.2 Spans for objects and long-range forces . . . . . . . . . . . . . . . . . . . . . . 46

3.3 Densities of the universe . . . . . . . . . . . . . . . . . . . . . . . 50

3.4 Dark matter ratios inferred from data regarding cosmic microwave background radiation . 51

3.5 The rate of expansion of the universe . . . . . . . . . . . . . . . . . . . 5 51

3.6 Phenomena during and just after inflation . . . . . . . . . . . . . . . . . . . . 52

3.7 Baryon asymmetry . . . . . . . . . . . . . . . . . . . . . . . 53

3.8 Galaxies, components of galaxies, and ratios of dark matter to ordinary matter . . . . . . 54

3.9 Galaxy clusters, ratios of dark matter effects to ordinary matter effects, and filaments . . 58

3.10 Dark energy density . . . . . . . . . . . . . . . . . . . . . . 58

3.11 High-mass neutron stars . . . . . . . . . . . . . . . . . . . . . . . . 59

3.12 Phenomena before inflation . . . . . . . . . . . . . . . . . . . 60

3.13 Directly detecting dark matter and doubly dark matter . . . . . . . . . . . . . . . 60

4 Relationships between various theories and models for motion $\mathbf{6 0}$

4.1 Kinematics modeling and dynamics modeling . . . . . . . . . . . . . . . . . . . 61

4.2 Perspective regarding quantum modeling and kinematics modeling . . . . . . . . . . . . . 62

4.3 Possible complements to ongoing theory QFT, QED, and QCD . . . . . . . . . . . 62

4.4 Dynamics models for hadron-like particles . . . . . . . . . . . . . . . . . . 63

4.5 Dynamics models for nuclear physics . . . . . . . . . . . . . . . . . . . . . . . . 64

4.6 Dynamics models for quantum transitions . . . . . . . . . . . . . . . . . . . . . . 64

4.7 Models for interactions with gravity . . . . . . . . . . . . . . . . . . . . . 65

4.8 General relativity and large-scale physics . . . . . . . . . . . . . . . . . . . . . 65

4.9 Aspects of dynamics modeling regarding hadron-like particles . . . . . . . . . . . . . . 66

4.10 Possible modeling for fissionable or bound-state multicomponent objects . . . . . . . . . . 67

4.11 Modeling regarding refraction and similar effects . . . . . . . . . . . . . . . . . 68

4.12 U-family interactions and the strong interaction $S U(3)$ symmetry $\ldots \ldots \ldots$ 
5 Relationships between various theories and models for objects $\quad \mathbf{7 0}$

5.1 The elementary particle Standard Model . . . . . . . . . . . . . . . . . . 70

5.2 The strong CP problem and possible axion elementary particles . . . . . . . . . . . . 71

5.3 Channels and G-family interactions . . . . . . . . . . . . . . . . . . . . . 71

5.4 Lack of magnetic monopoles and a possible lack of some electric dipole moments . . . . 72

5.5 Supersymmetry . . . . . . . . . . . . . . . . . . . . . . . . 72

5.6 String theory . . . . . . . . . . . . . . . . . . . . . . . . 72

5.7 Other discussion regarding the masses of simple particles and hadron-like particles . . . . 73

5.8 The cosmology timeline . . . . . . . . . . . . . . . . . . . . . . . 74

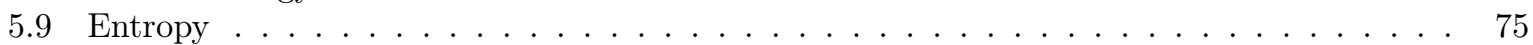

6 Possible opportunities to develop deeper insight $\quad \mathbf{7 6}$

6.1 Numbers of dimensions . . . . . . . . . . . . . . . . . . . . . . . . 76

6.2 A series of formulas for lengths, including the Planck length . . . . . . . . . . . . . 76

6.3 CPT-related symmetries . . . . . . . . . . . . . . . . . . . . . . 77

6.4 Relationships between properties of objects and forces . . . . . . . . . . . . . . . . . . . . . . . . . . .

6.5 Arrow of time and entropy . . . . . . . . . . . . . . . . . . . . . 78

6.6 The Higgs mechanism, entanglement, and tachyon-like behavior . . . . . . . . . . . . . 79

6.7 Notions regarding possible universes beyond our universe . . . . . . . . . . . . . . . . 79

6.8 Notions that might link physics constants and modeling . . . . . . . . . . . . . . . . 79

$\begin{array}{llr}7 & \text { Concluding remarks } & 80\end{array}$

$\begin{array}{lr}\text { Acknowledgments } & 81\end{array}$

$\begin{array}{lr}\text { References } & 81\end{array}$

\section{Introduction}

This unit provides an overview of theory that this essay proposes. This unit discusses context and scope for the proposed theory. This unit discusses relationships between data, proposed theory, ongoing theory, and mathematics. This unit discusses evolution of proposed theory. This unit provides perspective about this essay.

\section{$1.1 \quad$ Overview}

This unit discusses highlights about proposed theory.

We develop a framework for physics theories. The framework seems to embrace ongoing theories. The framework suggests new physics theory. We use the two-word term proposed theory to denote the new theory.

The following items point to topics that proposed theory addresses. Each item notes aspects that underlie the framework or come from proposed theory.

- Extensions to harmonic-oscillator mathematics. Minor changes in assumptions lead to states that people might consider to lie below traditional ground states. The resulting math has applications to elementary particle physics.

- Mathematics-based modeling pertaining to elementary particles, astrophysics, and cosmology. Extended harmonic-oscillator math provides bases for modeling pertaining to elementary particles, dark matter, dark energy forces, and observations that physicists report. Modeling features solutions to equations featuring isotropic pairs of isotropic quantum harmonic oscillators.

- Elementary particles. A model outputs solutions correlating with all known elementary particles and a list of suggested elementary particles.

- Astrophysics (dark matter). A combination of the list of elementary particles and one additional assumption suggests a well-specified candidate description for dark matter. That description explains various observed ratios of dark matter effects to ordinary matter effects. 
- Cosmology. The list of elementary particles includes a description of dark energy forces. The description of dark energy forces explains eras regarding the rate of expansion of the universe. The list of elementary particles provides a specification for an inflaton. The inflaton provides candidate explanations for aspects of inflation and of phenomena immediately after the inflationary epoch. The list of elementary particles provides a candidate explanation for baryon asymmetry.

- Astrophysics (galaxy formation). A combination of dark matter aspects and dark energy forces leads to galaxy formation scenarios that match observed data.

- Fundamental aspects of physics. The work relates a ratio of the masses of two elementary particles to a ratio of the strength of electromagnetism to the strength of gravity. Other formulas may interrelate the masses of other elementary particles.

\subsection{Context for and scope of proposed theory}

This unit discusses context for, aspects of, and the scope of proposed theory.

Physics includes issues that have remained unresolved for decades. For one example, describe elementary particles that people have yet to find. For another example, describe dark matter. For another example, describe dark energy pressure or forces. For each of those examples, resolution does not necessarily depend on considering models pertaining to translational motion.

Ongoing theory has bases in developing theories of motion without necessarily having descriptions of objects that move. Examples of such theories feature epicycles, elliptical orbits, and the principle of stationary action. Ongoing theory has bases in adding quantization to classical modeling of the motion of objects.

We pursue an approach that catalogs fundamental objects and their properties. The approach features, from its beginning, quantized concepts. The approach does not originally address translational motion.

The approach matches, explains, or predicts phenomena that ongoing theory approaches do not. For example, we suggest - with some specificity - descriptions of new elementary particles, dark matter, and dark energy forces. The approach suggests formalism that can complement and integrate with ongoing theory.

\subsection{Similarities and differences between proposed theory and ongoing theory}

This unit notes some similarities and some differences between proposed theory and ongoing theory.

Physics theories entwine concepts. One concept is that of an object. One concept features internal properties of objects. One concept features motions of objects. One concept features interactions between objects. One concept features changes over time regarding objects, their internal properties, their motions, and their interactions.

The Newtonian physics equation $\vec{F}=m \vec{a}$ embraces each one of those five concepts. The equation applies to one object. The term $m$ refers to an internal property, mass, of the object. The term $\vec{a}$ refers to acceleration and dovetails with motion. The term $\vec{F}$ denotes force and refers to interactions between the object and other objects. At least one term, $\vec{a}$, pertains regarding changes over time. One change over time pertains to velocity. Velocity is an aspect of motion.

Each one of ongoing theory and proposed theory addresses all of the five concepts.

Roots for ongoing theory include models for motion, models for interactions, and mathematics that features continuous functions. People use the four-word term principle of stationary action to name one guiding tenet.

Roots for proposed theory include models for objects, models for internal properties, models for interactions, and mathematics that features discrete solutions. We use the two-element term doubleentry bookkeeping to name one guiding tenet.

Proposed theory tries to address topics that ongoing theory tries to address. (For example, reference [1] provides an ongoing theory attempt to address topics that proposed theory tries to address.)

\subsection{Nature, data, a framework for theories, theories and models, and math- ematics}

This unit interrelates concepts regarding nature, data, theories and modeling, and mathematics.

Physics entwines concepts. One concept is that of nature. One concept is data about nature. One concept is models that explain or predict data. One concept is theories that underlie models. One concept 
is mathematics that underlies theories or models. The concepts do not necessarily separate completely. Interpretations of data depend on models.

We show and use a framework that encompasses some physics theories. The framework encompasses some ongoing theory. The framework encompasses the proposed theory that we suggest.

We extend traditional mathematics for harmonic oscillators. The extended math provides a basis for the framework. The extended math provides a basis for theories and models that we suggest.

We use the framework to spot choices regarding theories and models. We discuss possible synergies between some theories. We discuss possible discord between some theories. We discuss possible synergies between some models. We discuss possible discord between some models.

We explore the range of applicability for each of some theories and for each of some models. We prefer to correlate a range with data. We sometimes correlate a range with theories or models. We de-emphasize some notions such as the notion that nature, in effect, understands or follows mathematics correlating with specific theories or models.

\subsection{Proposed theory, physics data, and ongoing theory}

This unit provides an overview of proposed theory. This unit discusses relationships between proposed theory, physics data, and ongoing theory.

Generally, proposed theory suggests complements (or, additions) to ongoing theory. We suggest additions to the list of elementary particles. We suggest descriptions for dark matter and for dark energy forces. We suggest new symmetries and, therewith, at least one new conservation law. We suggest new approximate symmetries and, therewith, new somewhat conservation laws. Some of our suggestions point to possibilities for new interpretations regarding known data.

Generally, proposed theory tends to rely on ongoing theory concepts regarding objects, internal properties of objects, motion-centric properties, interactions, and kinematics and dynamics theories. Some proposed theory offers complements to or suggests limits regarding some aspects of ongoing theory kinematics and dynamics modeling.

Nearby below, we summarize some aspects of and results from proposed theory. We provide perspective for understanding, evaluating, and using proposed theory. We discuss overlaps, similarities, differences, possible synergies, and possible conflicts between proposed theory, physics data, and some aspects of ongoing theory.

\subsubsection{Elementary particles}

This unit summarizes - regarding elementary particles - aspects of and relationships between proposed theory, physics data, and ongoing theory.

People try two approaches to suggesting new elementary particles. People try to explain observed phenomena by suggesting new elementary particles. Perhaps, dark matter has bases in WIMPs or axions. Perhaps, gravity correlates with gravitons. Perhaps, some possible violation of CP symmetry suggests that nature includes axions. People try to determine patterns that would suggest new particles. Perhaps, supersymmetry pertains and predicts new elementary particles.

Explaining phenomena has succeeded in the past. Explaining protons led to predicting and discovering quarks. Explaining, within the context of gauge theory, the non-zero masses of the $\mathrm{W}$ and $\mathrm{Z}$ bosons led to predicting and discovering the Higgs boson.

Proposing patterns has succeeded in the past. The proposing, in 1869 by Mendeleev, of organizing principles related to properties of chemical elements led to the periodic table for elements. (Note reference [2].) The table matched all the then-known elements and suggested elements that people subsequently discovered.

Physics might benefit from new candidates for sets of organizing principles for elementary particles. Currently, ongoing theory sets of candidate principles (such as principles that correlate with supersymmetry) seem to be unverified or to lack specificity regarding properties of particles.

Proposed theory includes a mathematics-based modeling technique that, in effect, outputs the list of known elementary particles, suggests new elementary particles, and suggests organizing principles for an elementary particle analog to the periodic table for chemical elements. The modeling technique does not require making a choice among ongoing theory kinematics theories.

We think that the set of candidate elementary particles explains some and perhaps most or all of the phenomena that people currently consider when people use known phenomena to point to the possible existence of new elementary particles. Examples of those phenomena include dark matter and baryon asymmetry. 
While one mathematics modeling basis outputs the entire set of known and suggested elementary particles, we find it convenient to divide the set into two subsets. We use the two-word phrase simple particles to point to all of the aspects except long-range forces. We use the two-element phrase long-range forces to include bases for phenomena such as electromagnetic fields, gravity, and dark energy forces. We do not separate the notion of boson particles from a broader (than just long-range) concept of forces. For example, sometimes, modeling based on the notion of a strong force provides advantages over modeling based on the notion of gluon simple particles.

We think that people can use the set of elementary particles in the context of ongoing theory classical physics and in the context of ongoing theory quantum physics. We think that people can use the set of elementary particles in the contexts of modeling based on each of Newtonian kinematics, special relativity, and general relativity.

People might treat outputs from the modeling technique as candidates for simple particles and longrange forces. Some or all of the candidates might represent opportunities for research to detect or infer phenomena. The candidates might not necessarily conflict with verified aspects of ongoing theory.

\subsubsection{Dark energy forces and cosmology}

This unit summarizes - regarding dark energy forces and cosmology - aspects of and relationships between proposed theory, physics data, and ongoing theory.

People suggest the concept of dark energy pressure to explain observed changes in the rate of expansion of the universe. Ongoing theory concepts that people use to try to model aspects of the rate of change include the Hubble parameter (or, Hubble constant), equations of state (or, relationships between density and pressure), and general relativity. People suggest possible incompatibilities between observations and ongoing theory modeling. (See, for example, references [3], [4], and [5]. However, some people note possible objections to some notions of incompatibility. See, for example, references [6] and [7].) People suggest phenomenological remedies regarding the modeling. (See, for example, reference [8].) People sometimes use the three-word term dark energy forces in discussions that include notions of dark energy pressure.

Each of proposed theory and ongoing theory uses terms such as gravity and gravitation. Proposed theory includes concepts of components of long-range forces. For example, regarding electromagnetism, one component correlates with interactions with charge and another component correlates with interactions with nominal magnetic dipole moment. The existence of these components is appropriate because relevant modeling does not necessarily correlate with translational motion. Some objects have non-zero charge and zero nominal magnetic dipole moment. Some objects have zero charge and non-zero nominal magnetic dipole moment. Ongoing theory sometimes decomposes aspects of electromagnetism into an electric field and a magnetic field. However, ongoing theory notions of photons do not necessarily reflect such a decomposition. Similar concepts pertain regarding gravity. Proposed theory uses the sixword term basic gravity and dark energy forces (or, basic gravity plus dark energy forces). The basic gravity component of gravitation correlates well with a Newtonian notion of gravity associated with a non-rotating object that models as existing at a point or as having spherical symmetry. The dark energy forces components correlate with - among other phenomena - changes in the rate of expansion of the universe. An example of such other phenomena features aspects correlating with gravity associated with a rotating spherically symmetric object. Ongoing theory does not necessarily similarly decompose notions of gravity. For example, general relativity does not necessarily try to separate notions that might correlate with basic gravity from notions that might correlate with dark energy forces.

Proposed theory regarding spin-two long-range forces points to a candidate unified treatment of basic gravity and dark energy forces. Proposed theory provides a candidate explanation for observed eras in the rate of expansion of the universe. The first era correlates with a rate that increases with time. We characterize the dominant force components for this era by the word octupole. The dominant force components repel objects from each other. The second era correlates with a rate that decreases with time and, if we assume data that references [9], [10], [11], and [12] provide, that ends some billions of years later. We characterize the dominant force component for this era by the word quadrupole. The dominant force component attracts objects to each other. The third era correlates with a rate that increases with time and has lasted some billions of years. We characterize the dominant force component for this era by the word dipole. The dominant force component repels objects from each other. For each era, dominance refers to interactions between somewhat similar large neighboring objects. Interactions between smaller neighboring objects transit, generally comparatively quickly, to dominance by a monopole force, namely basic gravity. 
We correlate with the three-word term dark energy forces the spin-two octupole, quadrupole, and dipole long-range forces that we just mentioned.

We think that proposed theory provides a candidate means to close gaps between observations and ongoing theory. Opportunities exist to characterize (in terms of the rest energies and a few other characteristics, such as rates of rotation, of objects) the strengths of the non-monopole force components of our suggested notion of basic gravity plus dark energy forces. We think that proposed theory is not incompatible with the ongoing theory notion that the Einstein field equations can be compatible with repulsion. (See reference [13].)

\subsubsection{Dark matter and galaxies}

This unit summarizes - regarding dark matter and galaxies - aspects of and relationships between proposed theory, physics data, and ongoing theory.

People suggest various explanations for observations that, starting in the 1880s, suggest that the Milky Way galaxy does not have enough ordinary matter to keep observed stars in their orbits; that, starting in the 1930s, suggest that galaxy clusters do not contain enough ordinary matter to bind observed galaxies into the clusters; and that, starting in the 1930s, suggest that a significant fraction of observed galaxies do not have enough ordinary matter to keep observed stars in their orbits. While people discuss theories that might not require nature to include dark matter, most observations and theoretical work assume that dark matter exists. (People use the term MOND - or, modified Newtonian dynamics - to describe one set of theories that might obviate needs to assume that nature includes dark matter.) People use terms such as WIMPs (or, weakly interacting massive particles), axions, and primordial black holes to name candidate explanations for dark matter. Some of the candidates are not necessarily well-specified. For example, searches for axions span several orders of magnitude of possible axion mass. People suggest that nature might include dark matter photons. (See, for example, reference [14].) People suggest that dark matter might include components that include quarks or that might experience Yukawa-like potentials. (See, for example, references [15] and [16].)

Proposed theory suggests that nature includes objects that behave like WIMPs but are not elementary particles. These objects would be similar to protons, neutrons, and other hadrons, except that the quark-like components would be fermion elementary particles that have zero charge. These hadron-like particles would interact with gravity. These hadron-like particles would have no non-zero-charge internal components and would not interact with light. We know of no reason why these particles would be incompatible with ongoing theory.

Assuming that the WIMP-similar hadron-like particles exist in nature, a question remains as to the extent to which these particles comprise all dark matter. We think that, today, ongoing theory would not resolve that question.

People infer a ratio of dark matter density of the universe to ordinary matter density of the universe. That ratio is five-plus to one. (See data that reference [17] provides.) People also infer ratios, for some galaxies and for some galaxy clusters, of dark matter effects to ordinary matter effects.

We think that ongoing theory does not provide bases for explaining, from fundamental principles, those observed ratios.

Proposed theory includes three cases. We denote the cases by symbols of the form PRnISe. The three relevant values of $\mathrm{n}$ are one, six, and 36. The symbol PR abbreviates the one-element term physicsrelevant. The symbol ISe abbreviates the four-word phrase isomers of the electron. For each case, we assume that nature embraces $\mathrm{n}$ isomers of charged simple particles. We assume that each isomer of charged simple particles interacts, via charge and nominal magnetic dipole moment, with its own isomer of so-called PR1ISe-like photons. For n equal to six and n equal to 36, each isomer of charged simple particles does not interact, via charge and nominal magnetic dipole moment, with isomers, other than its own isomer, of PR1ISe-like photons. We introduce the word span. We say that the span of each isomer of charged simple particles is one, as in one isomer of charged simple particles. The span of each isomer of PR1ISe-like photons is one, as in one isomer of charged simple particles. For $\mathrm{n}$ equal to six, one isomer of basic gravity interacts with all six isomers of charged simple particles. We say that the span of basic gravity is six, as in six isomers of charged simple particles. (A span of six does not pertain regarding dark energy forces. For example, the span for each of six isomers of the quadrupole component of dark energy forces is one.) One isomer of charged simple particles correlates with ordinary matter. The other five isomers of charged simple particles correlate with dark matter. For n equal to 36, nature embraces six isomers of basic gravity, with each isomer of basic gravity correlating with its own PR6ISe-like set of phenomena. One PR6ISe-like set correlates with ordinary matter plus dark matter. We use the threeword term doubly dark matter to refer to the 30 isomers of charged simple particles that are neither 
ordinary matter nor dark matter.

PR1ISe does not provide bases for explaining, from fundamental principles, observed ratios of dark matter effects to ordinary matter effects.

PR6ISe provides bases for explaining some observed ratios. For example, regarding densities of the universe, the five dark matter isomers explain the five in the ratio five-plus to one. The WIMP-similar hadron-like particles explain the plus in the ratio five-plus to one. For example, PR6ISe seems to explain galaxy-related observed ratios.

PR36ISe provides the same bases (that PR6ISe provides) for explaining the above-mentioned observed ratios. PR36ISe differs from PR6ISe regarding explaining a possibly observed ratio that correlates with depletion of cosmic microwave background radiation.

Each of the cases PR6ISe and PR36ISe suggests the following scenario for the formation and evolution of galaxies. For purposes of discussion, we de-emphasize PR36ISe.

The scenario features, for each galaxy, the notion of an original clump. Clumping takes placed based on the quadrupole long-range force, which is attractive and has a span of one, as in one isomer of charged simple particles. For each of many galaxies, the initial clump correlates with one isomer of PR6ISe-spanone phenomena. Sometimes, an original clump features, based on the attractive monopole long-range force, more than one isomer of PR6ISe-span-one phenomena. With respect to each isomer in the clump, the repulsive dipole long-range force drives away from the original clump one isomer of PR6ISe-span-one phenomena. Thus, for essentially all galaxies, the original clump correlates with no more than three isomers of PR6ISe-span-one phenomena.

From a standpoint of observations, three types of one-isomer original clump galaxies exist. One-sixth of one-isomer original clump galaxies feature an ordinary matter original clump. Two-thirds of one-isomer original clump galaxies feature a dark matter original clump that does not repel ordinary matter. Onesixth of one-isomer original clump galaxies feature a dark matter original clump that repels ordinary matter. We suggest that the ongoing theory notion of dark matter galaxy correlates with galaxies for which dark matter original clumps repel ordinary matter.

Observations of early galaxies correlate with galaxies for which the original clump contains significant amounts of ordinary matter. Aside from dark matter galaxies, galaxies for which the original clump features just one isomer of PR6ISe-span-one phenomena might attract and accumulate matter such that eventually (assuming that disturbances, such as collisions with other galaxies, do not occur) the galaxies contain approximately four times as much dark matter that has bases in PR6ISe-span-one phenomena as ordinary matter.

We think that data supports the galaxy formation and evolution scenario. Reference [18] discusses a dark matter galaxy. Reference [19] reports, regarding galaxies about 10 billion years ago, data that seems to support the notion of ordinary matter intensive original clumps. Figure 7 in reference [20] seems to support (especially via data pertaining to redshifts of at least seven) the notion of ordinary matter intensive original clumps. Observations that reference [21] reports might support the notion of an approximately four to one ratio that might correlate with the approximately five-sixths of one-isomer clump galaxies that are not dark matter galaxies. The observation that reference [22] reports might correlate with a three-isomer original clump galaxy.

The galaxy formation and evolution scenario seems to comport with data. For each of many known galaxies, the scenario is not necessarily incompatible with the ongoing theory notion of a dark matter halo. The scenario does not necessarily comport, for some ordinary matter intense galaxies, with some ongoing theory assumptions about roles, in galaxy formation, of dark matter halos.

We think that each of PR6ISe and PR36ISe is not incompatible with inferred galaxy cluster related ratios of dark matter effects to ordinary matter effects.

Each of PR6ISe and PR36ISe seems to offer an explanation for one piece of data regarding details of the Milky Way galaxy. (Regarding the piece of data, see discussion, in reference [23], regarding data regarding the stellar stream GD-1.)

\subsubsection{Depletion of CMB}

This unit summarizes - regarding one observation of depletion of cosmic microwave background radiation - aspects of and relationships between proposed theory, physics data, and ongoing theory.

Results that reference [24] reports about depletion of CMB (or, cosmic microwave background radiation) by absorption by hydrogen atoms might dovetail with the existence of dark matter isomers of hydrogen atoms or with the existence of doubly dark matter isomers of hydrogen atoms. For the PR6ISe case, dark matter pertains. For the case PR36ISe, doubly dark matter pertains. Proposed theory might contribute to credibility for assumptions and calculations that led to the prediction for the amount of 
depletion that correlates with ordinary matter hydrogen atoms. (Regarding the assumptions and calculations, see reference [25].)

\subsubsection{Motion, kinematics conservation laws, QFT, QED, and QCD}

This unit summarizes - regarding motion, kinematics conservation laws, QFT (or, quantum field theory), QED (or, quantum electrodynamics), and QCD (or, quantum chromodynamics) - aspects of and relationships between proposed theory, physics data, and ongoing theory.

Ongoing theory has roots in theories of motion. Aspects, to which we allude above, of proposed theory generally do not depend on choosing a specific model regarding translational motion.

Ongoing theory correlates an $S U(2)$ symmetry with conservation of angular momentum and correlates an $S U(2)$ symmetry with conservation of (linear) momentum.

Proposed theory permits adding, to work to which we allude above, ongoing theory symmetries correlating with conservation of angular momentum and conservation of linear momentum. We can add symmetry correlating with conservation of energy. Regarding models that correlate with special relativity, we can add symmetry correlating with boost.

Regarding conservation of angular momentum and conservation of linear momentum, proposed theory permits either of two choices. For one choice, one can add, for each of simple fermions and elementary bosons, two $S U(2)$ symmetries. This choice provides a path toward much ongoing theory QFT, QED, and QCD. For the other choice, one can add one $S U(2)$ symmetry for simple fermions and one $S U(2)$ symmetry for elementary bosons. This proposed theory choice provides an alternative, to ongoing theory means, way for modeling aspects of dynamics within multiparticle systems such as protons. Here, kinematics conservation laws pertain for the proton but do not necessarily pertain for individual components of the proton. Modeling correlating with special relativity can pertain for the proton without pertaining to individual components of the proton. Modeling based on potentials can pertain. Modeling does not necessarily feature elementary bosons or virtual particles.

Mathematics-modeling bases for proposed theory QFT, QED, and QCD are inherent in the mathematicsmodeling bases that underlie aspects of proposed theory that emphasize objects and (up to now in this discussion) de-emphasize motion. The bases include aspects that correlate with ongoing theory concepts of fields and particles. The bases include aspects that correlate with interaction vertices that are volumelike with respect to coordinates. The volume-like aspects correlate, for example, with the concept that one can model, for a proton, one quark as existing within a potential correlating with the other two quarks. Modeling for a proton suggests that boost symmetry (and some alternatives, including no symmetry) which might pertain for the proton - correlates with modeling that would (had modeling via potentials not, in effect, replaced modeling via virtual elementary bosons) be related to gluons.

Proposed theory QFT, QED, and QCD offer some advantages and exhibit some possible disadvantages compared to ongoing theory QFT, QED, and QCD. Aspects of proposed QED and QCD may be less complex conceptually and more sound mathematically than similar aspects of ongoing QED and QCD. Proposed QED modeling and proposed QCD modeling do not necessarily involve the concept of virtual particles. Aspects of proposed QED and QCD may be less developed and less capable of producing without results from observations or from ongoing theory - numerical results than are similar to aspects of ongoing QED and QCD.

We think that proposed QED and QCD and ongoing QED and QCD do not conflict significantly with each other and might provide synergies between each other.

\subsubsection{Kinematics and dynamics models}

This unit summarizes - regarding kinematics and dynamics models - aspects of and relationships between proposed theory, physics data, and ongoing theory.

Ongoing theory provides choices regarding bases for kinematics and dynamics models. One choice features quantum physics modeling and classical physics modeling. Another choice features Newtonian physics, special relativity, general relativity, and other possible bases.

We think that the set of simple particles and long-range forces that proposed theory suggests is compatible with ongoing theory choices regarding kinematics and dynamics models that we list above, except possibly regarding some modeling that would be based on general relativity. Ongoing theory seems open to the concept that general relativity might not pertain well for some large-scale aspects of nature. (See, for example, reference [26].)

Modeling based on general relativity might not be adequately accurate to the extent that some adequately significant phenomena correlate with one span and other adequately significant phenomena 
correlate with another span. For example, regarding PR6ISe modeling under circumstances in which the quadrupole attractive component of dark energy forces dominates, a dark matter clump that starts on a trajectory similar to the trajectory of a similar ordinary matter clump would not necessarily follow the trajectory that the ordinary matter clump follows. The isomer of the quadrupole attractive component of dark energy forces that correlates with the ordinary matter clump does not equal the isomer of the quadrupole attractive component of dark energy forces that correlates with the dark matter clump. While all six isomers of PR6ISe-span-one phenomena interact via monopole gravity (or, basic gravity), each one of the six isomers of PR6ISe-span-one phenomena interacts with itself, but not with other isomers of PR6ISe-span-one phenomena, via the quadrupole and octupole components of dark energy forces. Reference [8] points to a possible difficulty regarding modeling based on general relativity. We suggest that this possible difficulty might correlate with aspects of dark energy forces.

We think that proposed theory is not incompatible with known observations that people correlate with validating general relativity. Opportunities might exist to determine the extent to which proposed theory extends applications of general relativity to some realms for which people have not verified the applicability of general relativity. For example, our dipole component of dark energy forces might correlate with the ongoing theory general relativity concept of rotational frame-dragging.

\subsubsection{Other topics}

This unit summarizes - regarding various topics - aspects of and relationships between proposed theory, physics data, and ongoing theory.

Regarding proposed theory, people might assume that the following aspects are non-traditional or think that the following aspects are controversial. However, we think that proposed theory shows that these aspects comport with known phenomena, do not contradict known phenomena, do not violate ongoing theory theories for realms in which people have validated the theories, offer ways to strengthen and further understand some ongoing theory, and offer parallel theories that are synergistic with ongoing theory.

- Proposed theory points to a formula that possibly links a ratio of the masses of two elementary particles and a ratio of the strengths of two components of long-range forces. The elementary particles are the tauon and the electron. The forces are electrostatic repulsion between two electrons and gravitational attraction between (the same) two electrons. We think that this numeric relationship comports with measurements and points to a possibility for extending physics theory. The formula suggests a tauon mass and a standard deviation for the tauon mass. Based on 2018 data, four calculated standard deviations fit within one experimental standard deviation of the experimental nominal tauon mass.

- Proposed theory points to (at least approximate) numerical relationships between the ratios of the masses of the Higgs, Z, and W bosons. These relationships might suggest possibilities for extending physics theories related to the weak mixing angle.

- Proposed theory suggests that people might be able to distinguish observationally between the coalescing of two black holes that interact with each other via dark energy force dipole repulsion and the coalescing of two black holes that do not interact with each other via dark energy force dipole repulsion.

- Proposed theory suggests resolution regarding the possible mismatch between the elementary particle Standard Model notion that all neutrinos might have zero rest mass and interpretations, of data, that people associate with the notion that at least one neutrino flavor (or, generation) has non-zero rest mass. Proposed theory suggests that spin-four components of long-range forces couple to lepton number (and not to rest mass) and underlie phenomena that people interpret as implying that at least one neutrino has non-zero rest mass. Proposed theory suggests that ongoing theories might interpret those phenomena as correlating with a specific value for a would-be sum of neutrino rest masses. That value is $3 \alpha^{2} m_{e}$. The symbol $\alpha$ denotes the fine-structure constant. The symbol $m_{e}$ denotes the mass of an electron. That value comports with ongoing theory interpretations of data, as summarized by reference [17]. Proposed theory suggests that all neutrinos might have zero rest mass. While this work may prove controversial, we offer the possibility that it comports with data and resolves an underlying tension regarding ongoing theory.

- Proposed theory suggests details about simple particles and long-range forces involved during the inflationary epoch. 
- Proposed theory QED points to a possibility for modeling lepton anomalous magnetic dipole moments via a sum of three terms. Each term correlates with a component, for which the spin exceeds one, of long-range forces. This work exemplifies remarks above about relative advantages of, relative disadvantages of, and possible synergies between proposed theory QED and ongoing theory QED.

- We think that possibilities exist for adding, to the elementary particle Standard Model, new elementary particles that proposed theory suggests. Some of the new elementary particles correlate with symmetries that correlate with current Standard Model elementary particles. Examples include two new non-zero-mass spin-one simple bosons, which would correlate with an $S U(2) \times U(1)$ symmetry similar to the symmetry correlating with the $\mathrm{W}$ and $\mathrm{Z}$ bosons. So far, proposed theory does not fully explore the feasibility of adding, to the Standard Model, the particles that proposed theory suggests. For example, we do not explore Lagrangian terms for candidate particles. Also, we do not explore the extent of compatibility between the Standard Model and PR6ISe modeling.

- Proposed theory suggests possibilities for a new look at aspects of nuclear physics. Proposed theory that suggests new elementary particles suggests one elementary particle that might correlate with repulsive aspects of the residual strong force and one elementary particle that might correlate with the Yukawa potential (or, attractive component of the residual strong force). Modeling that features these two forces could parallel proposed theory modeling, based on potentials and not based on virtual gluons, for quarks in a hadron. We are uncertain as to the extent to which such modeling might provide a basis for new insight about nuclear physics. We are aware of some concern regarding modeling some aspects of nuclear physics based on the notion of virtual pions. (See reference [27].)

\subsection{Evolution of proposed theory}

This unit discusses the notion that, as of the year 2018, proposed theory seemed to achieve a stable basis of theory centric assumptions and proposed theory began to offer explanations for an increasing scope of observed natural phenomena that people have, starting in 2017 and continuing thereafter, reported.

In 2011, we decided to try to explain eras pertaining to the rate of expansion of the universe.

For years thereafter, we felt that the scope of major assumptions on which we based proposed theory grew somewhat in parallel to the scope of natural phenomena that the work seemed to explain. During this period, we did not consider the evolution of galaxies.

In 2018, the trajectories of the two scopes seemed to decouple. The scope of major assumptions seemed to stop growing. The scope of seemingly explained natural phenomena continued to grow. Newly explained natural phenomena tend to correlate with astrophysics observations that people reported during and after 2017. The astrophysical observations tend to pertain to galaxies and dark matter.

\subsection{Perspective about this essay}

This unit discusses some aspects of this essay.

We try to provide perspective regarding theories and models. Aspects of perspective include correlations with data, limits of applicability, opportunities to make improvements, unresolved aspects, and alternatives. Reference [28] suggests standards regarding such perspective.

We try to structure this essay to facilitate learning. We use an approach that blends known data, ongoing theory, development of new theory, and mathematics. We sometimes show results before we discuss theory that outputs the results.

Each one of some topics that we discuss pertains to more than one unit in this essay. We try to present units that are adequately complete. Doing so leads to double coverage regarding some aspects of some topics.

We try to accommodate readers whose familiarity with the branch of mathematics known as group theory may not be adequate for following details in some parts of this essay. The following remarks pertain. This essay uses words such as symmetries, groups, and generators. Often, relevant discussion leads to results that are integers. Some of the integers are numbers of generators (of some groups). The importance of these integers can correlate with the numbers and not necessarily with details about the relevant groups or about group theory. People do not necessarily need to understand group theory to use the integers. Regarding relevant physics modeling, the following statements pertain. Some of the integers represent so-called degrees of freedom. Some of the integers correlate with numbers of isomers. Here, use of the word isomer has parallels to uses, in chemistry, of the word isomer. 


\section{Elementary particles, field theories, and interaction vertices}

This unit lists all known elementary particles and all elementary particles that proposed theory suggests. This unit discusses properties that known elementary particles exhibit and that suggested elementary particles might exhibit. This unit discusses proposed theory complements and alternatives to ongoing theory quantum field theories.

\subsection{A table of elementary particles (or, simple particles and long-range forces)}

This unit shows a table of elementary particles that proposed theory suggests.

Table 1 catalogs elementary particles that proposed theory suggests. (Work to develop table 1 starts with discussion related to table 1 and continues through discussion related to table 28.) Our use here of the two-word term elementary particles parallels use of that term in ongoing theory. Each row in the table 1a correlates with one value of spin $S$. Here, $S$ denotes spin, in units of $\hbar$. The definition $\Sigma=2 S$ provides for numbers $\Sigma$ that are non-negative integers. The value of $\Sigma$ appears as the first element of each two-element symbol $\Sigma \Phi$. The letter value of $\Phi$ denotes a so-called family of elementary particles. The symbol $\Sigma \Phi$ denotes a so-called subfamily of elementary particles. For $\sigma=-1$, the particles model as if they occur in confined environments. Examples of confined environments include hadrons and atomic nuclei. For $\sigma=+1$, the particles model as if they can occur in confined environments and can occur outside of confined environments. We use the two-word term free environment to contrast with the twoword term confined environment. The expression $m=0$ denotes a notion of zerolike mass. Some ongoing theory models do or might correlate $m \doteq 0$ elementary fermions with small positive masses. Some ongoing theory models do or might correlate $m \doteq 0$ elementary fermions with zero masses. The expression $m>0$ correlates with mass being positive in all ongoing and proposed models. A number (n) denotes a number of elementary particles. A number $((\mathrm{n}))$ denotes a number of modes. The items 10G, 12G, ..., and 20G might provide for only negligible effects or might have no relevance to physics. (See discussion related to equations (121) and (122).) Discussion related to equations (121) and (122) suggests (but does not necessarily require) that items $10 \mathrm{G}, 12 \mathrm{G}, \ldots$, and $20 \mathrm{G}$ are not relevant to aspects of physics that correlate directly with table 1 . Nature might include 0I elementary particles. The 0I solution might correlate with ongoing theory notions of an inflaton elementary particle or of an inflaton field. (See discussion related to table 2 and discussion related to table 37.) The 0I solution might also correlate with useful aspects of mathematics-based modeling. (See discussion related to table 37b.) Table 1b provides additional information regarding items that table 1a lists.

We use the two-word term simple particle to pertain to each entry in table 1 other than G-family entries. We correlate the two-element term long-range force with each G-family entry in table 1. Modeling for each one of some long-range forces points to components for that force. Particle counts in table 1 de-emphasize modeling that would count, for example, a down quark with green color charge as differing from a down quark with red color charge.

We continue to use the two-word term simple particle. We continue to use the two-element term long-range force. We use the four-word phrase elementary particle Standard Model. Otherwise, we tend to de-emphasize the two-word term elementary particle.

We discuss the simple particles for which $\sigma=+1$ and $m>0$. The $0 \mathrm{H}$ particle is the Higgs boson. The three $1 \mathrm{C}$ particles are the three charged leptons - the electron, the muon, and the tauon. The two $2 \mathrm{~W}$ particles are the two weak interaction bosons - the $\mathrm{Z}$ boson and the $\mathrm{W}$ boson.

We discuss the simple particles for which $\sigma=+1$ and $m \doteq 0$. The $0 \mathrm{I}$, or so-called aye, particle is a possible zerolike-mass relative of the Higgs boson. The three $1 \mathrm{~N}$ particles are the three neutrinos. Some aspects of ongoing theory suggest that at least one neutrino mass must be positive. At least one positive mass might explain neutrino oscillations and some astrophysics data. Some aspects of ongoing theory, such as some aspects of the Standard Model, suggest that all neutrino masses are zero. Proposed theory suggests the possibility that neutrinos have zero mass. Proposed theory suggests that effects of $8 \mathrm{G}$ long-range forces might explain neutrino oscillations and the relevant astrophysics data.

We discuss the long-range forces. The expressions $\sigma=+1$ and $m \doteq 0$ pertain. Each G-family force exhibits two modes. Our discussion tends to focus on circularly polarized modes. One mode correlates with left circular polarization. One mode correlates with right circular polarization. For 2G, ongoing theory suggests classical physics models and quantum physics models. The word electromagnetism can pertain. Proposed theory suggests modeling that provides for $2 \mathrm{G}$ aspects that include and complement ongoing theory electromagnetism. For 4G, ongoing theory suggests classical physics models. The word gravitation can pertain. Proposed theory suggests modeling for $4 \mathrm{G}$ aspects that include and complement ongoing theory gravitation. Proposed theory regarding $4 \mathrm{G}$ includes classical physics aspects and quantum 
Table 1: Elementary particles (or, simple particles and long-range forces)

(a) Simple particles and long-range forces (with notation featuring names of families)

\begin{tabular}{|c|c|c|c|c|c|}
\hline Spin & $\Sigma$ & $\begin{array}{c}\sigma=+1 \\
m>0\end{array}$ & $\begin{array}{c}\sigma=+1 \\
m \doteq 0\end{array}$ & $\begin{array}{c}\sigma=-1 \\
m>0\end{array}$ & $\begin{array}{c}\sigma=-1 \\
m \doteq 0\end{array}$ \\
\hline 0 & 0 & $0 \mathrm{H}(1)$ & 0I (1) & $0 \mathrm{P}(1)$ & 0K (1) \\
\hline $1 / 2$ & 1 & $1 \mathrm{C}(3)$ & $1 \mathrm{~N}(3)$ & $1 \mathrm{Q}(6)$ & $1 \mathrm{R}(6)$ \\
\hline 1 & 2 & $2 \mathrm{~W}(2)$ & $2 \mathrm{G}((2))$ & $2 \mathrm{~T}(2)$ & $2 \mathrm{U}(8)$ \\
\hline 2 & 4 & & $4 \mathrm{G}((2))$ & & \\
\hline 3 & 6 & & $6 \mathrm{G}((2))$ & & \\
\hline 4 & 8 & & $8 \mathrm{G}((2))$ & & \\
\hline$\ldots$ & $\ldots$ & & & & \\
\hline 10 & 20 & & $20 \mathrm{G}((2))$ & & \\
\hline
\end{tabular}

(b) Simple particles and long-range forces (with notation featuring names; with * denoting that people might have yet to find the simple particles or long-range forces; and, with TBD denoting the three-word phrase to be determined)

\begin{tabular}{|c|c|c|c|c|c|}
\hline Spin & $\Sigma$ & $\begin{array}{c}\sigma=+1 \\
m>0\end{array}$ & $\begin{array}{c}\sigma=+1 \\
m \doteq 0\end{array}$ & $\begin{array}{c}\sigma=-1 \\
m>0\end{array}$ & $\begin{array}{c}\sigma=-1 \\
m \doteq 0\end{array}$ \\
\hline 0 & 0 & Higgs boson (1) & Aye* (1) & Pie* $^{*}(1)$ & Cake* (1) \\
\hline $1 / 2$ & 1 & Charged leptons (3) & Neutrinos (3) & Quarks (6) & Arcs* (6) \\
\hline 1 & 2 & $\mathrm{Z}$ and $\mathrm{W}$ bosons (2) & Photon $((2))$ & Tweaks* (2) & Gluons (8) \\
\hline 2 & 4 & & Graviton* $\left.{ }^{*}(2)\right)$ & & \\
\hline 3 & 6 & & $\mathrm{TBD}^{*}((2))$ & & \\
\hline 4 & 8 & & $\mathrm{TBD}^{*}((2))$ & & \\
\hline 10 & 20 & & $\mathrm{TBD}^{*}((2))$ & & \\
\hline
\end{tabular}

physics aspects. Proposed theory regarding 4G includes dark energy forces. In proposed theory, quantum interactions, involving simple fermions, mediated by gravitational aspects of $4 \mathrm{G}$ can correlate with fermion generation. In proposed theory, classical interactions with multicomponent objects can scale with the rest energy of the objects. Ongoing theory does not include 6G aspects and does not include $8 \mathrm{G}$ aspects. Proposed theory suggests that $8 \mathrm{G}$ may interact with lepton number minus baryon number. Regarding long-range forces, a lack of use of the two-word term simple particles dovetails with modeling that suggests, in some sense, more than one component for each one of some $\Sigma \mathrm{G}$.

We discuss the simple particles for which $\sigma=-1$ and $m>0$. The $0 \mathrm{P}$, or so-called pie, possible particle might correlate with an attractive component of the residual strong force. (See discussion related to equation (108).) The $0 \mathrm{P}$ particle might provide an aspect for alternative modeling regarding interactions between hadrons in atomic nuclei. The six 1Q particles are the six quarks. The two 2T, or so-called tweak, possible particles are analogs to the weak interaction bosons. The charge of the one non-zero-charge $2 \mathrm{~T}$ particle is one-third the charge of the $\mathrm{W}$ boson. The non-zero-charge tweak particle may have played a role in the creation of baryon asymmetry.

We discuss the simple particles for which $\sigma=-1$ and $m \doteq 0$. The $0 \mathrm{~K}$, or so-called cake, possible particle might correlate with a repulsive component of the residual strong force. (See discussion related to equation (108).) The $0 \mathrm{~K}$ particle might provide an aspect for alternative modeling regarding interactions between hadrons in atomic nuclei. The six 1R, or so-called arc, possible particles are zero-charge zerolike-mass analogs of the six quarks. Hadron-like particles made from arcs and gluons contain no charged particles and measure as dark matter. The eight $2 \mathrm{U}$ particles are the eight gluons.

Table 2 summarizes some possible correlations between observed phenomena, ongoing theory, and proposed theory. For each row in the table, proposed theory suggests that the item in the third column might explain aspects correlating with the other two columns. 
Table 2: Some possible correlations between observed phenomena, ongoing theory, and proposed theory

\begin{tabular}{ccc}
\hline Phenomenon & Ongoing theory & Proposed theory \\
\hline & Quantum vacuum & Aye \\
Inflaton & Aye \\
Neutrino oscillations & At least one non-zero rest mass & 8G long-range forces \\
Nuclear physics & Attractive residual strong force & Pie \\
Baryon asymmetry & & Charged tweak \\
Nuclear physics & Repulsive residual strong force & Cake \\
\hline
\end{tabular}

Table 3: Excitations for the left circular polarization mode of a photon

\begin{tabular}{ccccccccc}
\hline Side & 0 & 1,2 & 3,4 & 5,6 & 7,8 & 9,10 & 11,12 & 13,14 \\
\hline TA & $n$ & & & & & & & \\
SA & -1 & $n, @_{0}$ & & & & & & \\
\hline
\end{tabular}

\subsection{Theory that develops aspects of the table of simple particles and long- range forces}

This unit shows aspects of the framework we develop for physics theories. This unit develops aspects of the table of simple particles and long-range forces.

Ongoing theory describes photon states via two harmonic oscillators. Ongoing theory features four space-time dimensions. Why not describe photon states via four harmonic oscillators?

Proposed theory describes photon states via four harmonic oscillators. A first hunch might be that doing so correlates with non-zero longitudinal polarization and a photon rest mass that would be nonzero. However, mathematics allows a way to avoid this perceived possible problem. A second hunch might be that using four oscillators adds no insight. However, using four oscillators leads to a framework for physics theories and, eventually, even to insight about a family of phenomena that includes photons.

We consider the left circular polarization mode of a photon. We denote the number of excitations of the mode by $n$. Here, $n$ is a nonnegative integer. One temporal oscillator pertains. We label that oscillator TA0. The excitation number $n_{T A 0}=n$ pertains. Harmonic oscillator mathematics correlates a value of $n+1 / 2$ with that oscillator. Three spatial oscillators pertain. Here, $n_{S A 0}=-1, n_{S A 1}=n, n_{S A 2}=@_{0}$. Oscillator SA0 correlates with longitudinal polarization and has zero amplitude for excitation. (See equation (3).) Oscillator SA1 correlates with left circular polarization. Oscillator SA2 correlates with right circular polarization. The symbol @ denotes a value of _ that, within a context, never changes. For left circular polarization, @ $@_{0}$ pertains for oscillator SA2. The sum $n+1 / 2$ correlates with each of the one TA-side oscillator and the three SA-side oscillators. For the SA-side oscillators, the sum equals $(-1+1 / 2)+(n+1 / 2)+(0+1 / 2)$.

Table 3 shows excitations for the left circular polarization mode of a photon. For the right circular polarization mode, one exchanges the values of $n_{S A 1}$ and $n_{S A 2}$. The result is $n_{S A 1}=@_{0}, n_{S A 2}=n$.

The representation that table 3 shows is invariant with respect to observer. In ongoing theory, each observer would measure both left circular polarization and the same value of $n$. Observers might disagree with respect to measured values of energy or momentum.

The following concepts and generalizations pertain.

- The above discussion correlates with the two-word term ALG modeling. ALG is an abbreviation for the word algebraic. Later we discuss PDE modeling. PDE abbreviates the three-word term partial differential equation.

- For ALG modeling, equation (1) pertains. Each of $A_{T A}^{A L G}$ and $A_{S A}^{A L G}$ correlates with the concept of an isotropic quantum harmonic oscillator. The word isotropic (or, the two-word term equally weighted) also pertains to the pair consisting of $A_{T A}^{A L G}$ and $A_{S A}^{A L G}$. The one-element term double-entry pertains. For example, increasing a TA-side excitation number by one requires either decreasing a different TA-side excitation by one or increasing one SA-side excitation by one. The two-element term double-entry bookkeeping pertains.

$$
0=A^{A L G}=A_{T A}^{A L G}-A_{S A}^{A L G}
$$

- The above discussion extends the domain correlating with equation (2) from $n \geq 0$ to $n \geq-1$. Here, $a^{+}$denotes a harmonic oscillator raising operator. Proposed theory includes equation (3). 
Equations (4) and (5) pertain regarding a lowering operator.

$$
\begin{gathered}
a^{+}\left|n>=(1+n)^{1 / 2}\right| n+1> \\
a^{+}|-1>=0| 0> \\
a^{-}\left|n>=n^{1 / 2}\right| n-1> \\
a^{-}|0>=0|-1>
\end{gathered}
$$

- We posit that equations (6) and (7) extend equation (1). Here, the applicable number, $n$, of excitations satisfies $n \geq 0$.

$$
\begin{aligned}
& a^{+} A_{T A}^{A L G}=a^{+} A_{S A}^{A L G} \\
& a^{-} A_{T A}^{A L G}=a^{-} A_{S A}^{A L G}
\end{aligned}
$$

- One can sum any positive number of values of $A^{A L G}$. The sum is always zero. We suggest that the expression $A^{A L G}=0$ provides a basis for avoiding ongoing theory concerns about unlimited sums of ground state energies.

- Some aspects of ALG modeling include notions that people might consider to correlate with the three-word term below ground state. For example, consider the SA-side representation for the ground state of the left circular polarization mode. The proposed theory ground state sum is onehalf. People might think that the ground state sum for a three-dimensional isotropic quantum harmonic oscillator should be three-halves, as in $3 \cdot(0+1 / 2)$.

We discuss symmetries that correlate with mathematics for isotropic harmonic oscillators.

Table 4 shows groups to which proposed theory refers and shows representations that correlate with those groups. Aside from the appearance of items using the aspect $n=-1$, information in the table comports with standard relationships between mathematics of group theory and mathematics for isotropic quantum harmonic oscillators. The leftmost column shows the relevant number of oscillators. For each row except the last row, the symbol $X A$ can be TA, in which case all of the oscillators are TA-side oscillators, or SA, in which case all of the oscillators are SA-side oscillators. The symbol $S 1 G$ denotes a group with one generator. The number of generators for $U(1)$ is two. The number of generators for $S U(j)$ is $j^{2}-1$. The symbol $\pi$ correlates with the concept of permutations. The symbol $\pi_{a, b}$ denotes two possibilities. Regarding the two oscillators, for one possibility, $a$ pertains to the first oscillator and $b$ pertains to the second oscillator. For the other possibility, $a$ pertains to the second oscillator and $b$ pertains to the first oscillator. The symbol $\chi$ correlates with the concept of choice. The symbol $\chi_{(0,0),(-1,-1)}$ denotes two choices. For one choice $n_{T A 0}=n_{S A 0}=0$. For the other choice $n_{T A 0}=n_{S A 0}=-1$. The symbol $\chi_{a}$ pertains to one oscillator and correlates with the equation $n_{X A}=a$. The symbol $\kappa$ correlates with the concept of a continuous set of choices. For example, regarding two oscillators XA1 and XA2, equations (8) and (9) describe the continuum of possibilities correlating with $\kappa_{0,-1}$. Here, each of $d$ and $e$ is a complex number. Regarding $S U(j)$, each of the symbols $\kappa_{-1, \cdots,-1}$ and $\kappa_{0, \cdots, 0}$ correlates with a continuous set of choices involving amplitudes pertaining to $j$ oscillators. Equation (10) pertains regarding the symbol $\kappa_{0,0}^{\prime}$. The symbol A0- denotes $\pi_{@_{-1} @_{-1}}$. The symbol A0+ denotes $\pi_{@_{0}} @_{0}$. The symbol $\widehat{A}_{X A}^{A L G}$ denotes the contribution that the relevant oscillators make toward a total $A_{X A}^{A L G}$. The symbol $\widehat{A}_{(T A 0, S A 0)}^{A L G}$ denotes the contribution that the TA0-and-SA0 oscillator pair makes toward a total $A^{A L G}$. The symbol [blank] - in the first row of table 4 - denotes the concept that, in tables such as table 3 , one can interpret a blank cell as correlating with $\kappa_{0,-1}$.

$$
\begin{gathered}
d\left|n_{X A 1}=0, n_{X A 2}=-1>+e\right| n_{X A 1}=-1, n_{X A 2}=0> \\
|d|^{2}+|e|^{2}=1 \\
\kappa_{0,0}^{\prime}=\kappa_{0,0} \times \pi_{0,-1}
\end{gathered}
$$


Table 4: Groups and representations

\begin{tabular}{|c|c|c|c|c|c|c|c|}
\hline \multirow[t]{2}{*}{$\mathrm{O}$} & \multirow[t]{2}{*}{ Groups } & \multicolumn{2}{|c|}{$\widehat{A}_{X A}^{A L G}<0$} & \multicolumn{2}{|c|}{$A^{A L G}=0$} & \multicolumn{2}{|c|}{$\widehat{A_{X A}^{A L G}>0}$} \\
\hline & & Symbol & $\widehat{A_{X A}^{A L G}}$ & Symbols & $A^{A L G}$ & Symbol & $\widehat{A_{X A}^{A L G}}$ \\
\hline 2 & - & $\mathrm{A} 0-$ & -1 & [blank], $\kappa_{0,-1}$ & $\widehat{A}_{X A}^{A L G}=0$ & $\mathrm{~A} 0+$ & 1 \\
\hline 1 & $S 1 G$ & $\chi_{-1}$ & $-1 / 2$ & - & - & $\chi_{0}$ & $1 / 2$ \\
\hline 2 & $U(1)$ & - & - & $\pi_{0,-1}$ & $\widehat{A}_{X A}^{A L G}=0$ & $\pi_{0, @_{0}}$ & 1 \\
\hline 2 & $U(1)$ & - & - & $\pi_{@_{0}, @_{-1}}$ & $\widehat{A}_{X A}^{A L G}=0$ & - & - \\
\hline$j$ & $S U(j), j \geq 2$ & $\kappa_{-1, \cdots,-1}$ & $-j / 2$ & - & - & $\kappa_{0, \cdots, 0}$ & $j / 2$ \\
\hline 2 & $S U(2) \times U(1)$ & - & - & - & - & $\kappa_{0,0}^{\prime}$ & 1 \\
\hline 2 & $U(1)$ & - & - & $\chi_{(0,0),(-1,-1)}$ & $\widehat{A}_{(T A 0, S A 0)}^{A L G}=0$ & - & - \\
\hline
\end{tabular}

Table 5: Excitations for a photon

\begin{tabular}{ccccccccc}
\hline Side & 0 & 1,2 & 3,4 & 5,6 & 7,8 & 9,10 & 11,12 & 13,14 \\
\hline TA & $n$ & & & & & & & \\
SA & -1 & $\pi_{n, @_{0}}$ & & & & & & \\
\hline
\end{tabular}

We discuss relationships between the numbers of generators for some $S U(j)$ groups.

In equation (11), $g_{j}$ denotes the number of generators of the group $S U(j)$, the symbol $\mid$ denotes the word divides (or, the two-word phrase divides evenly), and the symbol $\mathcal{X}$ denotes the four-word phrase does not divide evenly. For some aspects of physics modeling, equation (11) correlates with ending the series $S U(3), S U(5), \cdots$ at the item $S U(7)$. For some aspects of physics modeling, the series $S U(3)$, $S U(5), S U(7)$, and $S U(17)$ might pertain.

$$
\left.\left.g_{3}\left|g_{5}, g_{3}\right| g_{7}, g_{5} \mid g_{7}, \quad g_{5}\right\} g_{9}, g_{7} X g_{9}, g_{7}\right)\left(g_{11}, \quad g_{3}\left|g_{17}, g_{5}\right| g_{17}, g_{7} \mid g_{17}\right.
$$

We anticipate invoking the mathematical notion of ending a series $S U(3), S U(5), \cdots$ at the item $S U(7)$. Sometimes, we correlate an ending with physics data. Sometimes, we correlate an ending with symmetries related to kinematics conservation laws.

We return to discussion that relates to and extends table 3.

Table 5 shows excitations for a photon. For each mode, we posit that the $U(1)$ symmetry that correlates with the permutation (appropriate to the mode) of $\pi_{n, @_{0}}$ correlates with the $U(1)$ symmetry that the elementary particle Standard Model associates with photons. One generator correlates with excitation. One generator correlates with de-excitation.

We attempt to represent elementary particles other than photons.

For this discussion, we de-emphasize addressing the following questions. To what extent do answers to the following questions differ between simple bosons and simple fermions? To what extent does $n_{S A 0}=-1$ correlate with zero longitudinal polarization? To what extent does $n_{S A 0}=-1$ correlate with zero rest mass? To what extent does $n_{S A 0}=-1$ correlate with being able to excite a state via using an arbitrarily small amount of energy squared? To what extent does $n_{S A 0}=-1$ correlate, for free environments, with travel at the speed of light? To what extent does $n_{S A 0}=-1$ correlate with inabilities to interact with phenomena, such as the Higgs boson, that modeling associates with the SA0 oscillator? To what extent does $n_{S A 0}=-1$ represent modeling correlating with circumstances that nature does not exhibit? One such circumstance might be zero entanglement between an elementary particle and the rest of the universe.

We generalize regarding work above. We assume that the oscillator pair SA1-and-SA2 correlates with charge or interactions with charge. We note that ongoing theory interrelates photons and weak interaction bosons.

Table 6 posits a ground state for weak interaction bosons. The relevant bosons are the $\mathrm{Z}$ and $\mathrm{W}$ bosons. The table correlates the negative charge state of the W boson with the SA1 oscillator. The table correlates the positive charge state of the $\mathrm{W}$ boson with the SA2 oscillator. (One might correlate negative charge with SA2 and positive charge with SA1. We do not explore this possibility further. There might be no link between this assignment regarding charge and the assignment of photon circular polarization modes.) Below, we discuss a reason, within the bounds of $A^{A L G}=0$, for placing $\kappa_{0,0}$ with the SA3-and-SA4 oscillator pair.

We discuss W-family excitations. To describe $n$ excitations of the same state of one of the bosons, we 
Table 6: Ground state for weak interaction bosons

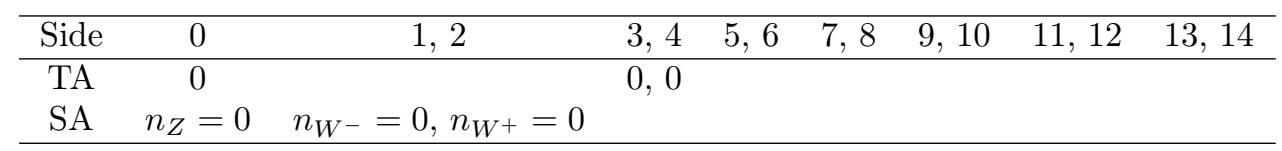

Table 7: Ground states for photons and weak interaction bosons

(a) Ground states for photons

\begin{tabular}{ccccccccc}
\hline Side & 0 & 1,2 & 3,4 & 5,6 & 7,8 & 9,10 & 11,12 & 13,14 \\
\hline TA & 0 & $\pi_{@_{0}, @_{-1}}$ & & & & & & \\
SA & -1 & $\pi_{0, @_{0}}$ & & & & & & \\
\hline
\end{tabular}

(b) Ground states for weak interaction bosons (with $\pi_{0, @_{0}, @_{0}}$ spanning the two items showing the symbol *)

\begin{tabular}{ccccccccc}
\hline Side & 0 & 1,2 & 3,4 & 5,6 & 7,8 & 9,10 & 11,12 & 13,14 \\
\hline TA & 0 & $\pi_{@_{0}, @_{-1}}$ & $\kappa_{@_{0}, @_{0}}$ & & & & & \\
SA & $*$ & $*$ & & & & & & \\
\hline
\end{tabular}

use $n_{T A 0}=n=n_{S A}$, with $\mathrm{SA}_{-}$correlating with the one boson. An isolated interaction that excites or de-excites the boson conserves the generation of the fermion that participates in the interaction. For example, an interaction between an electron (or, generation-one charged lepton) and a $\mathrm{W}^{+}$boson produces a generation-one neutrino. We say that conservation of generation pertains. We consider some interactions in hadrons (such as protons and neutrons). Here, we consider an entangled emission and absorption of a pair of $\mathrm{W}$ bosons, with one $\mathrm{W}$ boson being a $\mathrm{W}^{-}$and the other $\mathrm{W}$ boson being a $\mathrm{W}^{+}$. Ongoing theory results suggest that conservation of fermion generation need not pertain for the relevant quarks. (A transition from the state that table 6 shows to the state characterized by $n_{T A 0}=2$, $n_{S A 0}=0, n_{S A 1}=1$, and $n_{S A 2}=1$ would violate equation (6).) Equations (6) and (7) imply that at least one of oscillators TA3 and TA4 participates. Generation correlates with mass. In ongoing theory, electromagnetism correlates with spin-one long-range forces and gravitation correlates with spin two. It seems correct to associate the topic of conservation of fermion generation with oscillators TA3 and TA4. (See discussion related to equation (12).) There are three generations of quarks. Three is the number of generators of $S U(2)$. We posit that an approximate $S U(2)$ symmetry pertains. (See table 4.) We use the four-word term somewhat conservation of generation (or, the five-word term somewhat conservation of fermion generation). We note the possibility that, in appropriate settings, one might be able to detect non-conservation, induced by W-family effects, of lepton generation. Such a setting might need to be adequately conducive to multiple nearby interactions involving $\mathrm{W}$ bosons. Here, the word nearby pertains regarding both temporal aspects and spatial aspects.

We discuss an ongoing theory W-family symmetry. Ongoing theory associates $S U(2) \times U(1)$ symmetry with the weak interaction. We associate $S U(2)$ symmetry with the combination $n_{W^{-}}=0, n_{W^{+}}=0$. We associate $U(1)$ symmetry with either or both of two aspects. One aspect features the restrictions that equations (6) and (7) imply. The other aspect features the notion that, for a specific simple fermion, interactions can occur with one but not both of $\mathrm{W}^{-}$and $\mathrm{W}^{+}$. Both aspects show parallels to the $U(1)$ symmetry that pertains for photons. For the W-family, the $S U(2)$ and $U(1)$ symmetries combine to form $\kappa_{0,0}^{\prime}($ or, $S U(2) \times U(1))$.

All known interactions conserve charge. The expression $\pi_{@_{0}, @_{-1}}$ pertains and contributes $\widehat{A}_{X A}^{A L G}=0$. (See table 4.)

Table 7 retrofits conservation of charge into representations for $2 \mathrm{G}$ ground states and $2 \mathrm{~W}$ ground states.

We extend proposed theory to include the Higgs boson.

Table 8 shows excitations for the Higgs (or, $0 \mathrm{H}$ ) boson. The ground state value $n_{S A 0}=0$ correlates with the non-zero mass of the Higgs boson. The lack of an SA1-and-SA2 entry correlates with the Higgs boson having zero charge and not interacting with charge.

We note that a number of SA-side oscillators seems to correlate with each of spin and numbers of particles.

For each of $0 \mathrm{H}, 2 \mathrm{~W}$, and $2 \mathrm{G}$, equation (12) pertains. In the equation, $N_{S A}$ denotes a number of relevant SA-side oscillators. Also, for $0 \mathrm{H}$ and $2 \mathrm{~W}, 2 S+1$ provides the number of particles, if one counts 
Table 8: Excitations for the Higgs boson

\begin{tabular}{ccccccccc}
\hline Side & 0 & 1,2 & 3,4 & 5,6 & 7,8 & 9,10 & 11,12 & 13,14 \\
\hline TA & $n$ & $\pi_{@_{0}, @_{-1}}$ & & & & & & \\
SA & $n$ & & & & & & & \\
\hline
\end{tabular}

Table 9: States for 0I, 1C, and 1N simple particles

(a) Ground state for 0I bosons

\begin{tabular}{ccccccccc}
\hline Side & 0 & 1,2 & 3,4 & 5,6 & 7,8 & 9,10 & 11,12 & 13,14 \\
\hline TA & -1 & $\pi_{@_{0}, @_{-1}}$ & & & & & & \\
SA & -1 & & & & & & & \\
\hline
\end{tabular}

(b) States for charged leptons

\begin{tabular}{ccccccccc}
\hline Side & 0 & 1,2 & 3,4 & 5,6 & 7,8 & 9,10 & 11,12 & 13,14 \\
\hline TA & 0 & $\pi_{@_{0}, @_{-1}}$ & $\kappa_{@_{0}, @_{0}}$ & & & & & \\
SA & 0 & $\pi_{0,-1}$ & $\kappa_{0,0}$ & & & & & \\
\hline
\end{tabular}

(c) States for neutrinos

\begin{tabular}{ccccccccc}
\hline Side & 0 & 1,2 & 3,4 & 5,6 & 7,8 & 9,10 & 11,12 & 13,14 \\
\hline TA & -1 & $\pi_{@_{0}, @_{-1}}$ & $\kappa_{@_{-1}, @_{-1}}$ & & & & & \\
SA & -1 & $\pi_{0,-1}$ & $\kappa_{-1,-1}$ & & & & & \\
\hline
\end{tabular}

matter particles and antimatter particles separately.

$$
N_{S A}=2 S+1
$$

We extend work above to include all known and suggested simple particles and long-range forces for which $\sigma=+1$. (See table 1.)

Table 9 shows states for 0I, 1C, and 1N simple particles. Each state for which the one-word term boson (or, the result that $2 S$ is an even integer) pertains comports with equation (12). For fermions (or, for particles for which $2 S=1$ ), equation (12) pertains, given two assumptions. One assumption is that we do not count the SA3-and-SA4 oscillator pair, which correlates with three generations. One assumption is that each of $n_{S A 1}=-1$ and $n_{S A 2}=-1$ disables one oscillator and, in effect, leads to the result $N_{S A}=2$. The simple particle 0I might seem to be not capable of excitation. The 0I particle might play roles in some models. (See table 37b.) Table 37a suggests that nature might include 0I particles. For each of charged leptons and neutrinos, the states are either populated or not populated. Each of charged leptons and neutrinos exhibits a TA3-and-TA4 approximate $S U(2)$ symmetry. That symmetry suggests approximate conservation of fermion generation. Each state that table 9 shows comports, via a TA1-and-TA2 entry, with conservation of charge. For each of charged leptons and neutrinos, the SA1and-SA2 appearance of a $U(1)$ symmetry may seem surprising. Unlike for bosons, multiple excitations for a single state do not pertain. However, multicomponent objects can include more than one identical (for this discussion) fermion. For example, an atom can contain more than one electron.

We note an aspect regarding $\mathrm{W}$ bosons and leptons. (See tables 7b, 9b, and 9c.) SA1-and-SA2 oscillator pairs correlate with allowed interactions between $\mathrm{W}$ bosons and leptons. Alignment of $n_{S A}=0$ for the $\mathrm{W}$ boson with one of $n_{S A}=0$ and $n_{S A}=-1$ correlates with an allowed absorption of charge (from the $\mathrm{W}$ boson) by a matter charged lepton. Apparently, either choice between $n_{S A}=0$ and $n_{S A}=-1$ can be appropriate, as long as one treats other choices consistently with a first choice. For example, for a choice of $n_{S A}=0$ for a matter charged lepton (such as an electron), the following statements pertain. For matter charged leptons, $n_{S A}=-1$ correlates with an ability to shed a unit of charge. For antimatter charged leptons ( a unit of charge. For antimatter charged leptons, $n_{S A}=0$ correlates with an ability to shed a unit of charge. For neutrinos, parallels to the above four relationships hold to the extent that one follows the instructions that the next four sentences provide. Substitute the word neutrino for the two-word construct charged lepton. Substitute the word antimatter for the word matter. Substitute the word matter for the word antimatter. Drop the two parenthetical remarks that begin with the two-word construct such as.

Table 10 shows one possibility for states for 4G, 6G, and 8G long-range forces. Each state comports 
Table 10: One possibility for states for $4 \mathrm{G}, 6 \mathrm{G}$, and $8 \mathrm{G}$ long-range forces

(a) States for $4 \mathrm{G}$ bosons

\begin{tabular}{ccccccccc}
\hline Side & 0 & 1,2 & 3,4 & 5,6 & 7,8 & 9,10 & 11,12 & 13,14 \\
\hline TA & $n$ & $\pi_{@_{0}, @_{-1}}$ & $\kappa_{@_{0}, @_{0}}$ & & & & & \\
SA & -1 & $\pi_{@_{0}, @_{0}}$ & $\pi_{n, @_{0}}$ & & & & & \\
\hline
\end{tabular}

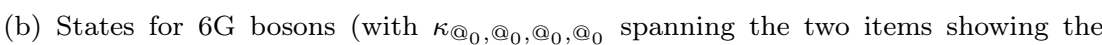
symbol *)

\begin{tabular}{ccccccccc}
\hline Side & 0 & 1,2 & 3,4 & 5,6 & 7,8 & 9,10 & 11,12 & 13,14 \\
\hline TA & $n$ & $\pi_{@_{0}, @_{-1}}$ & $*$ & $*$ & & & & \\
SA & -1 & $\pi_{@_{0}, @_{0}}$ & $\pi_{@_{0}, @_{0}}$ & $\pi_{n, @_{0}}$ & & & & \\
\hline
\end{tabular}

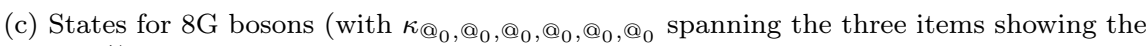
symbol *)

\begin{tabular}{ccccccccc}
\hline Side & 0 & 1,2 & 3,4 & 5,6 & 7,8 & 9,10 & 11,12 & 13,14 \\
\hline TA & $n$ & $\pi_{@_{0}, @_{-1}}$ & $*$ & $*$ & $*$ & & & \\
SA & -1 & $\pi_{@_{0}, @_{0}}$ & $\pi_{@_{0}, @_{0}}$ & $\pi_{@_{0}, @_{0}}$ & $\pi_{n, @_{0}}$ & & & \\
\hline
\end{tabular}

Table 11: Another possibility for states for 4G, 6G, and $8 \mathrm{G}$ long-range forces

(a) States for $4 \mathrm{G}$ bosons

\begin{tabular}{ccccccccc}
\hline Side & 0 & 1,2 & 3,4 & 5,6 & 7,8 & 9,10 & 11,12 & 13,14 \\
\hline TA & $n$ & $\pi_{@_{0}, @_{-1}}$ & & & & & & \\
SA & -1 & & $\pi_{n, @_{0}}$ & & & & & \\
\hline
\end{tabular}

(b) States for $6 \mathrm{G}$ bosons

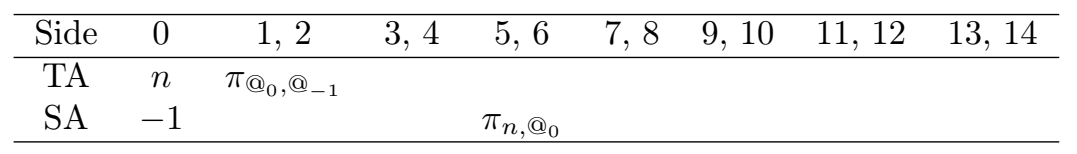

(c) States for $8 \mathrm{G}$ bosons

\begin{tabular}{ccccccccc}
\hline Side & 0 & 1,2 & 3,4 & 5,6 & 7,8 & 9,10 & 11,12 & 13,14 \\
\hline TA & $n$ & $\pi_{@_{0}, @_{-1}}$ & & & & & & \\
SA & -1 & & & & $\pi_{n, @_{0}}$ & & & \\
\hline
\end{tabular}

with equation (12). Here, $4 \mathrm{G}$ exhibits a TA3-and-TA4 approximate $S U(2)$ symmetry. That symmetry suggests approximate conservation of fermion generation. Each of $6 \mathrm{G}$ and $8 \mathrm{G}$ does not exhibit a symmetry that correlates with approximate conservation of fermion generation. For $6 \mathrm{G}$, the symmetry is $S U(4)$. For $8 \mathrm{G}$, the symmetry is $S U(6)$. Each state that table 10 shows comports, via a TA1-and-TA2 entry, with conservation of charge.

Table 11 shows another possibility for states for 4G, 6G, and 8G long-range forces. Each state does not comport with equation (12). Each state does not necessarily correlate with approximate conservation of fermion generation. Each state that table 11 shows comports, via a TA1-and-TA2 entry, with conservation of charge.

Proposed theory emphasizes table 11 and de-emphasizes table 10. The work uses aspects of table 17 in models for components of $\Sigma \mathrm{G}$ bosons. One aspect correlates with approximate conservation of fermion generation.

We extend proposed theory to include simple particles for which $\sigma=-1$ and $m>0$.

Table 12 posits information regarding simple particles for which $\sigma=-1$ and $m>0$. Table 12 a differs

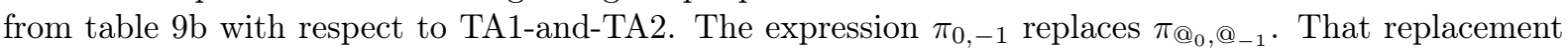
dovetails with the existence of six matter quarks (and six antimatter quarks), compared with the existence of three matter charged leptons (and three antimatter charged leptons). Interactions between quarks and $\mathrm{W}$ bosons parallel interactions between charged leptons and $\mathrm{W}$ bosons. The new TA1-and-TA2 entry correlates with conservation of charge for those interactions. People know of such interactions. Ongoing theory comports with the notion that those interactions do not convert antimatter quarks into matter 
Table 12: Simple particles for which $\sigma=-1$ and $m>0$

(a) States for quarks

\begin{tabular}{ccccccccc}
\hline Side & 0 & 1,2 & 3,4 & 5,6 & 7,8 & 9,10 & 11,12 & 13,14 \\
\hline TA & 0 & $\pi_{0,-1}$ & $\kappa_{@_{0}, @_{0}}$ & & & & & \\
SA & 0 & $\pi_{0,-1}$ & $\kappa_{0,0}$ & & & & & \\
\hline
\end{tabular}

(b) Ground states for $2 \mathrm{~T}$ bosons (with $\pi_{0, @_{0}, @_{0}}$ spanning the two items showing the symbol *)

\begin{tabular}{ccccccccc}
\hline Side & 0 & 1,2 & 3,4 & 5,6 & 7,8 & 9,10 & 11,12 & 13,14 \\
\hline TA & $*$ & $*$ & & & & & & \\
SA & 0 & $\pi_{@_{0}, @_{-1}}$ & $\kappa_{@_{0}, @_{0}}$ & & & & & \\
\hline
\end{tabular}

(c) Excitations for the 0P boson

\begin{tabular}{ccccccccc}
\hline Side & 0 & 1,2 & 3,4 & 5,6 & 7,8 & 9,10 & 11,12 & 13,14 \\
\hline TA & $n$ & $\pi_{@_{0}, @_{-1}}$ & & & & & & \\
SA & $n$ & & & & & & & \\
\hline
\end{tabular}

quarks and do not convert matter quarks into antimatter quarks. (Remarks regarding table 22 discuss adding a symmetry that correlates with conservation of lepton number minus baryon number. Remarks regarding table 22 discuss adding an approximate symmetry that correlates with somewhat conservation of baryon number.) The new TA1-and-TA2 entry allows for interactions with simple bosons that would convert antimatter quarks into matter quarks and would convert matter quarks into antimatter quarks. We posit that the magnitude of the amount of charge transferred is one-third the magnitude of the charge of the $\mathrm{W}$ boson. We posit that a charged $2 \mathrm{~T}$ boson correlates with the transfer of charge. Early in the development of the universe, charged $2 \mathrm{~T}$ bosons might have catalyzed the formation of baryon asymmetry. Table $12 \mathrm{~b}$ pertains for $2 \mathrm{~T}$ bosons. The TA-side information in table $12 \mathrm{~b}$ parallels the SAside information in table $7 \mathrm{~b}$. The SA-side information in table $12 \mathrm{~b}$ parallels the TA-side information in table 7b. Regarding quarks, W-family interactions conserve baryon number, interactions with the neutral T-family boson conserve baryon number, and interactions with the charged T-family boson do not conserve baryon number. The SA1-and-SA2 entries for quarks and the SA1-and-SA2 entry for $2 \mathrm{~T}$ bosons correlate with conservation of charge for interactions between quarks and T-family bosons. Table $12 \mathrm{c}$ pertains to $0 \mathrm{P}$ bosons. Table $12 \mathrm{c}$ mimics table 8 , which pertains for the Higgs boson. Tweak (or, 2T) bosons intermediate interactions that involve quarks. Quarks and 2T bosons can pertain inside hadronlike particles. Pie (or, 0P) bosons intermediate interactions between hadron-like particles. Hadron-like particles and $0 \mathrm{P}$ bosons can pertain inside atomic nuclei.

We extend proposed theory to include simple particles for which $\sigma=-1$ and $m \doteq 0$.

Table 13 posits information regarding simple particles for which $\sigma=-1$ and $m \doteq 0$. Table 13a differs from table $12 \mathrm{a}$ with respect to TA0 and with respect to SA0. In each case the expression -1 replaces a zero. That replacement dovetails with the notion that so-called arcs (or, 1R particles) have zero charge. Regarding gluons (or, $2 \mathrm{U}$ bosons) and cake (or, $0 \mathrm{~K}$ ) bosons, we posit that values of $n_{X A 0}=-1$ do not necessarily correlate with no ability to excite. (Regarding gluons, see discussion related to table 45 .) These bosons occur, in today's universe, in confined environments. Table $13 \mathrm{~b}$ pertains for gluons. The statement $n_{S A 0}=-1$ echoes the ongoing theory notion that gluons have zero mass. The XA1-and-XA2 entries correlate with no interaction with charge. (Here, XA can be either TA or SA.) The XA3-and-XA4 entries correlate with no interaction with generation or rest mass. The $\kappa_{-1,-1,-1}$ symmetry echoes the ongoing theory notion of $S U(3)$ symmetry for the strong interaction. (See discussion related to table 45.) The choice of TA5-and-TA6 correlates with the notion that SA5-and-SA6 correlates with baryons but not necessarily with baryon number. (Discussion related to table 21 suggests that TA7-and-TA8 and SA7-and-SA8 correlate with lepton number minus baryon number. TA5-and-TA6 pertains, by default, regarding color charge.) The SA5-and-SA6 entry correlates with a lack of conservation of color charge. Gluons (or, 2U bosons) intermediate interactions that involve quarks. Quarks and gluons can pertain inside hadron-like particles. Cake (or, 0K) bosons intermediate interactions between hadron-like particles. Hadron-like particles and 0K bosons can pertain inside atomic nuclei.

Results above leave unanswered questions such as the following. Does nature include all the simple particles and long-range forces that table 1 suggests? Does nature include simple particles and long-range forces that table 1 does not suggest? Might further modeling that pertains to $2 \mathrm{G}$ dovetail with known 
Table 13: Simple particles for which $\sigma=-1$ and $m \doteq 0$

(a) States for $1 \mathrm{R}$ fermions

\begin{tabular}{ccccccccc}
\hline Side & 0 & 1,2 & 3,4 & 5,6 & 7,8 & 9,10 & 11,12 & 13,14 \\
\hline TA & -1 & $\pi_{0,-1}$ & $\kappa_{@_{-1}, @_{-1}}$ & & & & & \\
SA & -1 & $\pi_{0,-1}$ & $\kappa_{-1,-1}$ & & & & & \\
\hline
\end{tabular}

(b) A representation regarding gluons (with $\kappa_{-1,-1,-1}$ spanning the two items showing the symbol *)

\begin{tabular}{ccccccccc}
\hline Side & 0 & 1,2 & 3,4 & 5,6 & 7,8 & 9,10 & 11,12 & 13,14 \\
\hline TA & $*$ & $\pi_{@_{0}, @_{-1}}$ & $\kappa_{@_{-1}, @_{-1}}$ & $*$ & & & & \\
SA & -1 & $\pi_{@_{0}, @_{-1}}$ & $\kappa_{@_{-1}, @_{-1}}$ & $\kappa_{@_{-1}, @_{-1}}$ & & & & \\
\hline
\end{tabular}

(c) A representation regarding the $0 \mathrm{~K}$ boson

\begin{tabular}{ccccccccc}
\hline Side & 0 & 1,2 & 3,4 & 5,6 & 7,8 & 9,10 & 11,12 & 13,14 \\
\hline TA & -1 & $\pi_{@_{0}, @_{-1}}$ & & & & & & \\
SA & -1 & & & & & & & \\
\hline
\end{tabular}

Table 14: Symmetries correlating with kinematics conservation laws

\begin{tabular}{ccc}
\hline Conservation law & Ongoing theory & Proposed theory \\
\hline Conservation of energy & $S 1 G$ & TA-side $S U(5)$ \\
Conservation of linear momentum & $S U(2)$ & SA-side $S U(2)$ \\
Conservation of angular momentum & $S U(2)$ & SA-side $S U(2)$ \\
\hline
\end{tabular}

details regarding electromagnetism? Might further modeling regarding $4 \mathrm{G}$ describe dark energy forces? To what extent does our modeling extend to include multicomponent objects? Does our modeling extend to include measured quantities such as energy and momentum?

We address such questions below.

\subsection{Kinematics conservation laws}

This unit shows modeling regarding conservation of energy, momentum, and angular momentum.

In ongoing theory, the electromagnetic field carries information that correlates with events that excited the field. Via de-excitations, people measure energies, momenta, and polarizations. People infer information about excitation events.

We want to discuss the extent to which models for $\Sigma \mathrm{G}$ (or, G-family) fields reflect encoded information.

We start by exploring modeling related to energy, momentum, and angular momentum.

Ongoing theory discusses models for objects, internal properties (such as spin and charge) of objects, motion-centric properties (such as linear momentum) of objects, and interactions (or, forces) that affect internal properties of objects or motion of objects.

We discuss symmetries that ongoing theory and proposed theory correlate with conservation laws related to motion.

Table 14 summarizes symmetries correlating with kinematics conservation laws. Ongoing theory correlates an $S 1 G$ symmetry with conservation of energy. The one-element term $S 1 G$ denotes a symmetry correlating with a group for which one generator pertains. Proposed theory considers this $S 1 G$ symmetry to be a TA-side symmetry. To some extent, proposed theory considers that this $S 1 G$ symmetry correlates with the TA0 oscillator. Ongoing theory correlates an $S U(2)$ symmetry with conservation of linear momentum and an $S U(2)$ symmetry with conservation of angular momentum. We consider each of these $S U(2)$ symmetries to be one SA-side symmetry.

The following concepts pertain.

- We extend the notion of $\sigma=+1$ to include free-ranging objects other than the $\sigma=+1$ simple particles and long-range forces to which table 1 alludes. The notion of free-ranging correlates with an object having a well-specified definition and modeling, under some circumstances, as if conservation of energy, momentum, and angular momentum pertain for the object. 
Table 15: Conservation of energy, momentum, and angular momentum for objects for which $\sigma=+1$

(a) The case $n_{T A 0}=0$ (with $\kappa_{0,0,0,0,0}$ spanning the three items showing the symbol $*$ )

\begin{tabular}{ccccccccc}
\hline Side & 0 & 1 and 2 & 3 and 4 & 5 and 6 & 7 and 8 & 9 and 10 & 11 and 12 & 13 and 14 \\
\hline TA & $*$ & & & & & & $*$ & $*$ \\
SA & 0 & & & & & & $\kappa_{0,0}$ & $\kappa_{0,0}$ \\
\hline
\end{tabular}

(b) The case $n_{T A 0}=-1$ (with $\kappa_{-1,-1,-1,-1,-1}$ spanning the three items showing the symbol *)

\begin{tabular}{ccccccccc}
\hline Side & 0 & 1 and 2 & 3 and 4 & 5 and 6 & 7 and 8 & 9 and 10 & 11 and 12 & 13 and 14 \\
\hline TA & $*$ & & & & & & $*$ & $*$ \\
SA & -1 & & & & & & $\kappa_{-1,-1}$ & $\kappa_{-1,-1}$ \\
\hline
\end{tabular}

- Models for the kinematics of objects for which $\sigma=+1$ need to include the possibility that all three conservation laws pertain. The relevance of all three conservation laws correlates with modeling that correlates with the notion of a distinguishable object and with the notion of a free environment. (Objects for which $\sigma=+1$ can exist as components of, let us call them, larger objects for which $\sigma=+1$. For one example, an electron can exist as part of an atom. For another example, a hadron can exist as part of an atomic nucleus that includes more than one hadron. In such contexts, modeling of the kinematics of the electron or hadron does not necessarily need to embrace all three conservation laws.)

- Models regarding the kinematics of objects for which $\sigma=-1$ do not necessarily need to embrace all three kinematics conservation laws. (These objects model as existing in the contexts of $\sigma=+1$ larger objects. See, for example, discussion related to table 44.)

- For an ALG model to embrace conservation of linear momentum and conservation of angular momentum, one, in effect, adds (to a model for an object) four SA-side oscillators and expresses two instances of $S U(2)$ symmetry. Double-entry bookkeeping suggests adding four TA-side oscillators. For at least some modeling, proposed theory suggests combining the four TA-side oscillators with the TA0 oscillator to correlate with an $S U(5)$ symmetry. Proposed theory suggests that, for such modeling, for each of the eight added oscillators, $n=n_{T A 0}$. For such modeling, proposed theory suggests that the TA-side $S U(5)$ symmetry correlates with conservation of energy.

- Table 15 shows representations of kinematics conservation laws for objects for which $\sigma=+1$. The choice of oscillator pairs XA11-and-XA12 and XA13-and-XA14 correlates with the possibilities for other uses for oscillators XA0-through-XA10. (See discussion related to equation (123).) Here, we know of no correlation between oscillator pair SA11-and-SA12 and spin (for example, a spin of six). Here, we know of no correlation between oscillator pair SA13-and-SA14 and spin (for example, a spin of seven).

- Special relativity correlates with boost symmetry, which is an additional $S U(2)$ symmetry. We suggest the possibility for using the oscillator pair SA15-and-SA16 to represent boost symmetry or a lack of boost symmetry. When boost symmetry applies, we suggest not extending the TAside symmetry from $S U(5)$ to $S U(7)$. Boost correlates with modeling and not with kinematics conservation laws.

- The following modeling can pertain regarding combining two $\sigma=+1$ objects to form a $\sigma=+1$ object.

- Each of the two original objects contributes two SA-side $S U(2)$ symmetries.

- The new object embraces two SA-side $S U(2)$ symmetries regarding its internal aspects. The other two original $S U(2)$ symmetries are available regarding the motion of the new object. Neither of the original two objects continues to exhibit both conservation of momentum and conservation of angular momentum. For example, for a system consisting of a star and planet, neither the star nor the planet exhibits conservation of momentum. In this context, kinematics modeling for each of the two original objects might correlate with $\sigma=-1$ modeling.

- Similarly, the new object embraces one TA-side $S U(5)$ symmetry regarding its internal aspects. Another $S U(5)$ symmetry is available regarding the motion of the new object. 
- The following modeling can pertain regarding combining $\sigma=-1$ simple fermions and $\sigma=-1$ simple bosons to form a $\sigma=+1$ object.

- For modeling the internals of a hadron (such as a proton), two possibilities exist.

1. One can model each of individual quarks and individual gluons as correlating with conservation of energy, momentum, and angular momentum. Ongoing theory quantum chromodynamics correlates with this possibility. Modeling includes notions of virtual particles.

2. One can model each of quarks and the strong interaction as correlating with just one SAside $S U(2)$ symmetry. (To the extent that modeling for the hadron correlates with boost symmetry, the strong interaction contributes the needed third SA-side $S U(2)$ symmetry.) Modeling that features quarks and potentials pertains. Modeling does not necessarily feature virtual particles.

- The 24-fold symmetry that TA-side $S U(5)$ symmetry suggests might correlate with aspects of simple particles and long-range forces. (Regarding the symmetry, see table 14. Regarding aspects of simple particles and long-range forces, see discussion related to equation (103).)

\subsection{Long-range forces, including interactions with lepton number minus baryon number}

This unit discusses aspects regarding long-range forces. This unit shows modeling that links free-ranging simple bosons and long-range forces. This unit discusses modeling pertaining to anomalous moments, including anomalous magnetic dipole moments. This unit suggests that some long-range forces interact with lepton number minus baryon number. This unit suggests alternatives to the ongoing theory notions that observations about neutrinos imply that at least one generation of neutrino has non-zero mass.

We explore modeling that encodes, regarding $2 \mathrm{G}$ modes, information about excitations of the overall $2 \mathrm{G}$ field. We consider the left circular polarized mode. Modeling for some excitations correlates with aspects of table 3 . We might also consider an excitation that models as combining an excitation of the left circular mode of $4 \mathrm{G}$ and the right circular mode of $2 \mathrm{G}$. The combination yields a left circular polarization spin-1 excitation.

Equation (13) provides notation that we use for such combinations. The symbol $\Sigma G$ denotes a subfamily of the G-family of solutions to equation (1). The symbol $\Gamma$ denotes a set of even integers selected from the set $\{2,4,6,8\}$. We use the symbol $\lambda$ to denote an element of $\Gamma$. The value $j$ correlates with the oscillator pair $\mathrm{SA}(j-1)$-and-SA $j$. (Later, we discuss aspects correlating with the limit $\lambda \leq 8$. For example, see discussion, related to equation (48), about channels. For example, see discussion related to table 17.) For the above example of subtracting spin- 1 from spin-2, the notation $\Gamma=24$ pertains and equation (14) pertains.

$$
\begin{gathered}
\Sigma \mathrm{G} \Gamma \\
\Sigma=|-2+4|
\end{gathered}
$$

Table 16 points to possibly relevant solutions. The label monopole correlates with one solution per item. The label dipole correlates with two solutions per item. The label quadrupole correlates with four mathematical solutions per item. G-family physics does not include phenomena that might correlate with the symbol 0G. For each of two quadrupole items, the one 0GГ mathematical solution is not relevant to G-family physics. For example, the solution 0G246, which correlates with $|-2-4+6|$, is not relevant to G-family physics. The label octupole correlates with eight mathematical solutions per item. The solution 0G2468 is not relevant to G-family physics. The table notes a conceptually possible 0G $\emptyset$ solution. The symbol $\emptyset$ denotes the empty set.

Above, the terms monopole through octupole correlate with numbers of solutions and not necessarily with physical phenomena.

So far, proposed theory does not depend on choosing a kinematics model. Examples of kinematics models include Newtonian physics and general relativity.

We posit that the words monopole through octupole correlate, for Newtonian physics modeling, with force laws. Ongoing theory correlates the word monopole with a potential energy that varies as $r^{-1}$ and with the RSDF of $r^{-2}$. Here, $r$ denotes the distance from the center of the one relevant object. RSDF abbreviates the five-word term radial spatial dependence of force. Here, we de-emphasize angular aspects of forces. 
Table 16: G-family solutions that may be relevant

\begin{tabular}{ccccc}
\hline Other & Monopole & Dipole & Quadrupole & Octupole \\
\hline 0G $\emptyset$ & $2 \mathrm{G} 2$ & $\Sigma \mathrm{G} 24$ & $\Sigma \mathrm{G} 246$ & $\Sigma \mathrm{G} 2468$ \\
& $4 \mathrm{G} 4$ & $\Sigma \mathrm{G} 26$ & $\Sigma \mathrm{G} 248$ & \\
& $6 \mathrm{G} 6$ & $\Sigma \mathrm{G} 28$ & $\Sigma \mathrm{G} 268$ & \\
& $8 \mathrm{G} 8$ & $\Sigma \mathrm{G} 46$ & $\Sigma \mathrm{G} 468$ & \\
& & $\Sigma \mathrm{G} 48$ & & \\
& & $\Sigma \mathrm{G} 68$ & & \\
\hline
\end{tabular}

Table 17: Information, including TA-side symmetries, regarding G-family solutions

\begin{tabular}{|c|c|c|c|c|c|c|c|c|}
\hline$\Sigma \Phi \Gamma$ & $\begin{array}{c}\text { Span } \\
\text { (for } \\
n \geq 6 \text { ) }\end{array}$ & $\begin{array}{c}\text { TA-side } \\
S U\left(\_\right) \\
\text {symmetry }\end{array}$ & $\begin{array}{c}\mathrm{TA} \\
0\end{array}$ & $\begin{array}{c}\mathrm{SA} \\
0\end{array}$ & $\begin{array}{c}\text { SA } \\
1 \text { and } 2\end{array}$ & $\begin{array}{c}\text { SA } \\
3 \text { and } 4\end{array}$ & $\begin{array}{c}\text { SA } \\
5 \text { and } 6\end{array}$ & $\begin{array}{c}\mathrm{SA} \\
7 \text { and } 8\end{array}$ \\
\hline $0 \mathrm{G} \emptyset$ & 1 & None & -1 & -1 & & & & \\
\hline $2 \mathrm{G} 2$ & 1 & None & 0 & -1 & $\pi_{0, @_{0}}$ & & & \\
\hline $4 \mathrm{G} 4$ & 6 & $S U(3)$ & 0 & -1 & $\mathrm{~A} 0+$ & $\pi_{0, @_{0}}$ & & \\
\hline$\Sigma \mathrm{G} 24$ & 1 & None & 0 & -2 & $\pi_{0, @_{0}}$ & $\pi_{0, @_{0}}$ & & \\
\hline 6G6 & 2 & $S U(5)$ & 0 & -1 & $\mathrm{~A} 0+$ & $\mathrm{A} 0+$ & $\pi_{0, @_{0}}$ & \\
\hline$\Sigma \mathrm{G} 26$ & 6 & $S U(3)$ & 0 & -2 & $\pi_{0, @_{0}}$ & $\mathrm{~A} 0+$ & $\pi_{0, @_{0}}$ & \\
\hline$\Sigma \mathrm{G} 46$ & 6 & $S U(3)$ & 0 & -2 & $\mathrm{~A} 0+$ & $\pi_{0, @_{0}}$ & $\pi_{0, @_{0}}$ & \\
\hline$\Sigma \mathrm{G} 246$ & 1 & None & 0 & -3 & $\pi_{0, @_{0}}$ & $\pi_{0, @_{0}}$ & $\pi_{0, @_{0}}$ & \\
\hline 8G8 & 1 & $S U(7)$ & 0 & -1 & $\mathrm{~A} 0+$ & $\mathrm{A} 0+$ & $\mathrm{A} 0+$ & $\pi_{0, @_{0}}$ \\
\hline$\Sigma G 28$ & 2 & $S U(5)$ & 0 & -2 & $\pi_{0, @_{0}}$ & $\mathrm{~A} 0+$ & $\mathrm{A} 0+$ & $\pi_{0, @_{0}}$ \\
\hline$\Sigma \mathrm{G} 48$ & 2 & $S U(5)$ & 0 & -2 & $\mathrm{~A} 0+$ & $\pi_{0, @_{0}}$ & $\mathrm{~A} 0+$ & $\pi_{0, @_{0}}$ \\
\hline$\Sigma G 68$ & 2 & $S U(5)$ & 0 & -2 & $\mathrm{~A} 0+$ & $\mathrm{A} 0+$ & $\pi_{0, @_{0}}$ & $\pi_{0, @_{0}}$ \\
\hline$\Sigma G 248$ & 6 & $S U(3)$ & 0 & -3 & $\pi_{0, @_{0}}$ & $\pi_{0, @_{0}}$ & $\mathrm{~A} 0+$ & $\pi_{0, @_{0}}$ \\
\hline$\Sigma \mathrm{G} 268$ & 6 & $S U(3)$ & 0 & -3 & $\pi_{0, @_{0}}$ & $\mathrm{~A} 0+$ & $\pi_{0, @_{0}}$ & $\pi_{0, @_{0}}$ \\
\hline$\Sigma G 468$ & 6 & $S U(3)$ & 0 & -3 & $\mathrm{~A} 0+$ & $\pi_{0, @_{0}}$ & $\pi_{0, @_{0}}$ & $\pi_{0, @_{0}}$ \\
\hline$\Sigma \mathrm{G} 2468$ & 1 & None & 0 & -4 & $\pi_{0, @_{0}}$ & $\pi_{0, @_{0}}$ & $\pi_{0, @_{0}}$ & $\pi_{0, @_{0}}$ \\
\hline
\end{tabular}

Table 17 shows representations for the G-family solutions that table 16 lists. The solutions correlate with symmetries pertaining to ground states. Excitations for a $\Sigma$ G $\Gamma$ solution comport with the type of $\Sigma$ G excitations to which table 11 alludes for the cases of $\Sigma$ being four, six, or eight. In table 17. the rightmost seven columns comport with double-entry bookkeeping. For example, a TA-side $S U(3)$ symmetry alludes to two additional TA-side oscillators for each of which $n_{T A}=0$. Those two oscillators plus the TA0 oscillator correlate with $\kappa_{0,0,0}$ (or, with $S U(3)$ ). The symbol $\mathrm{A} 0+$ correlates with an oscillator pair for which, for each of the two oscillators, the symbol $@_{0}$ pertains. The column regarding span pertains regarding aspects of dark matter. (See table 40.) Regarding each $\Sigma>0$ solution that the table shows, the radial behavior of the potential is $r^{n_{S A 0}}$. The RSDF is $r^{n_{S A 0}-1}$.

Regarding elementary particle physics, we posit that the series $2 \mathrm{G} 2,4 \mathrm{G} 4, \ldots$ ends with $8 \mathrm{G} 8$. $\Gamma$.

We note two notions that might correlate with that limit. Each notion correlates with components of

The limit might correlate with a scaling law. For the $\Gamma$ of $2468 \llbracket 10 \rrbracket$, the one-element phrase hexadecimal-

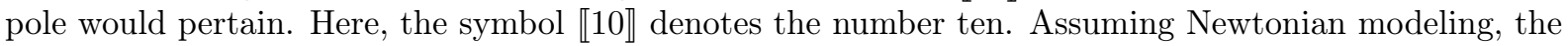
RSDF (or, radial spatial dependence of force) would be $r^{-6}$. We consider two similar, neighboring, non-

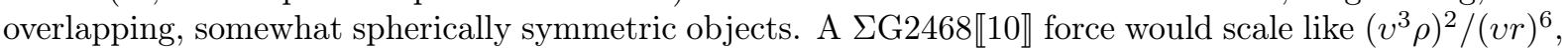
in which $v$ is a non-dimensional scaling factor that correlates with linear size (or, a length), $\rho$ is the relevant object property for the case for which $v=1$, and $r$ is the distance between the centers of the objects. The factor $v^{3}$ provides for scaling for an object that has three spatial dimensions. The force would be independent of $v$. That independence might suggest, from a standpoint of physics, that $\varrho=0$ pertains.

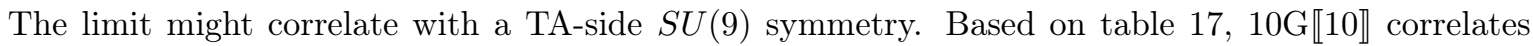

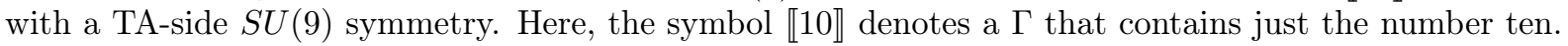

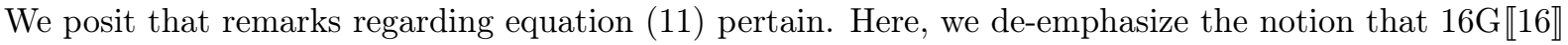

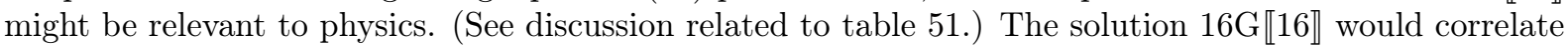
with TA-side $S U(17)$ symmetry. 
Table 18: $\Sigma \gamma$ solutions (or, G-family solutions for which $\Sigma$ appears in the list $\Gamma$ )

\begin{tabular}{ccccc}
\hline$\Sigma$ & Monopole & Dipole & Quadrupole & Octupole \\
\hline 2 & $2 \mathrm{G} 2$ & $2 \mathrm{G} 24$ & $2 \mathrm{G} 248$ & \\
4 & $4 \mathrm{G} 4$ & $4 \mathrm{G} 48$ & $4 \mathrm{G} 246$ & $4 \mathrm{G} 2468 \mathrm{a}, 4 \mathrm{G} 2468 \mathrm{~b}$ \\
6 & $6 \mathrm{G} 6$ & & $6 \mathrm{G} 468$ & \\
8 & $8 \mathrm{G} 8$ & & & $8 \mathrm{G} 2468 \mathrm{a}, 8 \mathrm{G} 2468 \mathrm{~b}$ \\
\hline
\end{tabular}

Table 19: Interpretations regarding some aspects of G-family solutions

\begin{tabular}{cl}
\hline Aspect & Interpretation \\
\hline$\Gamma=2$ & Charge \\
$\Gamma=24$ & Magnetic dipole moment \\
$\Gamma=246$ & Quadrupole distribution with respect to $4 \in \Gamma$ \\
$8 \in \Gamma$ & Rotation \\
\hline
\end{tabular}

We do not know of a reason for proposing that the series $2 \mathrm{G} \Gamma, 4 \mathrm{G} \Gamma, \ldots$ ends with $8 \mathrm{G} \Gamma$. We do not de-emphasize solutions $\Sigma$ G $\Gamma$ for which $10 \leq \Sigma \leq 20$.

Table 18 lists G-family solutions $\Sigma$ G $\Gamma$ for which $\Sigma$ appears in the list $\Gamma$. The expressions $|-2+4-6+8|$ and $|-2-4-6+8|$ show that two solutions comport with the notion of $4 \mathrm{G} 2468$. The expressions $|+2+4-6+8|$ and $|-2-4+6+8|$ show that two solutions comport with the notion of $8 \mathrm{G} 2468$. We use the symbol $\Sigma \gamma$ to refer to the set of G-family solutions $\Sigma G \Gamma$ for which $\Sigma$ appears in the list $\Gamma$. (See equation (15).) We use the symbol $\gamma \lambda$ to refer to the set of G-family solutions $\Sigma$ G $\Gamma$ for which $\lambda \neq \Sigma$ and $\lambda$ appears in the list $\Gamma$. (See equation (16).)

$$
\begin{gathered}
\Sigma \gamma=\{\Sigma \mathrm{G} \Gamma \mid \Sigma \in \Gamma\} \\
\gamma \lambda=\{\Sigma \mathrm{G} \Gamma \mid \lambda \neq \Sigma, \lambda \in \Gamma\}
\end{gathered}
$$

Proposed theory correlates the two-word term basic gravity with the $4 \mathrm{G} 4$ solution. Proposed theory correlates the three-word term dark energy forces with the 4G48, 4G246, 4G2468a, and 4G2468b solutions.

Table 19 discusses aspects of table 18. Here, we use wording that dovetails with classical physics (and not necessarily with quantum physics). Here, we use wording that dovetails with Newtonian modeling.

The following statements pertain regarding $2 \mathrm{G}$ and $4 \mathrm{G}$. Solution $2 \mathrm{G} 2$ correlates with a monopole interaction with charge. Solution 2G24 correlates with a dipole interaction with magnetic dipole moment. Solution 2G248 correlates with a quadrupole interaction with an object for which a non-zero magnetic dipole moment pertains, an axis of rotation pertains, and the axis of rotation does not match the axis correlating with the magnetic dipole moment. For the earth, the $2 \mathrm{G} 248$ interaction correlates with the non-alignment of the axis of rotation and the axis of the magnetic field. We posit that $8 \in \Gamma-$ or the number eight appearing in the list $\Gamma$ - correlates with non-zero rotation. Solution $4 \mathrm{G} 4$ correlates with a monopole interaction with non-rotational aspects of spherically symmetric mass (or of spherically symmetric rest energy). Solution $4 \mathrm{G} 48$ correlates with a dipole interaction with rotating spherically symmetric mass. Solution 4G246 correlates with a quadrupole interaction with non-rotational aspects of a distribution of mass for which the maximum moment of inertia does not equal the minimum moment of inertia. One of $4 \mathrm{G} 2468 \mathrm{a}$ and $4 \mathrm{G} 2468 \mathrm{~b}$ interacts with rotational aspects of quadrupole distributions of mass based on an axis of maximal moment of inertia. The other of 4G2468a and 4G2468b interacts with rotational aspects of quadrupole distributions of mass based on an axis of minimal moment of inertia.

The above statements regarding $2 \mathrm{G}$ and $4 \mathrm{G}$ dovetail with concepts that equations (17) and (18) symbolize. In proposed theory, modeling regarding quantum states and excitations does not necessarily involve modeling pertaining to translational motion. Equation (17) pertains. (See table 11.) Equation (18) correlates with linking G-family physics to models for forces and translational motion. (See the above discussion regarding $2 \mathrm{G}$ and $4 \mathrm{G}$ and see, for example, table 17.) Another aspect of such linking correlates with kinematics conservation laws. (See discussion related to table 14.)

$$
\Sigma \mathrm{G} \leftrightarrow \text { quantum excitations }
$$

$\Sigma \mathrm{G} \Gamma \leftrightarrow$ a bridge between quantum excitations and kinematics forces 
Table 20: Possible correlations between 0G solutions and simple bosons not belonging to the G-family of long-range forces

\begin{tabular}{cccc}
\hline Solution & Boson & Subfamily & $j_{\lambda \in \Gamma}$ \\
\hline $0 \mathrm{G} \emptyset$ & 0I (or aye) & OI & 0 \\
$0 \mathrm{G} 246$ & $\mathrm{~W}$ & $2 \mathrm{~W}$ & 3 \\
$0 \mathrm{G} 268$ & $\mathrm{Z}$ & $2 \mathrm{~W}$ & 3 \\
$0 \mathrm{G} 2468$ & $\mathrm{H}^{0}$ (or, Higgs) & $0 \mathrm{H}$ & 4 \\
\hline
\end{tabular}

Regarding possible G-family interactions with simple particles, we combine aspects of equation (11), table 14, and table 17. We posit that TA-side aspects of table 14 and table 17 combine. For example, for 8G8, a TA-side $S U(11)$ symmetry would pertain. (In table 14, seven TA-side oscillators pertain. In table 17, five TA-side oscillators pertain. The tables share their respective $n_{T A 0}=0$ value. Seven plus five minus one is 11.) We posit that a combined symmetry of either $S U(9)$ or $S U(11)$ correlates with possible interactions with multicomponent objects and with no interactions with simple particles. For example, $2 \mathrm{G} 68$ can interact with an atom but not with an isolated electron. (We correlate $2 \mathrm{G} 68$ with at least the 21-centimeter hyperfine interaction with hydrogen atoms. See discussion related to equation (94).) Each item that appears in table 17 and has a TA-side symmetry of $S U(5)$ or $S U(7)$ does not interact with simple particles. Each item that appears in table 17 and has a TA-side symmetry of None or $S U(3)$ can interact with simple particles.

Table 20 posits correlations between simple bosons and the $\Sigma=0$ solutions that correlate with table 17. We posit that the $\mathrm{W}$ boson correlates with 0G246 and not with 0G268. This assumption correlates with the notion of isomers of charged simple particles. (See discussion related to equation (83).) To the extent that table 20 pertains, G-family solutions point to all $\sigma=+1$ simple bosons and long-range forces. Here, $j_{\lambda \in \Gamma}$ denotes the number of elements in $\Gamma$ (as in $\Sigma G \Gamma$ ).

Each of the $\Sigma=0$ items that table 17 lists has a TA-side symmetry of none or $S U(3)$. Each one of the aye, W, Z, and Higgs bosons can interact with simple particles.

We posit that $\gamma \lambda$ items that appear in table 17 and have a TA-side symmetry listing (in table 17) that is not $S U(5)$ and is not $S U(7)$ correlate with the concept of anomalous moments for simple particles. (Regarding the notation $\gamma \lambda$, see equation (16).) For example, each one of solutions 4G26, 6G24, and 8G26 belongs to $\gamma 2$ and correlates with anomalous magnetic dipole moment. The ongoing theory Standard Model suggests computations whereby the anomalous magnetic dipole moment for a charged lepton is a sum of terms. The first term is $\alpha /(2 \pi)$. The symbol $\alpha$ denotes the fine-structure constant. (See equation (56).) The second term is proportional to $\alpha^{2}$. The third term is proportional to $\alpha^{3}$. Solution $4 \mathrm{G} 26$ might correlate with the $\alpha /(2 \pi)$ term that ongoing theory provides for charged leptons. Solution $6 \mathrm{G} 24$ features $4 \in \Gamma$ and varies with charged lepton generation. Solution 6G24 might correlate with a term that is proportional to $\alpha^{2} /(2 \pi)$. (See equation (66), equation (67), and discussion regarding table 36 . Note the result $\alpha^{2} /(2 \pi) \approx 8.48 \times 10^{-6}$.) Solution $8 \mathrm{G} 26$ might correlate with a term proportional to $\alpha^{3} /(2 \pi)$. Generally, $\Sigma \mathrm{G} 2$ might contribute a term proportional to $\alpha^{(\Sigma-2) / 2}$. Each anomalous dipole moment term correlates with the two values of $\lambda$ that $\Gamma$ includes. Each anomalous moment term correlates with an anomalous moment with respect to each of the two aspects that correlates with a $\Sigma$ G for which $\Sigma \in \Gamma$.

We explore the topic of object properties that correlate with $6 \mathrm{G}$ and $8 \mathrm{G}$.

Equation (19) provides ongoing theory limits for the sum, across three generations, of neutrino masses. (See reference [17]. Reference [29] provides the lowest of the upper limits that reference [17] lists.) Equation (19) comes from interpretations of astrophysics data. Equation (19) contrasts with some aspects of the ongoing theory elementary particle Standard Model that suggest that each one of the three neutrinos has zero mass.

$$
0.06 \mathrm{eV} / c^{2} \lesssim \sum_{j=1}^{3} m_{j} \lesssim 0.12 \mathrm{eV} / c^{2}
$$

We explore two sets of assumptions regarding choices of modeling.

First, we assume the ongoing theory notion that neutrino oscillations correlate with interactions that we correlate with the $4 \mathrm{G}$ subfamily. We assume that modeling correlating with table 11 pertains. Aspects of table 17 suggest the following. Each one of 4G4, 4G26, and 4G268 correlates with TA-side $S U(3)$ symmetry. The $S U(3)$ symmetry correlates with approximate conservation of fermion generation. Interactions correlating with $4 \mathrm{G} 4,4 \mathrm{G} 26$, or $4 \mathrm{G} 268$ do not catalyze neutrino oscillations. The solution 4 G48 correlates with TA-side $S U(5)$ symmetry. Simple particles do not interact directly with $4 \mathrm{G} 48$ long- 
Table 21: Interpretations regarding some aspects of G-family solutions

\begin{tabular}{cl}
\hline Aspect & Interpretation \\
\hline 8G & Interacts with lepton number minus baryon number \\
8G2468a and 8G2468b & Interact with individual neutrinos \\
8G2468a and 8G2468b & Catalyze neutrino oscillations \\
8G2468a and 8G2468b & $\begin{array}{l}\text { Catalyze effects that people interpret as implying at least one } \\
\text { non-zero neutrino mass }\end{array}$ \\
\hline
\end{tabular}

range forces. Interactions correlating with $4 \mathrm{G} 48$ do not catalyze neutrino oscillations. Solution 4G2468a and 4G2468b correlate with no TA-side symmetry. Interactions correlating with 4G2468a and 4G2468b might catalyze neutrino oscillations.

Second, we assume a proposed theory notion that $n_{S A 0}=-1$ correlates with zero rest energy. Modeling that does not include kinematics suggests that neutrinos do not interact with $4 \gamma$. Modeling suggests that interactions between neutrinos and $4 \gamma$ do not produce neutrino oscillations. Modeling suggests that interactions between neutrinos and $4 \mathrm{G}$ do not produce astrophysical effects that ongoing theory correlates with a non-zero sum of neutrino masses.

Independent of assumptions about modeling, equation (20) pertains. Here, the factor of three might correlate with the range $1 \leq j \leq 3$ in equation (19). Equation (20) may reflect equation (19) and a notion that some interaction strengths scale in proportion to $\alpha^{\Sigma / 2}$. The factor $\alpha^{2}$ in equation (20) correlates with $\alpha^{4} / \alpha^{2}$.

$$
3 \alpha^{2} m_{e} \approx 0.0816 e V / c^{2}
$$

Table 21 posits modeling that reconciles discussion above, equation (19), and equation (20). Table 21 extends table 19. The notion that positive numbers appear in equation (19) might suggest that, for interactions between two left-handed simple bosons, the combination of 8G2468a and 8G2468b is attractive.

We discuss possible implications regarding ongoing theory modeling. Ongoing theory astrophysics modeling does not include modeling that proposed theory correlates with $6 \mathrm{G}$ and $8 \mathrm{G}$. We posit one or two conceptual mapping steps. First, in the context of proposed theory, modeling for 8G octupole forces maps to modeling for $4 \mathrm{G}$ octupole dark energy forces. Perhaps that step suffices. In this context, ongoing theory modeling paralleling aspects of proposed theory 4G2468a and 4G2468b interprets 8G effects on neutrinos as correlating with mass. The following (or, second) step might pertain to the extent that relevant ongoing theory modeling does not correlate with proposed theory $4 \mathrm{G}$ dark energy forces. Second, in the context of proposed theory, modeling for $4 \mathrm{G}$ octupole forces maps to modeling involving $4 \mathrm{G} 4$. In this context, ongoing theory modeling based on only proposed theory 4G4 interprets $8 \mathrm{G}$ effects on neutrinos as correlating with mass.

We perform a check regarding reasonableness of proposed theory regarding interactions that couple to lepton number. We consider our interpretation of aspects of ongoing theory. We consider gravitational interactions between two electrons. Equation (21) describes results based just on the component that correlates with proposed theory 4G4 effects. Equation (22) assumes that $\epsilon$ correlates with one standard deviation regarding the mass of an electron. (Reference [17] provides the data that we use for these calculations.) The lepton number for an electron equals the lepton number for a matter neutrino. Equation (23) correlates with results based just on the component that correlates with proposed theory $8 \mathrm{G}$ effects. The result that equation (23) shows is less than the result that equation (22) shows. In this context of ongoing theory, the interaction, between two electrons, based on lepton number is not incompatible with measurements of electron masses.

$$
\begin{gathered}
G_{N}\left(m_{e}(1+\epsilon)\right)^{2} / r^{2} \approx G_{N}\left(m_{e}\right)^{2}(1+2 \epsilon) / r^{2} \\
|\epsilon| \approx 1.2 \times 10^{-8} \\
\left(\alpha^{2}\right)^{2} \approx 2.8 \times 10^{-9}
\end{gathered}
$$

Proposed theory suggests that, for Newtonian modeling, the strength of interactions with lepton number scales as $r^{-5}$. The strength of interactions with charge scales as $r^{-2}$. People might want to estimate a minimum energy for which the interaction between two charged leptons exhibits measurable effects of $8 \mathrm{G}$ octupole forces. 
Table 22: Changes, to representations, to reflect approximate conservation laws regarding baryon number and lepton number

(a) Changes regarding simple bosons having non-zero charge and regarding simple fermions

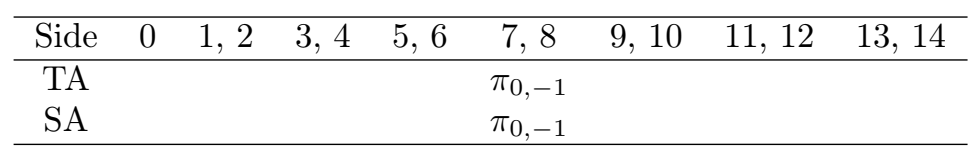

(b) Changes regarding other simple particles

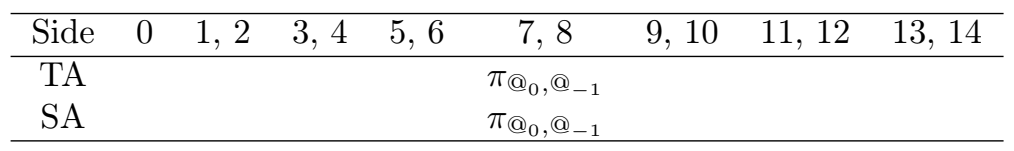

Table 23: Ground state for T-family bosons

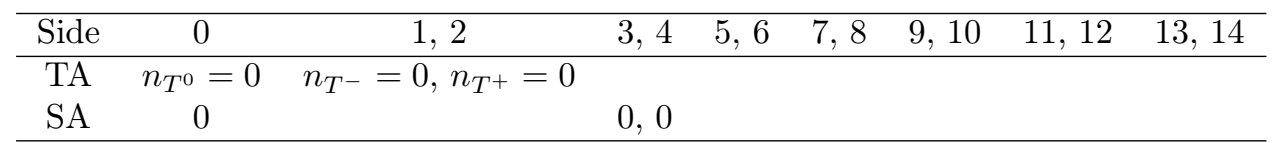

\subsection{Conservation of lepton number minus baryon number}

This unit shows modeling that correlates with conservation of lepton number minus baryon number.

Equation (24) shows a conserved quantity, $N_{L-B}$ (or, lepton number minus baryon number). Here, $L$ denotes lepton number and $B$ denotes baryon number. For a matter lepton, $L=+1$ and $B=0$. For an antimatter lepton, $L=-1$ and $B=0$. For a matter quark, $L=0$ and $B=1 / 3$. For an antimatter quark, $L=0$ and $B=-1 / 3$. For simple bosons and long-range forces, $0=L=B=N_{L-B}$.

$$
N_{L-B}=L-B
$$

Each of equation (98) and equation (100) shows an interaction that does not conserve $L$ and does not conserve $B$.

Table 22 shows changes, to representations, to reflect conservation of lepton number minus baryon number. Each change that table 22 shows satisfies $\widehat{A}_{X A}^{A L G}=0$. (Compare with table 4.) Regarding gluons (or, $2 \mathrm{U}$ simple bosons), information in table $22 \mathrm{~b}$ pertains independently from information that table $13 \mathrm{~b}$ shows.

We discuss T-family excitations.

Table 23 posits a ground state for T-family bosons. (Compare with table 6.) The magnitude of the charges of the $\mathrm{T}^{ \pm}$boson is one-third the magnitude of the charge of an electron. (See remarks regarding table 12.)

To describe $n$ excitations of the same state of one of the bosons, we use $n_{S A 0}=n=n_{T A}$, with TA _ correlating with the one boson. Other types of multiple excitations can pertain. We consider three excitations, each to a different state, of one of $\mathrm{T}^{-}$and $\mathrm{T}^{+}$. The states might differ by spin-orientation. (The states might differ by momentum. However, so far, we have de-emphasized discussing translational motion.) Modeling for the interaction might correlate with exciting, by one unit, each one of three SAside oscillators. The three oscillators are the SA0 oscillator, the SA3 oscillator, and the SA4 oscillator. Baryon number increases or decreases by one. Lepton number increases or decreases respectively by one. Conservation of lepton number minus baryon number pertains.

This work might extend to the following concepts. For objects for which $\sigma=+1$, the minimum magnitudes of some non-zero quantities are $\left|q_{e}\right|$ for charge, one for lepton number, and one for baryon number. (Here, we consider that a proton or other hadron with no more than three quarks correlates with $\sigma=+1$.) For objects for which $\sigma=-1$, the minimum magnitudes of some non-zero quantities are $\left|q_{e}\right| / 3$ for charge and one-third for baryon number. (Non-zero lepton number pertains only to objects for which $\sigma=+1$.) Each of the quantities charge, lepton number, and baryon number is additive with respect to components of a multicomponent object. 
Table 24: Subfamilies

(a) Spin-zero simple bosons

\begin{tabular}{cccccccccc}
\hline$\Sigma \Phi$ & $\sigma$ & TA & TA & TA & TA & SA & SA & SA & SA \\
& & 5,6 & 3,4 & 1,2 & 0 & 0 & 1,2 & 3,4 & 5,6 \\
\hline $0 \mathrm{H}$ & +1 & & & $\pi_{@_{0}, @_{-1}}$ & 0 & 0 & $\pi_{@_{0}, @_{-1}}$ & & \\
$(0 \mathrm{P})$ & -1 & & & $\pi_{@_{0}, @_{-1}}$ & 0 & 0 & $\pi_{@_{0}, @_{-1}}$ & & \\
$0 \mathrm{I}$ & +1 & & & $\pi_{@_{0}, @_{-1}}$ & -1 & -1 & $\pi_{@_{0}, @_{-1}}$ & & \\
$(0 \mathrm{~K})$ & -1 & & & $\pi_{@_{0}, @_{-1}}$ & -1 & -1 & $\pi_{@_{0}, @_{-1}}$ & & \\
\hline
\end{tabular}

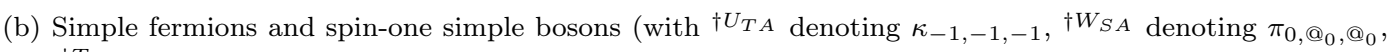
and $† T_{T A}$ denoting $\pi_{0, @_{0}, @_{0}}$ )

\begin{tabular}{cccccccccc}
\hline$\Sigma \Phi$ & $\sigma$ & $\mathrm{TA}$ & $\mathrm{TA}$ & $\mathrm{TA}$ & $\mathrm{TA}$ & $\mathrm{SA}$ & $\mathrm{SA}$ & $\mathrm{SA}$ & $\mathrm{SA}$ \\
& & 5,6 & 3,4 & 1,2 & 0 & 0 & 1,2 & 3,4 & 5,6 \\
\hline $1 \mathrm{~N}$ & +1 & & $\kappa_{@_{-1}, @_{-1}}$ & $\pi_{@_{0}, @_{-1}}$ & -1 & -1 & $\pi_{0,-1}$ & $\kappa_{-1,-1}$ & \\
$1 \mathrm{C}$ & +1 & & $\kappa_{@_{0}, @_{0}}$ & $\pi_{@_{0}, @_{-1}}$ & 0 & 0 & $\pi_{0,-1}$ & $\kappa_{0,0}$ & \\
$1 \mathrm{R}$ & -1 & & $\kappa_{-1,-1}$ & $\pi_{0,-1}$ & -1 & -1 & $\pi_{0,-1}$ & $\kappa_{@_{-1}, @_{-1}}$ & \\
$1 \mathrm{Q}$ & -1 & & $\kappa_{@_{0}, @_{0}}$ & $\pi_{0,-1}$ & 0 & 0 & $\pi_{0,-1}$ & $\kappa_{0,0}$ & \\
$2 \mathrm{U}$ & -1 & $\dagger U_{T A}$ & $\kappa_{@_{-1}, @_{-1}}$ & $\pi_{@_{0}, @_{-1}} \dagger U_{T A}$ & -1 & $\pi_{@_{0}, @_{-1}}$ & $\kappa_{@_{-1}, @_{-1}}$ & $\kappa_{@_{-1}, @_{-1}}$ \\
$2 \mathrm{~W}$ & +1 & & $\kappa_{@_{0}, @_{0}}$ & $\pi_{@_{0}, @_{-1}}$ & 0 & $\dagger W_{S A}$ & $\dagger W_{S A}$ & & \\
$2 \mathrm{~T}$ & -1 & & & $\tau_{T A}$ & $\dagger T_{T A}$ & 0 & $\pi_{@_{0}, @_{-1}}$ & $\kappa_{@_{0}, @_{0}}$ & \\
\hline
\end{tabular}

(c) Long-range forces

\begin{tabular}{cccccccccc}
\hline$\Sigma \Phi$ & $\sigma$ & TA & TA & TA & TA & SA & SA & SA & SA \\
& & 5,6 & 3,4 & 1,2 & 0 & 0 & 1,2 & 3,4 & $\cdots$ \\
\hline $2 \mathrm{G}$ & +1 & & & $\pi_{@_{0}, @_{-1}}$ & 0 & -1 & $\pi_{0, @_{0}}$ & & \\
$4 \mathrm{G}$ & +1 & & & $\pi_{@_{0}, @_{-1}}$ & 0 & -1 & & $\pi_{0, @_{0}}$ & \\
$\cdots \mathrm{G}$ & +1 & & & $\pi_{@_{0}, @_{-1}}$ & 0 & -1 & & & $\cdots$ \\
\hline
\end{tabular}

\subsection{A table of subfamilies of simple particles and long-range forces}

This unit recaps subfamilies of simple particles and long-range forces.

Table 24 alludes to all, but does not directly show some, ALG solutions that proposed theory suggests have physics-relevance regarding simple particles and long-range forces. (See table 1 and discussion that follows table 1.) Table 24 shows ground states. The table does not show information pertaining to conservation of lepton number minus baryon number. (See table 22.) Table 24a shows spin-zero simple bosons. Conservation of charge pertains for each spin-zero simple boson. Each one of the 0H and 0I bosons does not interact with multicomponent objects. Each one of the $0 \mathrm{P}$ and $0 \mathrm{~K}$ bosons does not necessarily interact with individual simple particles. Table $24 \mathrm{~b}$ shows simple fermions and spin-one simple bosons. The table shows information regarding charge, generation, and color charge. Conservation of charge pertains throughout the table. Table 24c shows information about long-range forces. Conservation of charge pertains throughout the table. Some components of some long-range forces do not interact with individual simple particles. Approximate conservation of generation pertains for some components of some long-range forces.

\subsection{Bases for proposed field theories}

This unit discusses harmonic oscillator mathematics that can underlie proposed field theories. This unit previews the notion that the mathematics underlies modeling that correlates with known and suggested simple particles and with known and suggested long-range forces.

Mathematics and ongoing theory include partial differential equations pertaining to isotropic harmonic oscillators. A partial differential equation correlating with an isotropic multidimensional quantum harmonic oscillator includes an operator that correlates with $r^{-2}$ and an operator that correlates with $r^{2}$. Here, $r$ denotes a radial spatial coordinate. (See equations (26) and (27).) The $r^{-2}$ operator might model aspects correlating with the square of an electrostatic potential. The potential correlates with $r^{-1}$. The force correlates with $r^{-2}$. The $r^{-2}$ operator might model aspects correlating with the square of a gravitational potential. The $r^{-2}$ operator might model aspects correlating with each long-range force 
$\Sigma$ G. (See table 11.) The $r^{-2}$ operator might model aspects correlating with excitations that pertain for each long-range force $\Sigma \mathrm{G}$ and that, thereby, have relevance for each long-range force component $\Sigma$ G $\Gamma$. The $r^{2}$ operator in equation (26) might model aspects correlating with the square of a strong interaction potential. This strong interaction potential would correlate with excitations related to the $2 \mathrm{U}$ subfamily (or, gluons) and with interactions within hadron-like particles. (Ongoing theory includes within the twoword term strong force the notion of a residual strong force. The three-word term residual strong force pertains to interactions between hadron-like particles. Proposed theory suggests correlating the residual strong force with the $0 \mathrm{P}$ - or, pie - simple boson and the $0 \mathrm{~K}$ - or, cake - simple boson.) Ongoing theory includes the concept of asymptotic freedom. The potential correlates with $r^{1}$. The force correlates with $r^{0}$.

Above, we find that ALG modeling related to the G-family might point to results about $\sigma=+1$ simple bosons that are not G-family bosons. Here, we explore the notion that PDE modeling might point to results pertaining to other than the G-family and the $2 \mathrm{U}$ subfamily. For example, operator aspects that correlate with $r^{0}$ might correlate with aspects of the weak interaction. Operator aspects that correlate with $r^{0}$ might correlate with aspects of the residual strong interaction (or, residual strong force).

Proposed theory includes modeling based on an analog, equation (25), to equation (1). Each of $A_{T A}^{P D E}$ and $A_{S A}^{P D E}$ is a quantum operator.

$$
0=A^{P D E}=A_{T A}^{P D E}-A_{S A}^{P D E}
$$

The following perspective pertains.

Equations (26) and (27) correlate with an isotropic quantum harmonic oscillator. Here, $r$ denotes the radial coordinate and has dimensions of length. The parameter $\eta_{S A}$ has dimensions of length. The parameter $\eta_{S A}$ is a non-zero real number. The magnitude $\left|\eta_{S A}\right|$ correlates with a scale length. The positive integer $D$ correlates with a number of dimensions. Each of $\xi_{S A}$ and $\xi_{S A}^{\prime}$ is a constant. (For an example of a physics centric use of the symbol $\xi_{S A}^{\prime}$, see discussion related to equation (107).) The symbol $\Psi(r)$ denotes a function of $r$ and, possibly, of angular coordinates. The symbol $\nabla_{r}^{2}$ denotes a Laplacian operator. In some ongoing theory applications, $\Omega_{S A}$ is a constant that correlates with aspects correlating with angular coordinates. Our discussion includes the term $\Omega_{S A}$ and, otherwise, tends to de-emphasize some angular aspects. We associate the term SA-side with this use of symbols and mathematics, in anticipation that the symbols used correlate with spatial aspects of physics modeling and in anticipation that TA-side symbols and mathematics pertain for some modeling.

$$
\begin{gathered}
\xi_{S A} \Psi(r)=\left(\xi_{S A}^{\prime} / 2\right)\left(-\left(\eta_{S A}\right)^{2} \nabla_{r}^{2}+\left(\eta_{S A}\right)^{-2} r^{2}\right) \Psi(r) \\
\nabla_{r}^{2}=r^{-(D-1)}(\partial / \partial r)\left(r^{D-1}\right)(\partial / \partial r)-\Omega_{S A} r^{-2}
\end{gathered}
$$

Including for $D=1$, each of equation (26), equation (27), and the function $\Psi$ pertains for the domain that equation (28) shows. (We de-emphasize exploration of possible solutions for $D \leq 0$.)

$$
0<r<\infty
$$

We consider solutions of the form that equation (29) shows. (For $\nu_{S A}<0$, this work pertains for the domain that equation (28) defines. For $\nu_{S A} \geq 0$, this work might pertain for the domain $0 \leq r<\infty$. For $\nu_{S A} \geq 0$ and $r=0$, angular aspects, $Y$, of $\Psi \propto \phi(r) Y$ (angular coordinates) might be undefined. People might ignore that lack of definition, based on the notion that, for cases in which $Y$ is undefined, $\phi(0)=0$.

$$
\Psi(r) \propto\left(r / \eta_{S A}\right)^{\nu_{S A}} \exp \left(-r^{2} /\left(2\left(\eta_{S A}\right)^{2}\right)\right), \text { with }\left(\eta_{S A}\right)^{2}>0
$$

Equations (30) and (31) characterize solutions. The parameter $\eta_{S A}$ does not appear in these equations. Equation (32) correlates with the domains of $D$ and $\nu_{S A}$ for which normalization pertains for $\Psi(r)$. For $D+2 \nu_{S A}=0$, normalization pertains in the limit $\left(\eta_{S A}\right)^{2} \rightarrow 0^{+}$. Regarding mathematics relevant to normalization for $D+2 \nu_{S A}=0$, the delta function that equation (33) shows pertains. Here, $x^{2}$ correlates with $r^{2}$ and $4 \epsilon$ correlates with $\left(\eta_{S A}\right)^{2}$. Reference [30] provides equation (33). The difference in domains, between $-\infty<x<\infty$ and equation (28), is not material here. (Our use of this type of modeling features normalization. Considering normalization leads to de-emphasizing possible concerns, about variations - as a function of angular coordinates - as $r$ approaches zero, regarding $Y$ (angular coordinates). Considering normalization leads to de-emphasizing possible concerns, regarding singularities as $r$ approaches zero, regarding some $\Psi(r)$.) 


$$
\begin{gathered}
\xi_{S A}=\left(D+2 \nu_{S A}\right)\left(\xi_{S A}^{\prime} / 2\right) \\
\Omega_{S A}=\nu_{S A}\left(\nu_{S A}+D-2\right) \\
D+2 \nu_{S A} \geq 0 \\
\delta(x)=\lim _{\epsilon \rightarrow 0^{+}}(1 /(2 \sqrt{\pi \epsilon})) e^{-x^{2} /(4 \epsilon)}
\end{gathered}
$$

Some applications feature the numbers of dimensions that equations (34) and (35) show. Equation (34) correlates with a notion of three spatial dimensions. Equation (35) correlates with a notion of one temporal dimension.

$$
\begin{aligned}
& D_{S A}^{*}=3 \\
& D_{T A}^{*}=1
\end{aligned}
$$

We anticipate using equations (36) and (37). Here, each of $2 S$ and $2 S^{\prime}$ is a nonnegative integer. The case that features equation (36), $\sigma=+1$, and $S=\nu_{S A}$ is a restating of equation (31). The case that features equation (36) and $\sigma=-1$ correlates with some aspects of proposed theory modeling. (See discussion related to equation (38).) Similar concepts pertain regarding equation (37) and $\sigma^{\prime}$.

$$
\begin{gathered}
\Omega_{S A}=\sigma S\left(S+D_{S A}^{*}-2\right)=\sigma S(S+1), \text { for } \sigma= \pm 1 \\
\Omega_{T A}=\sigma^{\prime} S^{\prime}\left(S^{\prime}+D_{T A}^{*}-2\right)=\sigma^{\prime} S^{\prime}\left(S^{\prime}-1\right), \text { for } \sigma^{\prime}= \pm 1
\end{gathered}
$$

The following notions pertain.

- The symbol $S$ can correlate with ongoing theory notions of spin divided by $\hbar$. The symbol $\hbar$ denotes the reduced Planck's constant.

- For some solutions - which comport with equation (36) - to equation $(31), D \neq D_{S A}^{*}$.

- Solutions for which $\nu_{S A}=-1 / 2$ can correlate with notions of fields for simple fermions.

- Solutions for which $\nu_{S A}=-1$ can correlate with notions of fields for simple bosons.

- Solutions for which $\nu_{S A}=-3 / 2$ can correlate with notions of particles for simple fermions.

- TA-side PDE solutions are radial with respect to $t$, the TA-side analog to the SA-side radial coordinate $r$.

- For some solutions, $D \neq D_{T A}^{*}$.

Some applications feature a notion of $D^{\prime \prime}=2$. For these cases, we, in effect, separate some PDE aspects into PDE aspects correlating with oscillator pairs. Examples of such oscillator pairs include the TA0and-SA0 oscillator pair and the SA1-and-SA2 oscillator pair.

- For some cases correlating with $D_{T A}^{*}=1$ and $D_{S A}^{*}=3, D^{\prime \prime}=2$ pertains for each of the TA0-andSA0 oscillator pair and the SA1-and-SA2 oscillator pair.

- Solutions for which $\nu_{T A 0, S A 0}=\nu_{S A 1, S A 2}=-1$ can correlate with notions of particles for simple bosons.

Table 25 provides details leading to equations (30) and (31). We consider equations (26), (27), and (29). The table assumes, without loss of generality, that $\left(\xi_{S A}^{\prime} / 2\right)=1$ and that $\eta_{S A}=1$. More generally, we assume that each of the four terms $K$ and each of the two terms $V$ includes appropriate appearances of $\left(\xi_{S A}^{\prime} / 2\right)$ and $\eta_{S A}$. The term $V_{+2}$ correlates with the right-most term in equation (26). The term $V_{-2}$ correlates with the right-most term in equation (27). The four $K$ terms correlate with the other term in equation (27). The sum of the two $K_{0}$ terms correlates with the factor $D+2 \nu_{S A}$ in equation (30).

We discuss PDE modeling that correlates with the notions that $\Omega_{T A}$ is nonpositive, $\sigma^{\prime}=-1, \Omega_{S A}$ is nonpositive, and $\sigma=-1$. 
Table 25: Terms correlating with an SA-side PDE equation (assuming that $\left(\xi_{S A}^{\prime} / 2\right)=1$ and $\eta_{S A}=1$ )

\begin{tabular}{ccccc}
\hline Term $/ \exp \left(-r^{2} / 2\right)$ & $\begin{array}{c}\text { Symbol } \\
\text { for term }\end{array}$ & $\begin{array}{c}\text { Change in } \\
\text { power of } r\end{array}$ & Non-zero unless ... & Notes \\
\hline$-r^{\nu_{S A}+2}$ & $K_{+2}$ & +2 & - & Cancels $V_{+2}$ \\
$\left(D+\nu_{S A}\right) r^{\nu_{S A}}$ & $K_{0 a}$ & 0 & $D+\nu_{S A}=0$ & - \\
$\nu_{S A} r^{\nu_{S A}}$ & $K_{0 b}$ & 0 & $\nu_{S A}=0$ & - \\
$-\nu_{S A}\left(\nu_{S A}+D-2\right) r^{\nu_{S A}-2}$ & $K_{-2}$ & -2 & $\nu_{S A}=0$ or & Cancels $V_{-2}$ \\
& & & $\left(\nu_{S A}+D-2\right)=0$ & \\
$\Omega_{S A} r^{\nu_{S A}-2}$ & $V_{-2}$ & -2 & $\Omega_{S A}=0$ & Cancels $K_{-2}$ \\
$r^{\nu_{S A}+2}$ & $V_{+2}$ & +2 & - & Cancels $K_{+2}$ \\
\hline
\end{tabular}

Proposed theory includes PDE modeling for which equation (38) pertains regarding the TA0-and-SA0 oscillator pair. (See, for example, equation (108).) These applications correlate with the notion that, for equation (25) and appropriate assumptions, one can move, in equation (25), the originally nonnegative $\Omega_{T A} / t^{2}$ term from correlating with $A_{T A}^{P D E}$ to become a nonpositive $\Omega_{S A} / r^{2}$ term correlating with $A_{S A}^{P D E}$ and one can move the originally nonnegative $\Omega_{S A} / r^{2}$ term from correlating with $A_{S A}^{P D E}$ to become a nonpositive $\Omega_{T A} / t^{2}$ term correlating with $A_{T A}^{P D E}$. After the moves, $\Omega_{T A}$ is nonpositive, $\sigma^{\prime}=-1, \Omega_{S A}$ is nonpositive, and $\sigma=-1$. The assumptions include equation (39). Equation (40) defines $v_{c}$.

$$
\begin{gathered}
t^{2} /\left(2\left(\eta_{T A}\right)^{2}\right)+r^{2} /\left(2\left(\eta_{S A}\right)^{2}\right)=\operatorname{tr} /\left(\left|\eta_{T A}\right| \cdot\left|\eta_{S A}\right|\right) \\
t /\left(\left|\eta_{T A}\right|\right)=r /\left(\left|\eta_{S A}\right|\right) \\
v_{c}=\left|\eta_{S A}\right| /\left|\eta_{T A}\right|
\end{gathered}
$$

PDE-based modeling might correlate with some aspects of unification of the strong, electromagnetic, and weak interactions. We consider modeling for which $2 \nu_{S A}$ is a non-negative integer. Based on the $r^{-2}$ spatial factor, the $V_{-2}$ term might correlate with the square of an electrostatic potential. Based on the $r^{2}$ spatial factor, the $V_{+2}$ term might correlate (at least, within hadrons) with the square of a potential correlating with the strong interaction. The sum $K_{0 a}+K_{0 b}$ might correlate with the strength of the weak interaction. (The effective range of the weak interaction is much smaller than the size of a hadron. Perhaps, the spatial characterization $r^{0}$ correlates with an approximately even distribution, throughout a hadron, for the possibility of a weak interaction occurring.) Based on the $V_{-2}$ term, we expect that $\xi_{S A}^{\prime}$ includes a factor $\hbar^{2}$. The choice between $\sigma=+1$ and $\sigma=-1$ allows for modeling each one of electrostatic repulsion and electrostatic attraction.

We contrast aspects and uses of QFTs (or, quantum field theories). Ongoing theory QFT features modeling that is linear in energy. Models explain or predict energies that systems exhibit. Proposed theory QFT features modeling that is quadratic in energy. Models explain or predict states or objects that nature embraces. Proposed theory QFT includes so-called branch points. Each branch point might point to aspects of ongoing QFT. Each branch point might point to a path from proposed QFT to details of ongoing QFT.

We note a possible aspect of synergy between ongoing QFT models that comport with special relativity and proposed QFT. For zero-mass entities, an excitation correlates with adding a unit of each of energy and momentum. For zero-mass entities, an excitation correlates with adding a unit of each of square of energy and square of momentum.

This essay de-emphasizes thoroughly discussing the extent to which each of $t$ and $r$, as used above, correlates with coordinates that people might use for, respectively, temporal and spatial aspects of ongoing theory dynamics modeling regarding motion within systems. (See, for example, equation (38).) This essay de-emphasizes discussing the extent to which each of $t$ and $r$, as used above, correlates with coordinates that people might use for, respectively, temporal and spatial aspects of ongoing theory kinematics modeling. This essay de-emphasizes exploring the extent to which allowing for variation, regarding $\eta_{S A}$ or $\eta_{T A}$, with respect to ongoing theory notions of time or proper time leads to useful models regarding diffusion. 
Table 26: Interaction vertices for interactions involving only simple particles and long-range forces

\begin{tabular}{lcl}
\hline Interaction & Effective $\nu$ & Example \\
\hline $0 \mathrm{f} 1 \mathrm{~b} \leftrightarrow 2 \mathrm{f} 0 \mathrm{~b}$ & -1 & $\mathrm{~A} \mathrm{Z}$ boson creates a matter-and-antimatter pair of fermions. \\
$1 \mathrm{f} 1 \mathrm{~b} \leftrightarrow 1 \mathrm{f} 1 \mathrm{~b}$ & $-3 / 2$ & An electron and a $\mathrm{W}^{+}$boson produce a neutrino. \\
$1 \mathrm{f} 1 \mathrm{~b} \leftrightarrow 3 \mathrm{f} 0 \mathrm{~b}$ & $-3 / 2$ & $1 \mathrm{C}^{+1}+1 \mathrm{R}^{0}+1 \mathrm{Q}^{-2 / 3} \rightarrow 1 \mathrm{Q}^{+2 / 3}+2 \mathrm{~T}^{-1 / 3}$. \\
$0 \mathrm{fnb} \leftrightarrow 0 \mathrm{fnb}$, for $\mathrm{n} \geq 2$ & $-n$ & A Higgs boson creates two photons. \\
\hline
\end{tabular}

\subsection{Interaction vertices}

This unit catalogs interaction vertices for interactions that involve only simple particles and long-range forces.

The following remarks pertain regarding interaction vertices that model interactions that involve simple particles and long-range forces. These remarks extend discussion related to equation (36).

Table 26 lists types of interaction vertices that proposed theory includes. Here, in the symbol nf, $\mathrm{n}$ denotes a number of simple fermions. In the symbol $\mathrm{nb}, \mathrm{n}$ denotes a number of simple bosons and long-range forces. A symbol of the form $a \leftrightarrow b$ denotes two cases, namely $a \rightarrow b$ and $b \rightarrow a$. A symbol of the form $\mathrm{a} \rightarrow \mathrm{b}$ denotes the notion that the interaction de-excites each component of a by one unit and excites each component of $\mathrm{b}$ by one unit. (Note, for example, that de-excitation of a photon mode does not necessarily produce a ground state.) For each type of interaction vertex, the effective $\nu$ is the sum, over incoming field solutions, of the relevant $\nu_{-}$and is also the sum, over outgoing field solutions, of the relevant $\nu_{-}$. (Technically, here we might need to assume that $\nu_{-}$for each of gluons and long-range forces is the same as $\nu_{-}$for weak interaction bosons.) We note that, in effect, the value of effective $\nu$ correlates with aspects of a product of solutions of the form that equation (29) shows. Ongoing theory includes (and table 26 mentions examples of) $1 \mathrm{f} 0 \mathrm{~b} \leftrightarrow 1 \mathrm{f} 1 \mathrm{~b}$ and $0 \mathrm{f} 1 \mathrm{~b} \leftrightarrow 0 \mathrm{f} 2 \mathrm{~b}$ interactions. Proposed theory can embrace ongoing theory $1 \mathrm{f} 0 \mathrm{~b} \leftrightarrow 1 \mathrm{f} 1 \mathrm{~b}$ interactions via the case of $1 \mathrm{f} 1 \mathrm{~b} \leftrightarrow 1 \mathrm{f} 1 \mathrm{~b}$ and the notion that the other boson correlates with 0I phenomena. (The symbol 0I denotes a zero-spin, zero-mass, zero-charge, $\sigma=+1$ simple boson that proposed theory suggests that nature might embrace. See table 1.) Proposed theory can embrace ongoing theory $0 \mathrm{f} 1 \mathrm{~b} \leftrightarrow 0 \mathrm{f} 2 \mathrm{~b}$ interactions via the case of $0 \mathrm{f} 2 \mathrm{~b} \leftrightarrow 0 \mathrm{f} 2 \mathrm{~b}$ and the notion that the other boson correlates with 0I phenomena. Proposed theory modeling can embrace, at least regarding $0 \mathrm{f} 1 \mathrm{~b} \leftrightarrow 0 \mathrm{fnb}$ cases in which the $1 \mathrm{~b}$ in $0 \mathrm{f} 1 \mathrm{~b}$ correlates with a non-zero-mass zero-charge simple boson, the notion of an effective $\nu$ of $-n$. Ongoing theory includes limits based on fermion statistics and does not necessarily include $1 \mathrm{f} 1 \mathrm{~b} \leftrightarrow 3 \mathrm{f} 0 \mathrm{~b}$ interactions. Table 26 shows an example of a $1 \mathrm{f} 1 \mathrm{~b} \leftrightarrow 3 \mathrm{f} 0 \mathrm{~b}$ interaction that might help catalyze baryon asymmetry. Here, the $1 \mathrm{C}$ particle can be a positron. The $1 \mathrm{Q}^{-2 / 3}$ particle can be an anti-up quark. The $1 \mathrm{Q}^{+2 / 3}$ particle can be an up quark. The $1 \mathrm{R}$ particle can be one of three similar simple fermions that proposed theory predicts. The $2 \mathrm{~T}$ particle is a simple boson that proposed theory predicts. (See discussion related to equation (98).) Here, the superscripts correlate with charge, in units of $\left|q_{e}\right|$ (or, in units of the magnitude of the charge of an electron). Here, each of the three $3 f$ fermions differs from the other two $3 \mathrm{f}$ fermions. Ongoing theory limitations based on fermion statistics do not necessarily pertain. (Also, ongoing theory might be able to model some proposed theory $1 \mathrm{f} 1 \mathrm{~b} \rightarrow 3 \mathrm{f} 0 \mathrm{~b}$ interactions via the sequence $1 \mathrm{f} 1 \mathrm{~b} \rightarrow 1 \mathrm{f} 1 \mathrm{~b}$ followed by $0 \mathrm{f} 1 \mathrm{~b} \rightarrow 2 \mathrm{f} 0 \mathrm{~b}$. Here, the outgoing $1 \mathrm{~b}$ in the first interaction becomes the incoming $1 \mathrm{~b}$ in the second interaction.)

Ongoing theory includes the following sequence of vertices. A fermion enters a $1 \mathrm{f} 0 \mathrm{~b} \rightarrow 1 \mathrm{f} 1 \mathrm{~b}$ vertex. The exiting fermion enters a $1 \mathrm{f} 0 \mathrm{~b} \rightarrow 1 \mathrm{f} 1 \mathrm{~b}$ vertex. The fermion exiting the second vertex enters a $1 \mathrm{f} 1 \mathrm{~b} \rightarrow 1 \mathrm{f} 0 \mathrm{~b}$ vertex that de-excites the boson that the first vertex excited. Modeling based on such a sequence can pertain for calculations of anomalous magnetic dipole moments. The boson is a photon. Some aspects of proposed theory do not necessarily include the notion of virtual particles and do not necessarily include such a sequence.

Proposed theory also points, for the weak interaction, to modeling that does not necessarily require the notion of virtual particles. Equation (41) shows an ongoing theory $1 \mathrm{f} 1 \mathrm{~b} \leftrightarrow 1 \mathrm{f} 1 \mathrm{~b}$ vertex. A muon transforms into a matter neutrino and a W boson. Equation (42) shows an ongoing theory $1 \mathrm{f} 1 \mathrm{~b} \leftrightarrow 1 \mathrm{f} 1 \mathrm{~b}$ vertex. The $\mathrm{W}$ boson transforms into an electron and an antimatter neutrino. Proposed theory can embrace the $1 \mathrm{f} 1 \mathrm{~b} \leftrightarrow 3 \mathrm{f} 0 \mathrm{~b}$ vertex that equation (43) shows. Here, the $0 \mathrm{~B}$ boson might be, for example, a OI boson. Equation (43) does not show a virtual particle such as a $\mathrm{W}$ boson.

$$
\begin{aligned}
& \mu^{-} \rightarrow \nu+W^{-} \\
& W^{-} \rightarrow e^{-}+\bar{\nu}
\end{aligned}
$$


Table 27: Relationships between some PDE parameters for $\Sigma \mathrm{W}, \Sigma \mathrm{H}, \Sigma \mathrm{I}, \Sigma \mathrm{P}, \Sigma \mathrm{K}$, and $\Sigma \mathrm{T}$ solutions

\begin{tabular}{ccccccccccc}
\hline$D_{S A}^{*}$ & $\nu_{S A}$ & $D_{S A}^{*}+2 \nu_{S A}$ & $D$ & $S$ & $\Omega$ & $\sigma$ & $D$ & $D+2 \nu_{S A}$ & $2 S+1$ & $\Sigma \Phi$ \\
\hline 3 & -1 & 1 & $3-\Omega$ & 1 & 2 & +1 & 1 & -1 & 3 & $2 \mathrm{~W}$ \\
3 & -1 & 1 & $3-\Omega$ & 0 & 0 & +1 & 3 & 1 & 1 & $0 \mathrm{H}, 0 \mathrm{I}$ \\
3 & -1 & 1 & $3-\Omega$ & 0 & 0 & -1 & 3 & 1 & 1 & $0 \mathrm{P}, 0 \mathrm{~K}$ \\
3 & -1 & 1 & $3-\Omega$ & 1 & -2 & -1 & 5 & 3 & 3 & $2 \mathrm{~T}$ \\
\hline
\end{tabular}

$$
\mu^{-}+0 \mathrm{~B} \rightarrow \nu+e^{-}+\bar{\nu}
$$

For proposed theory modeling of interactions that involve simple particles and long-range forces in free environments, the PDE notion of the mathematical limit expression $\left(\eta_{S A}\right)^{2} \rightarrow 0$ pertains. (See discussion related to equation (33).) Here, $\left(\eta_{T A}\right)^{2} \rightarrow 0$ pertains. We say that the vertex models as being point-like with respect to coordinates. Here, point-like refers to the temporal coordinate and refers to either a radial spatial coordinate or three spatial coordinates.

An example of modeling of interactions that involve simple particles in so-called confined environments might feature modeling regarding interactions with a quark that exists within a proton.

For proposed theory modeling of interactions that involve simple particles and long-range forces in confined environments, the PDE notion of $\left(\eta_{S A}\right)^{2}>0$ can pertain. (See discussion related to equation (38).) The expression that equation (44) shows might correlate with the size of the multicomponent object that correlates with the term confined environment. We say that the vertex models as being volumelike with respect to coordinates. Here, volume-like refers to, at least, either a radial spatial coordinate or three spatial coordinates. Volume-like correlates also with a non-point-like domain for the temporal coordinate.

$$
\left|\eta_{S A}\right|
$$

\subsection{Aspects regarding simple particles}

This unit discusses roles that PDE modeling plays regarding pointing to and describing simple particles other than gluons.

The following remarks illustrate roles, leading to table 1, for PDE modeling. The remarks support the notion that PDE modeling might output solutions that correlate with all simple particles other than U-family simple bosons. The remarks show aspects of using PDE notions regarding field solutions and particle solutions. (See remarks related to equation (29) and solutions for which $\nu_{S A}$ is one of $-1 / 2,-1$, and $-3 / 2$.)

Table 27 summarizes some simple-boson-centric PDE results for field centric solutions. Each solution correlates with $\nu_{S A}=-1$ and with a positive integer $D$. We feature solutions to equations (30) and (31). While $D$ need not equal three, each $\Omega_{S A}$ comports with $D_{S A}^{*}=3$ and with the requirement that $\Omega_{S A}=\sigma S(S+1)$. For each item that the table lists in the column labeled $\Sigma \Phi$, the number of possible particles, including antiparticles, equals $2 S+1$. For example, $2 \mathrm{~W}$ correlates, by this count, with three particles - the $\mathrm{Z}, \mathrm{W}^{+}$, and $\mathrm{W}^{-}$particles. We limit solutions for which $\sigma=+1$ to solutions for which $S \leq 1$. Any solutions for which $\sigma=+1$ and $S \geq 2$ would feature $D$ not being a positive integer. Each one of $2 \mathrm{U}$ solutions and $\Sigma \mathrm{G}$ solutions correlates with terms in the operators in equations (26) and (27) and does not appear in table 27.

Table 28 summarizes simple-fermion-centric PDE solutions. Per discussion related to equation (31), $\nu_{S A}=-1 / 2$ correlates with fields and $\nu_{S A}=-3 / 2$ correlates with particles. For each item that table 28 lists in the column labeled $\Sigma \Phi$, one of the following two sentences pertains. For $\sigma=+1,2 S+1$ equals the number of simple particles (including antiparticles) per generation. For $\sigma=-1,2 S+1$ equals half of the number of simple particles (including antiparticles) per generation.

We discuss the extent to which proposed theory should embrace $\sigma=-1$ solutions that tables 27 and 28 do not show.

PDE $\sigma=-1$ boson-centric solutions exist mathematically for each even integer $\Sigma$ that exceeds two. Such solutions might correlate with simple particles. PDE $\sigma=-1$ fermion-centric solutions exist mathematically for each odd integer $\Sigma$ that exceeds two. Pairs of such odd integer $\Sigma$ solutions might correlate with simple particles.

We note two cases related to data. For one case, models pertain to hadron-like particles or to atomic nuclei. For the other case, models pertain to other confined environments. One example of another 
Table 28: Fermion-centric PDE solutions

\begin{tabular}{ccccccccccc}
\hline$D_{S A}^{*}$ & $\nu_{S A}$ & $D_{S A}^{*}+2 \nu_{S A}$ & $D$ & $S$ & $\Omega$ & $\sigma$ & $D$ & $D+2 \nu_{S A}$ & $2 S+1$ & $\Sigma \Phi$ \\
\hline 3 & $-1 / 2$ & 2 & $(5-4 \Omega) / 2$ & $1 / 2$ & $3 / 4$ & +1 & 1 & 0 & 2 & $1 \mathrm{C}, 1 \mathrm{~N}$ \\
3 & $-3 / 2$ & 0 & $(21-4 \Omega) / 6$ & $1 / 2$ & $3 / 4$ & +1 & 3 & 0 & 2 & $1 \mathrm{C}, 1 \mathrm{~N}$ \\
3 & $-1 / 2$ & 2 & $(5-4 \Omega) / 2$ & $1 / 2$ & $-3 / 4$ & -1 & 4 & 3 & 2 & $1 \mathrm{Q}, 1 \mathrm{R}$ \\
3 & $-3 / 2$ & 0 & $(21-4 \Omega) / 6$ & $1 / 2$ & $-3 / 4$ & -1 & 4 & 1 & 2 & $1 \mathrm{Q}, 1 \mathrm{R}$ \\
\hline
\end{tabular}

Table 29: Rest energies for known non-zero-mass simple bosons

\begin{tabular}{|c|c|c|c|c|c|c|c|c|}
\hline \multirow{2}{*}{$\Phi$} & \multirow{2}{*}{$S$} & \multirow{2}{*}{$\sigma$} & \multirow{2}{*}{ Symbol } & \multirow{2}{*}{ Name } & \multirow{2}{*}{$\begin{array}{l}\text { Experimental } \\
m c^{2}(\mathrm{GeV})\end{array}$} & \multicolumn{2}{|c|}{ Calculated } & \multirow{2}{*}{$\begin{array}{c}\text { Difference } \\
\text { (standard deviations) }\end{array}$} \\
\hline & & & & & & $\mathrm{N}$ & $m c^{2}(\mathrm{GeV})$ & \\
\hline W & 1 & +1 & $2 \mathrm{~W} 1,2 \mathrm{~W} 2$ & $\mathrm{~W}$ & $80.379 \pm 0.012$ & 7 & 80.042 & $\approx 3.4$ \\
\hline $\mathrm{W}$ & 1 & +1 & 2W0 & $\mathrm{Z}$ & $91.1876 \pm 0.0021$ & 9 & 91.1876 & - \\
\hline $\mathrm{H}$ & 0 & +1 & $\mathrm{OHO}$ & $\mathrm{H}^{0}$ & $125.18 \pm 0.16$ & 17 & 125.33 & $\approx 1.0$ \\
\hline
\end{tabular}

confined environment might be a neutron star. We know of no phenomena that seem to require modeling that includes solutions for which $\sigma=-1$ and $\Sigma \geq 3$. We de-emphasize solutions for which $\sigma=-1$ and $\Sigma \geq 3$.

\subsection{Masses of the weak interaction bosons, the Higgs boson, and the 0I boson}

This unit shows modeling that links the masses of the weak interaction bosons, the Higgs boson, and the OI boson.

We explore relationships between masses of the $2 \mathrm{~W}$ (or, $\mathrm{W}$ and $\mathrm{Z}$ ), $0 \mathrm{H}$, and $0 \mathrm{I}$ bosons.

Table 29 shows rest energies for the known non-zero-mass simple bosons. (See reference [17].)

We discuss approximate ratios for the squares of masses of the Higgs, Z, and W bosons. The most accurately known of the three masses is the mass of the $\mathrm{Z}$ boson. Based on the ratios (of squares of masses) that equation (45) shows, the possibly least accurately suggested mass is that of the W boson. Equation (45) correlates with a number that is within four standard deviations of the nominal mass of the $\mathrm{W}$ boson. (See table 29.) Proposed theory correlates the numbers in equation (45) with, respectively, the expressions $17=17,9=10-1-0$, and $7=10-1-2$. Each of zero, one, two, 10, and 17 correlates with the value of $D+2 \nu$ for a PDE solution for which $D^{\prime \prime}=2$. (See table 30.) Equation (46) shows the size of one unit. The related mass is $30.396 \mathrm{GeV} / c^{2}$.

$$
\begin{gathered}
\left(m_{H^{0}}\right)^{2}:\left(m_{Z}\right)^{2}:\left(m_{W}\right)^{2}:: 17: 9: 7 \\
9.239 \times 10^{2}\left(\mathrm{GeV} / c^{2}\right)^{2}:: 1
\end{gathered}
$$

Table 30 summarizes mathematical results that correlate with $D^{\prime \prime}=2$. (Compare with tables 27 and 28.) Here, we correlate with $D^{\prime \prime}$ the symbols $S^{\prime \prime}, \Omega^{\prime \prime}$, and $\sigma^{\prime \prime}$. Each of $S^{\prime \prime}, \Omega^{\prime \prime}$, and $\sigma^{\prime \prime}$ does not necessarily correlate with uses of $S, S^{\prime}, \Omega, \sigma$, or $\sigma^{\prime}$ in models regarding simple particles. For $\Omega^{\prime \prime}=0$, the table uses the letters NR to denote that the sign of $\sigma^{\prime \prime}$ is not relevant.

The following correlations might pertain regarding relative squares of masses. (See table 20.) For each of the $\mathrm{W}, \mathrm{Z}$, and $\mathrm{OH}$ bosons, a positive term correlating with $\sigma^{\prime \prime}=-1$ and $S^{\prime \prime}=j_{\lambda \in \Gamma}$ pertains. For the $\mathrm{W}$ and $\mathrm{Z}$ bosons, a negative term correlates with spin-one and with a negative term correlating with $\sigma^{\prime \prime}=-1$ and $S^{\prime \prime}=0$. For the $\mathrm{W}$ boson, a negative term correlates with a charge of magnitude equal to the magnitude of the charge of the electron and with a negative term correlating with $\sigma^{\prime \prime}=-1$ and $S^{\prime \prime}=2$.

We explore concepts regarding $0 \mathrm{G} \emptyset$.

One might assume that the 0I solution correlates with $S^{\prime \prime}=j_{\lambda \in \Gamma}=0$. The result $S^{\prime \prime}=0$ correlates with a relative square of mass of one. The mass would approximately equal $30.4 \mathrm{GeV} / c^{2}$. We know of no observations that would support the existence of such a particle. We note that, for each of the W, Z, and Higgs bosons, the 0G $\Gamma$ solution has $n_{T A 0}=0$. (See table 17.) For the $0 \mathrm{G} \emptyset$ solution, $n_{T A 0}=-1$.

For each $\Sigma \geq 2 \Sigma G \Gamma$ solution that nature embraces, the mass is zero. We suggest that each solution correlates with $\sigma^{\prime \prime}=+1$ and $S^{\prime \prime}=1$. Per table 30, the relative mass correlates with $D+2 \nu=0$. 
Table 30: Relationships between some parameters, for $D^{\prime \prime}=2$

\begin{tabular}{cccccccccc}
\hline$D^{\prime \prime}$ & $\nu$ & $D^{\prime \prime}+2 \nu$ & $D$ & $S^{\prime \prime}$ & $\Omega^{\prime \prime}$ & $\sigma^{\prime \prime}$ & $D$ & $D+2 \nu$ & $2 S^{\prime \prime}+1$ \\
\hline 2 & -1 & 0 & $3-\Omega^{\prime \prime}$ & 1 & 1 & +1 & 2 & 0 & 3 \\
2 & -1 & 0 & $3-\Omega^{\prime \prime}$ & 0 & 0 & $\mathrm{NR}$ & 3 & 1 & 1 \\
2 & -1 & 0 & $3-\Omega^{\prime \prime}$ & 1 & -1 & -1 & 4 & 2 & 3 \\
2 & -1 & 0 & $3-\Omega^{\prime \prime}$ & 2 & -4 & -1 & 7 & 5 & 5 \\
2 & -1 & 0 & $3-\Omega^{\prime \prime}$ & 3 & -9 & -1 & 12 & 10 & 7 \\
2 & -1 & 0 & $3-\Omega^{\prime \prime}$ & 4 & -16 & -1 & 19 & 17 & 9 \\
2 & -1 & 0 & $3-\Omega^{\prime \prime}$ & 5 & -25 & -1 & 28 & 26 & 11 \\
2 & -1 & 0 & $3-\Omega^{\prime \prime}$ & 6 & -36 & -1 & 39 & 37 & 13 \\
2 & -1 & 0 & $3-\Omega^{\prime \prime}$ & 7 & -49 & -1 & 52 & 50 & 15 \\
2 & -1 & 0 & $3-\Omega^{\prime \prime}$ & 8 & -64 & -1 & 67 & 65 & 17 \\
2 & -1 & 0 & $3-\Omega^{\prime \prime}$ & 9 & -81 & -1 & 84 & 82 & 19 \\
\hline
\end{tabular}

We suggest that the $0 \mathrm{G} \emptyset$ solution correlates with $\sigma^{\prime \prime}=+1$ and $S^{\prime \prime}=1$. The notion of zero mass pertains.

\subsection{A prediction for the tauon mass}

This unit suggests a relationship, which ongoing theory seems not to discuss, between the ratio of the tauon mass to the electron mass and a ratio of a strength of electromagnetism and the strength of gravity. This unit discusses the notion that adequately increasing the experimental accuracy of either one of the tauon mass and the gravitational constant leads to a prediction regarding the other quantity. This unit discusses aspects, related to G-family physics, that the ratio of forces suggests.

Equation (49) possibly pertains. Here, $m$ denotes mass, $\tau$ denotes tauon, $e$ denotes electron, $q$ denotes charge, $\varepsilon_{0}$ denotes the vacuum permittivity, and $G_{N}$ denotes the gravitational constant. Equation (49) predicts a tauon mass with a standard deviation of less than one quarter of the standard deviation correlating with the experimental result. (For relevant data, see reference [17].)

$$
\begin{gathered}
\beta^{\prime}=m_{\tau} / m_{e} \\
(4 / 3) \times \beta^{12}=\left(\left(q_{e}\right)^{2} /\left(4 \pi \varepsilon_{0}\right)\right) /\left(G_{N}\left(m_{e}\right)^{2}\right) \\
\beta^{\prime}=\beta \\
m_{\tau, \text { calculated }} \approx(1776.8445 \pm 0.024) \mathrm{MeV} / c^{2} \\
m_{\tau, \text { experimental }} \approx(1776.86 \pm 0.12) \mathrm{MeV} / c^{2}
\end{gathered}
$$

The factor of $4 / 3$ in equation (48) correlates with notions that $2 \mathrm{G} 2$ correlates with four so-called channels and 4G4 correlates with three channels. For a $2 \mathrm{G} 2$ interaction between two electrons, the strength for each channel is $\left(\left(q_{e}\right)^{2} /\left(4 \pi \varepsilon_{0}\right)\right) / 4$ and four channels pertain. For a $4 \mathrm{G} 4$ interaction between two electrons, the strength for each channel is $G_{N}\left(m_{e}\right)^{2} / 3$ and three channels pertain.

The following notes pertain.

- To the extent that equation (49) correlates with nature, a more accurate experimental determination of $G_{N}$ or $m_{\tau}$ could predict a more accurate (than experimental results) value for, respectively, $m_{\tau}$ or $G_{N}$.

- Equation (49) links the ratio of two simple particle masses to a ratio of the strengths of two longrange forces.

- Equation (49) links the strength of $2 \mathrm{G} 2$ interactions to the strength of $4 \mathrm{G} 4$ interactions.

- For each $\Sigma \geq 2$ solution that table 17 lists, the number of channels equals the number of blank SA-side cells in an extended version of table 17 that includes the oscillator pair SA9-and-SA10 and embraces the notion that blank (or, $\kappa_{0,-1}$ ) pertains for each added cell. 
- For 6G6, the number of channels is two. For 8G8, the number of channels is one.

- For $\Sigma=10$ and $\Gamma=\Sigma=\llbracket 10 \rrbracket, \Sigma G \Gamma$ would correlate with zero channels and no interactions.

- Equation (52) correlates the fine-structure constant, $\alpha$, with a function of the tauon mass and the electron mass. (Regarding the fine-structure constant, see equation (56).)

$$
\alpha=\left(\left(q_{e}\right)^{2} /\left(4 \pi \varepsilon_{0} \hbar c\right)\right)=(4 / 3) \times\left(m_{\tau} / m_{e}\right)^{12} G_{N}\left(m_{e}\right)^{2} /(\hbar c)
$$

\subsection{The relative strengths of electromagnetism and gravity}

This unit suggests concepts that might correlate with an ongoing theory notion that the strength of gravity is much less than the strength of electromagnetism. This unit suggests a possible relationship between the strength of electromagnetism correlating with monopole interactions with charge and the strength of electromagnetism correlating with dipole interactions with nominal magnetic dipole moment.

For this discussion, we assume that we can work within aspects of proposed theory that de-emphasize translational motion. Below, the symbol if correlates with a non-zero-charge non-zero-mass simple fermion that pertains throughout the discussion. We confine our attention to $1 \mathrm{f} 1 \mathrm{~b} \rightarrow 1 \mathrm{f} 1 \mathrm{~b}$ interactions such that the exiting simple fermion is the same as the entering simple fermion. The simple fermion correlates (as do all simple fermions) with $S=1 / 2$ (or, $\Sigma=1$ ). Regarding modeling, we assume that no translational motion pertains. Hence, no kinematic angular momentum pertains. We assume that conservation of angular momentum pertains. Below, in a symbol of the form $1 \mathrm{f} 1 \mathrm{~b}\left(\Sigma=_{-}\right)$, the expression $\Sigma=$ _ pertains for the boson.

The expression that equation (53) shows can correlate with interactions in which the incoming boson correlates with $2 \mathrm{G} 2$. The interaction flips the spin orientation of the simple fermion. The exiting $1 \mathrm{~b}$ correlates with zero spin. The spin-zero boson might be a OI boson, which has no mass and no charge. (Another possibility might be relevant. The outgoing $1 \mathrm{~b}$ might correlate with a boson ground state. We de-emphasize further discussion of this possibility.) The expression $1 \mathrm{f1b}(\Sigma=2) \rightarrow 1 \mathrm{f} 1 \mathrm{~b}(\Sigma=4)$ can also pertain.

$$
\operatorname{1f1b}(\Sigma=2) \rightarrow \operatorname{1f1b}(\Sigma=0)
$$

We extend our thought experiment to consider $4 \mathrm{G} 4$. The expression $1 \mathrm{f} 1 \mathrm{~b}(\Sigma=4) \rightarrow 1 \mathrm{f} 1 \mathrm{~b}(\Sigma=0)$ does not correlate with interactions. Conservation of angular momentum cannot pertain. The expression $\operatorname{1f1b}(\Sigma=4) \rightarrow \operatorname{lf} 1 \mathrm{~b}(\Sigma=2)$ can pertain. The expression $1 \mathrm{f} 1 \mathrm{~b}(\Sigma=4) \rightarrow 1 \mathrm{f1b}(\Sigma=6)$ can pertain.

The expression $1 \mathrm{f1b}(\Sigma=2) \rightarrow 1 \mathrm{f1b}(\Sigma=0)$ can pertain for each of the following cases $-1 \mathrm{~b}(\Sigma=2)$ correlates with $2 \mathrm{G}, 1 \mathrm{~b}(\Sigma=2)$ correlates with $2 \mathrm{~W}$, and $1 \mathrm{~b}(\Sigma=2)$ correlates (for a case in which $\sigma=-1$ pertains for the $1 \mathrm{f}$ particle) with $2 \mathrm{U}$. This notion might correlate with ongoing theory notions that correlate with relationships between the strengths of the electromagnetic, weak, and strong interactions.

The notion that $\operatorname{1f1b}(\Sigma=4) \rightarrow \operatorname{lf} 1 \mathrm{~b}(\Sigma=0)$ does not pertain for $4 \mathrm{G} 4$ might correlate with ongoing theory notions that the strength of gravity is much less than the strength of electromagnetism.

For each relevant $\Sigma_{1}$, the dominant $1 \mathrm{f} 1 \mathrm{~b}\left(\Sigma_{1}\right) \rightarrow 1 \mathrm{f} 1 \mathrm{~b}\left(\Sigma_{2}\right)$ interaction might comport with the relationship $\Sigma_{1}-2=\Sigma_{2}$. Equation (54) pertains regarding the fine-structure constant $\alpha$. (Compare with equation (56).) We suggest that compatibility exists between the following concepts. One concept is the notion that, in the context of ongoing theory, some modeling might suggest $\alpha^{2} m_{e}$ as the average neutrino mass. (See equation (20).) Regarding proposed theory, the two relevant values of $\Sigma_{1}$ are four and eight. One concept correlates with the ongoing theory notion that terms in anomalous magnetic dipole moment calculations seem to scale with terms proportional to $\alpha^{(\Sigma-2) / 2}$. The concept that $\alpha$ is, in the sense of equation (54), proportional to $\hbar$ might pertain.

$$
\alpha=\left(\left(q_{e} / \hbar\right)^{2} /\left(4 \pi \varepsilon_{0} c\right)\right) \cdot \hbar
$$

Equation (54) might provide a link between the strength of $2 \mathrm{G} 2$ and the strength of $2 \mathrm{G} 24$. The equation includes the term $\left(q_{e} / \hbar\right)^{2}$. The Josephson constant $K_{\mathrm{J}}$ equals $2 q_{e} / \hbar$.

\subsection{The masses of quarks and charged leptons}

This unit shows a formula that links the masses of the six quarks and three charged leptons.

We discuss a formula that approximately fits the masses of the six quarks and three charged leptons. (See equation (55).) The formula includes two integer variables and seven parameters. One integer variable, $M^{\prime \prime}$, correlates somewhat with generation. For the electron and each of the six quarks, the generation equals $M^{\prime \prime}+1$. For each of the muon and the tauon, the generation equals $M^{\prime \prime}$. The other 
Table 31: Approximate rest energies (in $\mathrm{MeV}$ ) for $1 \mathrm{C}$ and $1 \mathrm{Q}$ particles

\begin{tabular}{|c|c|c|c|c|c|}
\hline$M^{\prime \prime}$ & Legend & $\begin{array}{c}M^{\prime} \\
\text { Charge }\end{array}$ & $\begin{array}{c}3 \\
-1 \cdot\left|q_{e}\right|\end{array}$ & $\begin{array}{c}2 \\
+(2 / 3) \cdot\left|q_{e}\right|\end{array}$ & $\begin{array}{c}1 \\
-(1 / 3) \cdot\left|q_{e}\right|\end{array}$ \\
\hline 0 & name & & electron & up & down \\
\hline 0 & data & & $(0.511$ to 0.511$) \times 10^{0}$ & $(1.8$ to 2.7$) \times 10^{0}$ & $(4.4$ to 5.2$) \times 10^{0}$ \\
\hline 0 & calculation & & $m_{e} c^{2} \approx 0.511 \times 10^{0}$ & $m_{u} c^{2} \approx 2.2 \times 10^{0}$ & $m_{d} c^{2} \approx 4.8 \times 10^{0}$ \\
\hline 1 & name & & & charm & strange \\
\hline 1 & data & & & $(1.24$ to 1.30$) \times 10^{3}$ & $(0.92$ to 1.04$) \times 10^{2}$ \\
\hline 1 & calculation & & & $m_{c} c^{2} \approx 1.263 \times 10^{3}$ & $m_{s} c^{2} \approx 0.938 \times 10^{2}$ \\
\hline 2 & name & & muon & top & bottom \\
\hline 2 & data & & $(1.06$ to 1.06$) \times 10^{2}$ & $(1.56$ to 1.74$) \times 10^{5}$ & $(4.15$ to 4.22$) \times 10^{3}$ \\
\hline 2 & calculation & & $m_{\mu} c^{2} \approx 1.06 \times 10^{2}$ & $m_{t} c^{2} \approx 1.72 \times 10^{5}$ & $m_{b} c^{2} \approx 4.18 \times 10^{3}$ \\
\hline 3 & name & & tauon & & \\
\hline 3 & data & & $(1.777$ to 1.777$) \times 10^{3}$ & & \\
\hline 3 & calculation & & $m_{\tau} c^{2} \approx 1.777 \times 10^{3}$ & & \\
\hline
\end{tabular}

integer variable, $M^{\prime}$, correlates with magnitude of charge. The seven parameters can be $m_{e}, m_{\mu}$ (or, the mass of a muon), $\beta, \alpha, d^{\prime}(0), d^{\prime}(1)$, and $d^{\prime}(2)$. Here, $\alpha$ denotes the fine-structure constant. (See equation (56).) Here, $d^{\prime}(k)$ pertains regarding generation- $(k+1)$ quarks. For each generation, the number might correlate with the extent to which the two relevant quark masses do not equal the square root of the multiplicative product of the two quark masses.

Table 31 shows experimental rest energies and calculated rest energies for $1 \mathrm{C}$ and $1 \mathrm{Q}$ simple fermions. Rest energy denotes rest mass times $c^{2}$. The table shows rest energies in units of MeV. (Regarding data from experiments, see reference [17].) For each particle other than the top quark, reference [17] provides one estimate. For the top quark, reference [17] provides three estimates. For each quark, table 31 shows a data range that runs from one standard deviation below the minimum nominal value that reference [17] shows to one standard deviation above the maximum nominal value that reference [17] shows. Each standard deviation correlates with the reported standard deviation that correlates with the nominal value. For charged leptons (that is, for $M^{\prime}=3$ ), the table does not completely specify accuracy regarding ranges. Our calculations use equation (55). In that equation, the factor $3 / 2$ correlates with the average of $M^{\prime}=2$ and $M^{\prime}=1$. (Note the appearance of $M^{\prime}=3 / 2$ in equation (60). The concepts of $M^{\prime}=3 / 2$ and $m\left(M^{\prime \prime}, 3 / 2\right)$ are useful mathematically, though not necessarily directly physics-relevant.) Regarding equations (61), (62), and (63), we choose values that fit data. Regarding each charged lepton, our calculations fit data to more significant figures than the numbers in the table show. Regarding the tauon, our calculation correlates with a mass that may be more accurate, and more accurately specified, than the mass correlating with reference [17] data. (See equations (50) and (51).)

$$
\begin{gathered}
m\left(M^{\prime \prime}, M^{\prime}\right)=m_{e} \times\left(\beta^{1 / 3}\right)^{M^{\prime \prime}+\left(j_{M^{\prime \prime}}^{\prime \prime}\right) d^{\prime \prime}} \times\left(\alpha^{-1 / 4}\right)^{\left(1-\delta\left(M^{\prime}, 3\right)\right) \cdot\left((3 / 2) \cdot\left(1+M^{\prime \prime}\right)+\left(j_{M^{\prime}}^{\prime}\right) d^{\prime}\left(M^{\prime \prime}\right)\right)} \\
\alpha=\left(\left(q_{e}\right)^{2} /\left(4 \pi \varepsilon_{0}\right)\right) /(\hbar c) \\
j_{M^{\prime \prime}}^{\prime \prime}=0,+1,-1,0 \text { for, respectively, } M^{\prime \prime}=0,1,2,3 \\
d^{\prime \prime}=\left(2-\left(\log \left(m_{\mu} / m_{e}\right) / \log \left(\beta^{1 / 3}\right)\right)\right) \approx 3.840679 \times 10^{-2} \\
1-\delta\left(M^{\prime}, 3\right) \text { equals } 0, \text { for } M^{\prime}=3, \text { and equals } 1, \text { otherwise } \\
j_{M^{\prime}}^{\prime}=0,-1,0,+1 \text { for, respectively, } M^{\prime}=3,2,3 / 2,1
\end{gathered}
$$

$$
d^{\prime}(0) \sim 0.318
$$


Table 32: Ranges of $d^{\prime}\left(M^{\prime \prime}\right)$ that fit the data ranges that table 31 shows for quark masses

\begin{tabular}{cccc}
\hline Symbol & $\begin{array}{c}\text { Minimum } \\
\text { (approximate) }\end{array}$ & $\begin{array}{c}\text { Nominal } \\
\text { (table 31) }\end{array}$ & $\begin{array}{c}\text { Maximum } \\
\text { (approximate) }\end{array}$ \\
\hline$d^{\prime}(0)$ & 0.251 & 0.318 & 0.386 \\
$d^{\prime}(1)$ & -1.072 & -1.057 & -1.042 \\
$d^{\prime}(2)$ & -1.5158 & -1.5091 & -1.5024 \\
\hline
\end{tabular}

Table 33: Possible estimates for quark masses

\begin{tabular}{cccccc}
\hline$M^{\prime \prime}$ & Ratio & Value & $d^{\prime}\left(M^{\prime \prime}\right)$ & $m_{M^{\prime}=2}\left(\mathrm{MeV} / c^{2}\right)$ & $m_{M^{\prime}=1}\left(\mathrm{MeV} / c^{2}\right)$ \\
\hline 0 & $m_{e} m_{d} /\left(m_{u}\right)^{2}$ & $1 / 2$ & $\approx 0.31216454$ & $m_{u} \approx 2.203 \times 10^{0}$ & $m_{d} \approx 4.748 \times 10^{0}$ \\
1 & $m(2,3) m_{c} /\left(m_{s}\right)^{2}$ & 1 & -1 & $m_{c} \approx 1.178 \times 10^{3}$ & $m_{s} \approx 1.006 \times 10^{2}$ \\
2 & $m_{\mu} m_{t} /\left(m_{b}\right)^{2}$ & 1 & $-3 / 2$ & $m_{t} \approx 1.695 \times 10^{5}$ & $m_{b} \approx 4.322 \times 10^{3}$ \\
\hline
\end{tabular}

$$
\begin{gathered}
d^{\prime}(1) \sim-1.057 \\
d^{\prime}(2) \sim-1.5091 \\
m(2,3) \approx 8.59341 \mathrm{MeV} / c^{2}
\end{gathered}
$$

Table 32 shows ranges of $d^{\prime}\left(M^{\prime \prime}\right)$ that fit the data ranges that table 31 shows for quark masses. (See equations (61), (62), and (63).) To the extent that people measure quark masses more accurately, people might find relationships between $d^{\prime}(0), d^{\prime}(1)$, and $d^{\prime}(2)$, and thereby reduce the number of parameters to less than seven.

Table 33 shows possible masses for quarks. For each row, we assume the value that the third column shows for the ratio that the second column defines. The value implies the number that the column labeled $d^{\prime}\left(M^{\prime \prime}\right)$ shows. The six estimated quark masses might not be incompatible with experimental results that table 31 shows. To the extent that table 33 comports with nature, fitting the masses of six quarks and three charged leptons requires at most five parameters. The five parameters can be $m_{e}, m_{\mu}, \beta, \alpha$, and $d^{\prime}(0)$. To the extent that table 33 comports with nature, equation (65) pertains.

$$
\left(m_{s}\right)^{2} m_{\mu}=m_{e} m_{\tau} m_{c}
$$

The charge $q_{e}$ correlates with $\beta$, via equation (48). The charge $q_{e}$ appears in $\alpha$, via equation (56). Based on equations (45) and (55) and based on modeling for the G-family, proposed theory might entangle concepts related to mass and concepts related to charge more deeply than does ongoing theory.

\subsection{Neutrino masses}

This unit discusses the notion that all neutrinos might have zero mass, even though people interpret neutrino oscillations and other observed phenomena as suggesting that at least one flavor of neutrino correlates with non-zero mass.

Table 34 lists aspects that might correlate with the extent to which neutrinos have non-zero masses.

We discuss inferences from astrophysics data.

Discussion related to table 21 and to equation (20) suggests modeling that would be compatible with data and with elementary particle Standard Model aspects that suggest that all neutrinos have zero rest masses.

We discuss aspects related to neutrino oscillations.

Ongoing theory hypothesizes that gravity catalyzes neutrino oscillations. This hypothesis might correlate with a process of elimination. Ongoing theory suggests that each known simple particle does not catalyze neutrino oscillations. Ongoing theory suggests that photons do not catalyze neutrino oscillations. The only ongoing theory catalyst for neutrino oscillations would be gravity.

Proposed theory suggests that each of the $4 \mathrm{G} \Gamma$ components of $4 \gamma$ might not correlate with neutrino oscillations. (For one explanation, see table 10a. For another explanation, see table 21 and discussion related to equation (20). Perhaps, note discussion regarding equations (105) and (106).) Solutions 8G2468a and $8 \mathrm{G} 2468 \mathrm{~b}$ correlate with interactions with lepton number. Solutions 8G2468a and 8G2468b do not 
Table 34: Aspects that might correlate with the extent to which neutrinos have non-zero masses

Aspects

- Limits regarding neutrino masses, as inferred from astrophysics data.

- The existence of neutrino oscillations.

- Neutrino speeds.

- Effects of neutrino lensing (which would be based on gravity).

- Other.

correlate with the $S U(2)$ symmetry that correlates with somewhat conservation of fermion generation. (See table 17. In that table, items for which the TA-side symmetry is not $S U(3)$ do not correlate with the relevant $S U(2)$ symmetry.) Proposed theory suggests that interactions correlating with solutions 8G2468a and 8G2468b catalyze neutrino oscillations. Discussion related to equations (114) and (115) offers an explanation, in the context of ongoing theory modeling, for neutrino oscillations. This modeling differs from modeling that we show in discussion related to equation (20). Discussion related to equations (114) and (115) might assume that people can model effects of 8G-induced neutrino oscillations via the notion of, in effect, an index of refraction.

We know of no data about neutrino speeds that would settle the question as to the extent to which neutrinos have non-zero mass.

As far as we know, observations of impacts of possible neutrino lensing have yet to produce relevant results.

As far as we know, other possibly relevant experiments and observations do not provide additional insight about the extent to which neutrinos have non-zero masses. (See, for example, references [31] and [32].)

Proposed theory suggests that each neutrino might correlate with zero rest mass.

\subsection{Anomalous moments}

This unit discusses a proposed theory approach to explaining anomalous magnetic dipole moments.

Equations (66), (67), and (68) show results of experiments regarding anomalous magnetic dipole moments. (See reference [17].) The subscripts $e, \mu$, and $\tau$ denote, respectively, electron, muon, and tauon. The symbol $a$ correlates with anomalous magnetic dipole moment. The symbol $\alpha$ denotes the fine-structure constant.

$$
\begin{gathered}
a_{e}-(\alpha /(2 \pi)) \approx-1.76 \times 10^{-6} \\
a_{\mu}-(\alpha /(2 \pi)) \approx+4.51 \times 10^{-6} \\
-0.052<a_{\tau}<+0.013
\end{gathered}
$$

Ongoing theory provides means, correlating with Feynman diagrams, to calculate an anomalous magnetic dipole moment for each of, at least, the electron and the muon. Regarding the tauon, equation (69) shows a result correlating with a first-order Standard Model (or, ongoing theory) calculation. (See reference [33].)

$$
a_{\tau, \mathrm{SM}} \approx+1.177 \times 10^{-3}
$$

Proposed theory suggests that notions of anomalous electromagnetic moments correlate with $\gamma 2$ solutions. Electromagnetic dipole solutions correlate with $\gamma 2$ solutions for which RSDF is $r^{-3}$. The following remarks pertain for other than the $2 \mathrm{G} 24$ solution, which correlates with the ongoing theory nominal magnetic moment result of $g \approx 2$. (2G24 correlates with $2 \gamma$ and not with $\gamma 2$.) The relevant solutions 
Table 35: Possible approximations regarding the 6G24,1 and 6G24,t contributions to $a_{\mathrm{cl}}-(\alpha /(2 \pi))$ for charged leptons

\begin{tabular}{ccc}
\hline Assumption regarding $t_{\mathrm{cl}}$ & $a_{6 \mathrm{G} 24,1}$ & $a_{6 \mathrm{G} 24, \mathrm{t}}$ \\
\hline$m$ & $-1.79 \times 10^{-6}$ & $5.96 \times 10^{-8}$ \\
$m^{2}$ & $-1.76 \times 10^{-6}$ & $5.62 \times 10^{-10}$ \\
$M^{\prime \prime}$ & $-1.76 \times 10^{-6}$ & $3.13 \times 10^{-6}$ \\
$\left(M^{\prime \prime}\right)^{2}$ & $-1.76 \times 10^{-6}$ & $1.57 \times 10^{-6}$ \\
generation & $-8.03 \times 10^{-6}$ & $6.27 \times 10^{-6}$ \\
$(\text { generation })^{2}$ & $-3.85 \times 10^{-6}$ & $2.09 \times 10^{-6}$ \\
$\log \left(m / m_{e}\right)$ & $-1.76 \times 10^{-6}$ & $1.18 \times 10^{-6}$ \\
$\left(\log \left(m / m_{e}\right)\right)^{2}$ & $-1.76 \times 10^{-6}$ & $2.21 \times 10^{-7}$ \\
\hline
\end{tabular}

Table 36: Possible approximations for $a_{\tau}-(\alpha /(2 \pi))$

\begin{tabular}{ccccc}
\hline $\begin{array}{c}\text { Assumption } \\
\text { regarding first } \\
\text { order behavior } \\
\text { for }\end{array}$ & $\begin{array}{c}\text { First order } \\
\text { suggestion for } \\
a_{\tau}-(\alpha /(2 \pi))\end{array}$ & $\begin{array}{c}\text { Prediction for } \\
a_{\tau}\end{array}$ & $\begin{array}{c}\text { Approximate } \\
\text { comparison } \\
\left(a_{\tau}-a_{\tau, \mathrm{SM}}\right) / a_{\tau, \mathrm{SM}}\end{array}$ & Fit \\
$\begin{array}{c}a_{\mathrm{cl}}-(\alpha /(2 \pi)) . \\
\text { The term is }\end{array}$ & & & \\
linear in a & & & \\
lepton's: & & & & \\
\hline$m$ & $+1.04 \times 10^{2} \times 10^{-6}$ & $+1.266 \times 10^{-3}$ & $+75 \times 10^{-3}$ & - \\
$m^{2}$ & $+1.77 \times 10^{3} \times 10^{-6}$ & $+2.933 \times 10^{-3}$ & $+1500 \times 10^{-3}$ & - \\
$M^{\prime \prime}$ & $+7.65 \times 10^{-6}$ & $+1.169 \times 10^{-3}$ & $-6.9 \times 10^{-3}$ & $!$ \\
$\left(M^{\prime \prime}\right)^{2}$ & $+12.35 \times 10^{-6}$ & $+1.174 \times 10^{-3}$ & $-2.9 \times 10^{-3}$ & $!$ \\
generation & $+10.8 \times 10^{-6}$ & $+1.172 \times 10^{-3}$ & $-4.3 \times 10^{-3}$ & $!$ \\
$(\text { generation })^{2}$ & $+15.0 \times 10^{-6}$ & $+1.176 \times 10^{-3}$ & $-0.7 \times 10^{-3}$ & $! !$ \\
$\log \left(m / m_{e}\right)$ & $+7.83 \times 10^{-6}$ & $+1.169 \times 10^{-3}$ & $-6.8 \times 10^{-3}$ & $!$ \\
$\left(\log \left(m / m_{e}\right)\right)^{2}$ & $+12.9 \times 10^{-6}$ & $+1.174 \times 10^{-3}$ & $-2.5 \times 10^{-3}$ & $!$ \\
\hline
\end{tabular}

might be 4G26, 6G24, 6G28, 8G26, and 10G28. However, 6G28 and 10G28 do not interact with individual simple fermions. These solutions might correlate with, for example, the Lamb shift. Regarding anomalous electromagnetic dipole moments, we assume that 4G26, 6G24, and 8G26 pertain.

Proposed theory suggests that contributions to $a$ scale as $\alpha^{(\Sigma-2) / 2}$. (Compare with discussion leading to equation (20).)

We explore an approach to estimating $a_{\tau}$.

The 4G26 solution might correlate with the ongoing theory result of $\alpha /(2 \pi)$. The $6 \mathrm{G} 24$ solution might correlate with contributions of the order $\alpha^{2}$. People might be able to extrapolate, based on observed strengths of $6 \mathrm{G} 24$, to predict the order $\alpha^{2}$ contribution to $a_{\tau}$.

We assume that, for a charged lepton cl, equation (70) pertains. Here, $t_{\mathrm{cl}}$ is the construct that the first column of table 35 identifies.

$$
a_{\mathrm{cl}}-(\alpha /(2 \pi)) \approx a_{6 \mathrm{G} 24,1}+a_{6 \mathrm{G} 24, \mathrm{t}} t_{\mathrm{cl}}
$$

Table 35 shows approximate possible values for $a_{6 \mathrm{G} 24,1}$ and $a_{6 \mathrm{G} 24, \mathrm{t}}$, based on fitting data that equations (66) and (67) show and using various candidates for $t_{\mathrm{cl}}$. We de-emphasize the notion that $8 \mathrm{G} 26$ might also contribute to an actual value.

Table 36 provides, based on table 35 and equation (70), some possible suggestions for $a_{\tau}-(\alpha /(2 \pi))$. The comparison is with respect to a Standard Model first order calculation. (See equation (69).) Per the notion that the interaction strength does not necessarily correlate linearly or quadratically with an ongoing theory property and per the quadratic behavior with respect to $\left|q_{e}\right|$ in the expression $\alpha^{(\Sigma-2) / 2}$, we might expect that appropriate results might correlate with the square of generation or with the square of a function of $\log (m)$. (See work that includes equation (55).)

Each one of the results that table 36 shows comports with experimental results. Except for the row regarding $m$ and the row regarding $m^{2}$, each row in table 36 might comport with the calculation based on the Standard Model. The (generation) ${ }^{2}$-centric result that table 36 shows might comport best, of the 
results the table suggests, with the calculation based on the Standard Model. The (generation) ${ }^{2}$-centric result differs from the result equation (69) shows by about 0.7 parts in 1000 .

Based on the notion that contributions to $a$ scale as $\alpha^{(\Sigma-2) / 2}$ and on results that table 35 shows, it might seem unlikely that $a_{6 \mathrm{G} 24,1}$ correlates with $8 \mathrm{G} 26$. However, it is possible that the strength of interactions correlating with 4G26 differs from the ongoing theory result that correlates with $\alpha /(2 \pi)$ and that $a_{6 \mathrm{G} 24,1}$ correlates with such a difference.

Given remarks just above, we explore another approach to estimating $a_{\tau}$.

We assume that the strength of each of 4G26 and 8G26 does not change with generation. We assume that, in effect, equation (71) pertains. We assume that, in effect, equation (72) pertains. Here, we have assumed a clean split between contributions that do not correlate with generation and contributions that do correlate with generation. For the left side of equation (71), $4 \notin \Gamma$. For the left side of equation (72), $4 \in \Gamma$. Regarding table 36, the leftmost column and the rightmost three columns pertain regarding this approach. (Technically, one needs to change the column heading for the leftmost column. The new heading should be the following: "Assumption regarding the behavior for $a_{6 \mathrm{G} 24}$. The term is linear in a lepton's:".)

$$
\begin{gathered}
a_{4 \mathrm{G} 26}+a_{8 \mathrm{G} 26}=(\alpha /(2 \pi))+a_{6 \mathrm{G} 24,1} \\
a_{6 \mathrm{G} 24}=a_{6 \mathrm{G} 24, \mathrm{t}}
\end{gathered}
$$

Per the rightmost column in table 36, the property of generation might be an analog for quantumcentric aspects of spin-two G-family physics to the property of charge for quantum-centric aspects of spin-one G-family physics. This notion seems compatible with each of equation (17) and equation (18). In effect, the notion also correlates with the notion that kinematics applications of $4 \gamma$ correlate with properties such as mass or energy. For such kinematics applications, equation (18) pertains and equation (17) does not necessarily pertain.

\subsection{Possible threshold energies for observing effects of tweak (or, 2T) simple particles}

This unit discusses possible masses for tweak simple particles. The unit discusses possible threshold energies for detecting effects correlating with tweaks.

We explore possibilities regarding masses for T-family bosons.

Work above correlates with the notion that the charge of the $\mathrm{T}^{ \pm}$boson is one-third the charge of the $\mathrm{W}$ boson. (See discussion related to table 12.) Aspects regarding charge are additive and correlate with $U(1)$ and $\pi_{0,-1}$ symmetry. None of linear, $U(1)$, and $\pi_{0,-1}$ pertains regarding mass. Presumably, none of linear, $U(1)$, and $\pi_{0,-1}$ pertains regarding squares of mass.

The 0G $\emptyset$ solution correlates with the possible 0I (or, aye) boson. the 0I boson would have zero mass. Zero mass correlates with $\sigma^{\prime \prime}=+1$ and $S^{\prime \prime}=1$. (See, in table 30, the column labeled $D+2 \nu$.) We might try to, in effect, extrapolate from $\sigma^{\prime \prime}=+1$ and $S^{\prime \prime}=1$ for the 0I boson, $\sigma^{\prime \prime}=-1$ and $S^{\prime \prime}=3$ for W-family physics, and $\sigma^{\prime \prime}=-1$ and $S^{\prime \prime}=4$ for H-family physics. The equation $S^{\prime \prime}=7$ would correlate with allowed values of $\lambda$ of two, four, six, eight, 10, 12, and 14 . The equation $S^{\prime \prime}=7$ provides the first possibility (beyond the limit $\lambda \leq 8$ ) to have G-family-like solutions for which $\Sigma=0$. For $S^{\prime \prime}=7$, $D+2 \nu=50$. Proposed theory suggests that equations (73) and (74) might pertain regarding the masses of T-family bosons. Here, we allow for the possibilities of adding or subtracting the integers correlating with $\sigma^{\prime \prime}=+1$ and $S^{\prime \prime}=1, S^{\prime \prime}=0$, and $\sigma^{\prime \prime}=-1$ and $S^{\prime \prime}=1$. Based on data from reference [17] regarding the Higgs boson, the rest energies of the T-family bosons might be between $\sim 208 \mathrm{GeV}$ and $\sim 221 \mathrm{GeV}$.

$$
\begin{aligned}
& 47 / 17 \leq\left(m_{T^{ \pm}}\right)^{2} /\left(m_{H^{0}}\right)^{2} \leq 53 / 17 \\
& 49 / 17 \leq\left(m_{T^{0}}\right)^{2} /\left(m_{H^{0}}\right)^{2} \leq 51 / 17
\end{aligned}
$$

We discuss aspects for experiments that might create and detect effects involving charged $2 \mathrm{~T}$ simple bosons. We assume that detection involves using potentially free-ranging objects as inputs into experiments. Here, we use the word potentially to indicate the possibility that an actual object resides in a larger object. An example would be a proton in an atomic nucleus. We assume that detection involves sensing potentially free-ranging objects that experiments produce. Free-ranging objects have charges that are integer multiples of the magnitude of the charge of an electron. 
We introduce the symbols that equations (75) and (76) show. The symbol $1 \mathrm{Q} \otimes 2 \mathrm{U}$ denotes a particle that includes just quarks and gluons. The word hadron pertains for the particle. The word hadron-like pertains for the particle. Examples of such particles include protons, neutrons, and pions. The symbol $1 \mathrm{R} \otimes 2 \mathrm{U}$ denotes a particle that includes just arcs and gluons. The word hadron-like pertains for the particle. The particle does not include quarks.

$1 \mathrm{Q} \otimes 2 \mathrm{U}$

$1 \mathrm{R} \otimes 2 \mathrm{U}$

The following equations show interactions that convert a neutron into a $1 \mathrm{R} \otimes 2 \mathrm{U}$ hadron-like particle that features three arc (or, 1R) simple fermions. Each symbol $x_{\text {_ }}$ denotes an unspecified simple boson. For each symbol of the form $X^{q}$, the superscript denotes a charge in units of the charge of a positron or proton. Equation (77) shows the creation of two W bosons. Equation (78) shows the use of one of the W bosons to create three tweak (or, 2T) simple bosons. Equation (79) shows the use of the other W boson to convert the up quark in the neutron to a down quark. Equation (80) shows the use of the three tweaks to produce a hadron-like particle that includes three arcs and no quarks. The minimum energy to trigger this set of interactions correlates with the sum of the rest energies of one neutron, one W boson, and three charged tweaks. (A combination of equation (77) and equation (78) correlates with one $\mathrm{W}$ boson and not with two $\mathrm{W}$ bosons.) A range for that minimum energy is $705 \mathrm{GeV}$ to $744 \mathrm{GeV}$.

$$
\begin{array}{r}
x_{1}+x_{2} \rightarrow W^{+1}+W^{-1} \\
x_{3}+x_{4}+W^{+1} \rightarrow 3 T^{+1 / 3} \\
W^{-}+Q^{+2 / 3} \rightarrow Q^{-1 / 3}+x_{5} \\
3\left(T^{+1 / 3}+Q^{-1 / 3} \rightarrow R^{0}+x_{6}\right)
\end{array}
$$

We speculate about means for detecting such a conversion of a neutron into a three-arc hadron-like particle. We assume that the neutron resides in an atomic nucleus in a target material. Given the relevant energies, we assume that the three-arc particle exits the target. We speculate that people would not detect the three-arc particle. The interaction produces one $x_{5}$ boson and three $x_{6}$ bosons. People might detect effects of the $x_{5}$ or $x_{6}$ bosons. People might detect escapes or recoils of atomic nuclei. With one target and enough conversions that do not produce escapes of atomic nuclei, people might detect a change in the isotopic composition of the target.

To convert a proton into a $1 \mathrm{R} \otimes 2 \mathrm{U}$ hadron-like particle that features three arc (or, $1 \mathrm{R}$ ) simple fermions requires one additional instance of equation (79). The minimum energy to trigger the set of interactions correlates with the sum of the rest energies of one proton, two $\mathrm{W}$ bosons, and three charged tweaks. A range for that minimum energy is $786 \mathrm{GeV}$ to $825 \mathrm{GeV}$.

The result for protons might correlate with a finite temperature-dependent half-life for protons.

\subsection{Possibilities for detecting or inferring aye (or, 0I) simple particles}

This unit summarizes bases for possibilities for detecting or inferring the existence of aye simple particles.

Table 37 lists possible roles for the aye particle and for the 0I solution.

We discuss items that table 37 shows.

Discussion related to equation (95) pertains regarding inflation.

Discussion related to equation (96) pertains regarding just after inflation.

Some aspects of ongoing theory propose interactions that would produce unspecified particles that people might not have detected. For example, reference [34] proposes an interaction $K_{L}^{0} \rightarrow \pi^{0}+X$ for which there is an intermediate state of two simple fermions that interact via a $W$ boson and produce the so-designated X particle. (See also [35].) Here, the symbol $K_{L}^{0}$ correlates with the K-Long meson. The symbol $\pi^{0}$ denotes a zero-charge pion. To the extent that this interaction actually occurs, proposed theory suggests the possibility that the $\mathrm{X}$ particle is an aye simple boson.

Ongoing theory proposes concepts such as interactions with a so-called quantum vacuum. Proposed theory does not necessarily embrace a notion of quantum vacuum. Interactions with 0I bosons might produce effects similar to effects that ongoing theory correlates with the notion of interactions with a quantum vacuum.

Discussion related to equation (101) pertains regarding non-zero density of dark energy. 
Table 37: Possible roles for the aye particle and for the 0I solution

(a) Possible roles in nature for the aye particle

Possible roles - the particle ...

Plays a role during the inflationary epoch

Functions as the inflaton and plays a role after inflation

Helps explain some interactions

Explains phenomena that ongoing theory correlates with a so-called quantum vacuum

Explains phenomena that ongoing theory correlates with density of dark energy

Might correlate with situation-specific interaction rates

(b) Possible roles in modeling for the 0I solution

Possible roles - the solution ....

Helps explain scaling by factors of $\alpha$ correlating with adding vertices or with increasing spin

Simplifies some aspects of modeling (and does not necessarily correlate with nature)

Equation (81) shows a possibility for decay of a Higgs boson. The equation might correlate with a rate that is not very situation specific. (Here, we assume a lack of lasing.) Equation (82) shows another possibility for the decay of the Higgs boson. The equation might correlate with a rate that correlates with a density of $0 \mathrm{I}$ particles and might be situation specific.

$$
\begin{gathered}
\mathrm{H}^{0} \rightarrow \ldots \\
\mathrm{OI}+\mathrm{H}^{0} \rightarrow \ldots
\end{gathered}
$$

Discussion related to the relative strengths of some components of long-range forces (or, G-family forces) points to terms proportional to $\alpha^{(\Sigma-2) / 2}$. (See discussion related to equation (53) and discussion related to equation (20).) Possibly, modeling based on the OI solution correlates with aspects regarding spins and interactions. (See, also, discussion related to table 36.)

Table 9a shows a representation for the ground state of the 0I solution. The next two sentences provide possible interpretations. People might interpret the SA-side of the representation as implying that, in nature, the aye particle would not excite. (See table 9a.) People might interpret the SA-side representation as correlating with five channels and, therefore, with the notion that excitement can pertain. (Regarding channels, see discussion regarding equation (48) and discussion regarding equation (121).) Proposed theory suggests that the second possibility pertains. We think that the first possibility might correlate with ongoing theory notions of a quantum vacuum.

\subsection{Relationships between ALG modeling, PDE modeling, kinematics mod- eling, and physics}

This unit summarizes some aspects of ALG modeling and some aspects of PDE modeling. This unit notes constraints that, so far in this essay, pertain regarding modeling. This unit anticipates, later in this essay, relaxing some of the constraints.

We recap aspects of ALG modeling. We recap aspects of PDE modeling. We explore aspects of synergies between ALG modeling and PDE modeling.

ALG techniques and G-family solutions point directly to all long-range forces. ALG techniques and G-family solutions point to all $\sigma=+1$ simple bosons. Absent bounds set by PDE modeling, ALG techniques point to solutions that are not relevant to physics.

PDE techniques assume aspects regarding physics correlating with the G-family and the $2 \mathrm{U}$ subfamily. PDE techniques point to all simple particles, other than $2 \mathrm{U}$ particles, that proposed theory suggests. PDE techniques point to $\sigma=-1$ solutions that might not be relevant to physics.

PDE techniques might not directly point to how ALG techniques can embrace gluons.

A combination of PDE and ALG techniques points to all long-range forces and simple bosons, except gluons, that proposed theory matches or suggests. To some extent, ALG modeling for gluons reflects mainly results from ongoing theory.

ALG modeling expresses property conservation laws. Examples include conservation of charge and conservation of lepton number minus baryon number. ALG modeling suggests approximate conservation 
laws. Examples include approximate conservation of generation and approximate conservation of lepton number.

Modeling regarding kinematics conservation laws has bases in ongoing theory and in ALG modeling.

We discuss modeling that we show above in this essay.

Modeling regarding kinematics features kinematics conservation laws. Modeling de-emphasizes motion. Modeling de-emphasizes specific kinematics models. (Kinematics models can have bases in quantum modeling or classical modeling. Kinematics models can feature aspects of Newtonian physics, special relativity, or general relativity.)

Modeling regarding objects features a notion of a well-defined object, with well-defined internal properties, with well-defined possible interactions with other objects, and with well-defined kinematics properties. (Kinematics properties include energy, momentum, and angular momentum.)

Modeling regarding interactions tends to de-emphasize some aspects of modeling multiple quantum interactions.

We discuss ongoing theory modeling for motions of objects. Modeling recognizes actual interactions between objects. Interactions produce effects. Objects can change internal properties. Objects can change kinematics properties. For example, for a model of a planet orbiting a star, conservation of momentum does not pertain for either of the planet or the star.

We discuss modeling that we show later in this essay. Modeling allows for changes to properties. For example, modeling allows for changes to kinematics properties.

\section{Dark matter, dark energy, astrophysics, and cosmology}

This unit discusses dark matter models that might explain observed ratios of dark matter aspects such as density to ordinary matter aspects such as density. This unit discusses a model that might explain eras regarding the rate of expansion of the universe. This unit discusses models that might explain data about dark energy density of the universe. This unit discusses other astrophysics phenomena and other cosmology phenomena.

\subsection{Models that have bases in one, six, and 36 isomers of charge}

This unit posits that most dark matter correlates with isomers of the charged simple particles. This unit shows models that have bases in one, six, and 36 isomers of charged simple particles. This unit compares features of ongoing theory, PR1ISe modeling, PR6ISe modeling, and PR36ISe modeling.

Work above predicts new simple particles. Work above describes dark energy forces. Work above describes $1 \mathrm{R} \otimes 2 \mathrm{U}$ hadron-like particles. (See discussion regarding equation (76).) The $1 \mathrm{R} \otimes 2 \mathrm{U}$ hadronlike particles contain no charges. The $1 \mathrm{R} \otimes 2 \mathrm{U}$ hadron-like particles do not interact with $2 \gamma$. The $1 \mathrm{R} \otimes 2 \mathrm{U}$ hadron-like particles measure as being dark matter.

Work above does not explain observed ratios of dark matter effects to ordinary matter effects. Some of the ratios correlate with amounts that correlate with gravitational effects. People correlate those effects with the term mass. One of the ratios might correlate with depletion of CMB (or, cosmic microwave background radiation). Ongoing theory seems not to explain these ratios.

Work above correlates with so-called PR1ISe modeling.

The first-known one of the ratios comes from interpretations of measurements of CMB. People infer that the universe includes somewhat more than five times as much dark matter as ordinary matter. People use, regarding the amount for each of dark matter and ordinary matter, the four-word term density of the universe. As far as we know, inferred ratios of density of the universe of dark matter to density of the universe of ordinary matter do not vary much for times that are at least 380 thousand years after the big bang. (Reference [36] indicates a five-plus to one inferred ratio regarding 380 thousand years after the big bang.)

We explore the notion that a five to one ratio reflects something fundamental in nature. We posit that the universe embraces six isomers of charged simple particles. One isomer of basic gravity (or, 4G4) interacts with all of the six isomers of charged simple particles. We say that one isomer of $4 \mathrm{G} 4$ spans six isomers of charged simple particles. Each isomer of charged simple particles correlates with its own isomer of at least two components of $2 \mathrm{G}$ long-range forces. Each isomer of charged particles correlates with its own isomer of 2G2. Each isomer of charged particles correlates with its own isomer of $2 \mathrm{G} 24$. The span for each of $2 \mathrm{G} 2$ and $2 \mathrm{G} 24$ is one.

We use the two-element term PR6ISe modeling to refer to models that embrace the notion that the universe embraces exactly six isomers of charged simple particles. The two letters PR abbreviate the 
Table 38: Cumulative features of various types of modeling

\begin{tabular}{lll}
\hline Modeling & New descriptions and new explanations & New subtleties \\
\hline Ongoing theory & • (Baseline) & \\
PR1ISe & forces simple particles and long-range & • Dark energy forces \\
& - Baryon asymmetry & \\
& - Dark energy density & \\
& - Some dark matter & • Spans \\
PR6ISe or PR36ISe & - More dark matter & \\
& - Ratios of dark matter effects to & \\
& ordinary matter effects & \\
& - Ratios of dark energy density of the & \\
& universe to density of the universe of & \\
& ordinary matter plus dark matter & \\
\hline
\end{tabular}

two-word term physics relevant. The three letters ISe abbreviate the four-word term isomers of the electron.

PR6ISe modeling can explain the five-plus to one ratio of dark matter density of the universe to ordinary matter density of the universe. Five isomers of charged simple particles correlate with dark matter. One isomer of charged simple particles correlates with ordinary matter. The plus in five-plus to one can correlate with $1 \mathrm{R} \otimes 2 \mathrm{U}$ hadron-like particles.

Mathematical modeling correlating with spans suggests that 2G248 has a span of six isomers of charged simple particles.

PR6ISe modeling dovetails with the notion that the span of six for 2G248 embraces the same six isomers of charged simple particles as does the span of six for $4 \mathrm{G} 4$.

PR36ISe modeling embraces the possibility that the span of six for 2G248 is, in effect, orthogonal (or, perpendicular) to the span of six for $4 \mathrm{G} 4$. Here, six isomers of $4 \mathrm{G} 4$ pertain. Each of those six isomers of $4 \mathrm{G} 4$ spans six isomers of charged simple particles. The term doubly-dark matter pertains to the 30 isomers of charged simple particles that do not interact with the ordinary matter isomer of charged particles via 4G4. Doubly dark matter does not interact with ordinary matter via 2G2, 2G24, 4G4, or other components of $4 \mathrm{G}$. Five doubly dark matter isomers of charged simple particles interact with the ordinary matter isomer of charged simple particles via 2G248.

From the perspective of one of the 36 isomers of charged simple particles, the following statements pertain. The isomer correlates with its own isomers of 2G2, 2G24, 4G246, 4G2468a, and 4G2468b. The isomer of charged simple particles interacts via 2G248 with five other isomers of charged simple particles. The isomer of charged simple particles interacts via $4 \mathrm{G} 4$ with five other isomers of charged simple particles. None of the first five other isomers is one of the second five other isomers. The first five other isomers are doubly dark matter isomers. The second five other isomers are dark matter isomers.

We preview features of each of PR1ISe, PR6ISe and PR36ISe modeling.

Table 38 discusses cumulative features of various types of modeling. Generally, each row augments the rows above that row. Regarding PR1ISe, long-range forces include dark energy forces. We think that PR6ISe provides useful insight about nature. Regarding ratios of dark energy density of the universe to density of the universe of ordinary matter plus dark matter, PR36ISe offers an alternative (to PR6ISe) explanation of dark energy density. (See discussion related to equation (101).) Otherwise, regarding bases for aspects that table 38 lists, PR36ISe is similar to PR6ISe. Discussion related to equation (101) suggests that PR6ISe modeling might suffice to explain known phenomena and that it might not be necessary to consider PR36ISe modeling.

\subsection{Spans for objects and long-range forces}

This unit discusses the notion that nature embraces more than one isomer for each of some simple particles, some long-range forces, and some hadron-like particles.

We consider the context of PR6ISe modeling.

We start from the span of six that we posit for 4G4. We consider TA-side symmetries for G-family solutions. (See table 17.) We aim to develop numbers that belong in the table 17 column that has the label span (for $\mathrm{n} \geq 6$ ). The number of generators of each of $S U(3), S U(5)$, and $S U(7)$ divides evenly the integer 48, which is the number of generators of $S U(7)$. Regarding $4 \mathrm{G} 4$, we posit that the expression 
$6=g_{7} / g_{3}$ is relevant. (Regarding notation, see equation (11).) We generalize. We assert that, for each G-family solution for which a symmetry of $S U(j)$ pertains, equation (83) provides the span. We assume that we can generalize from the assumption that the span of $2 \mathrm{G} 2$ is one. For each G-family solution with no TA-side symmetry, the span is one. The W boson has non-zero charge. We assume that the span of the $\mathrm{W}$ boson is one. A span of one comports with information that tables 17 and 20 show. The following sentences pertain. A span of six pertains for the Z boson. A span of one pertains for each of the Higgs boson and 0I boson.

$$
g_{7} / g_{j}
$$

We discuss spans for simple particles and other objects that we do not correlate directly with G-family solutions.

Each charged simple fermion has a span of one. We assume that a span of six pertains for each zero-charge simple fermion. We assume that the spans for $0 \mathrm{~K}$ and $0 \mathrm{P}$ parallel the spans for $0 \mathrm{I}$ and $0 \mathrm{H}$. The span for each of $0 \mathrm{~K}$ and $0 \mathrm{P}$ is one. We assume that the span for $2 \mathrm{U}$ parallels the span for the $\mathrm{Z}$ boson. The span for $2 \mathrm{U}$ is six. The span for $1 \mathrm{Q} \otimes 2 \mathrm{U}$ is one, based on the non-zero charges of $1 \mathrm{Q}$ particles. We assume that the span of $1 \mathrm{R} \otimes 2 \mathrm{U}$ is six.

Equation (84) shows notation for denoting the span, s, for a simple particle or long-range force.

$$
\Sigma(\mathrm{s}) \Phi \text { or } \Sigma(\mathrm{s}) \Phi \Gamma
$$

Ongoing theory does not consider the notion of a span of more than one. Equation (85) characterizes an ongoing theory photon.

$$
2(1) \gamma \text { or } 2(1) \mathrm{G} 2 \oplus 2(1) \mathrm{G} 24 \oplus 2(1) \mathrm{G} 248
$$

For each of each simple particle, each hadron-like particle, and each long-range force, the one-word term span denotes the number of isomers of a set of, at least, non-zero-charge simple particles with which an isomer of the particle or force interacts. The set includes all non-zero-charge simple particles and the ongoing theory photon $2(1) \gamma$.

Table 39 shows the span for each long-range force. The table pertains for each of PR6ISe modeling and PR36ISe modeling. Rows in table 39a list all $\Sigma \gamma$ long-range forces. Rows in table 39b list long-range forces that do not correlate with $\Sigma \gamma$. Table 39 lists 2(6)G248 and does not list 2(1)G248. (See table 39a.)

We consider all three of PR1ISe modeling, PR6ISe modeling, and PR36ISe modeling.

Table 40 summarizes information regarding spans (or equivalently, numbers of isomers) for simple particles, for hadron-like particles, and for some long-range forces. The table summarizes information regarding types of objects with which boson simple particles and some long-range forces interact. The table separates, based on a proposed theory view, elementary particle Standard Model aspects from aspects that the elementary particle Standard Model does not embrace. The magnitude of charge for the $\mathrm{T}^{ \pm}$boson is one-third the magnitude of the charge for each of the $\mathrm{W}^{ \pm}$boson and the electron. The symbol $1 \mathrm{Q} \otimes 2 \mathrm{U}$ correlates with known and possible hadrons. The symbol $1 \mathrm{R} \otimes 2 \mathrm{U}$ correlates with possible hadron-like particles. Regarding the PR36ISe case, the notation $(\| 2 \mathrm{G})$ denotes a span that couples ordinary matter and doubly dark matter. The symbol $\| 2 \mathrm{G}$ correlates with the 3-element phrase parallel to 2G248. Regarding the PR36ISe case, the notation $(\| 4 \mathrm{G})$ denotes a span that couples ordinary matter and dark matter. The symbol $\| 4 \mathrm{G}$ correlates with the 3-element phrase parallel to $4 \mathrm{G} 4$. Regarding the G-family, the table includes just the $\Sigma \gamma$ solutions. Regarding the PR6ISe case, the span for $2 \mathrm{G} 68$ is two. Regarding the PR36ISe case, the span for $2 \mathrm{G} 68$ is two and the notion of $\| 2 \mathrm{G}$ pertains. Table 40 shows the extent to which each of the simple bosons and some of the long-range forces interacts directly with each of at least some simple fermions and with each of at least some multicomponent objects. The symbol Y denotes that interactions occur. The symbol ${ }^{\dagger}$ denotes that somewhat conservation of fermion generation pertains for $1 \mathrm{f} 1 \mathrm{~b} \rightarrow 1 \mathrm{f} 1 \mathrm{~b}$ interaction vertices. The symbol $\mathrm{N}$ denotes that interactions do not occur. Proposed theory suggests the possibility that neither the $0 \mathrm{H}$ boson nor the 0I boson interacts directly with multicomponent objects. Proposed theory suggests that G-family solutions for which the TA-side symmetry is $S U(5)$ do not correlate with direct interactions with simple fermions. (See discussion related to table 14 and discussion related to table 17.) Proposed theory suggests that the G-family solution for which the TA-side symmetry is $S U(7)$ does not correlate with interactions with simple fermions and does not correlate with interactions with multicomponent objects, except for interactions that change only the internal entropy of multicomponent objects. (See discussion related to equation (133).) For each simple boson for which $\sigma=-1$ and table 40 shows a non-one span, the non-one span numbers result from 
Table 39: A catalog of long-range forces

(a) Long-range forces for which $\Sigma \in \Gamma$

\begin{tabular}{cccccc}
\hline $\begin{array}{c}\Sigma \Gamma \\
(\Sigma \in \Gamma)\end{array}$ & $S$ & $\begin{array}{c}\text { Monopole } \\
\left(\mathrm{RSDF}=r^{-2}\right)\end{array}$ & $\begin{array}{c}\text { Dipole } \\
\left(\mathrm{RSDF}=r^{-3}\right)\end{array}$ & $\begin{array}{c}\text { Quadrupole } \\
\left(\mathrm{RSDF}=r^{-4}\right)\end{array}$ & $\begin{array}{c}\text { Octupole } \\
\left(\mathrm{RSDF}=r^{-5}\right)\end{array}$ \\
\hline Yes & 1 & $2(1) \mathrm{G} 2$ & $2(1) \mathrm{G} 24$ & $2(6) \mathrm{G} 248$ & \\
Yes & 2 & $4(6) \mathrm{G} 4$ & $4(2) \mathrm{G} 48$ & $4(1) \mathrm{G} 246$ & $4(1) \mathrm{G} 2468 \mathrm{a}$ \\
Yes & 2 & & & & $4(1) \mathrm{G} 2468 \mathrm{~b}$ \\
Yes & 3 & $6(2) \mathrm{G} 6$ & & & \\
Yes & 4 & $8(1) \mathrm{G} 8$ & & & $8(1) \mathrm{G} 2468 \mathrm{a} 468$ \\
Yes & 4 & & & $8(1) \mathrm{G} 2468 \mathrm{~b}$ \\
\hline
\end{tabular}

(b) Long-range forces for which $\Sigma \notin \Gamma$

\begin{tabular}{|c|c|c|c|c|c|}
\hline $\begin{array}{c}\Sigma \Gamma \\
(\Sigma \in \Gamma)\end{array}$ & $S$ & $\begin{array}{c}\text { Monopole } \\
\left(\mathrm{RSDF}=r^{-2}\right)\end{array}$ & $\begin{array}{c}\text { Dipole } \\
\left(\mathrm{RSDF}=r^{-3}\right)\end{array}$ & $\begin{array}{c}\text { Quadrupole } \\
\left(\mathrm{RSDF}=r^{-4}\right)\end{array}$ & $\begin{array}{c}\text { Octupole } \\
\left(\mathrm{RSDF}=r^{-5}\right)\end{array}$ \\
\hline No & 1 & & $2(6) \mathrm{G} 46$ & $2(6) \mathrm{G} 468$ & \\
\hline No & 1 & & $2(2) \mathrm{G} 68$ & & \\
\hline No & 2 & & $4(6) \mathrm{G} 26$ & $4(6) \mathrm{G} 268$ & \\
\hline No & 3 & & $6(1) \mathrm{G} 24$ & $6(6) \mathrm{G} 248$ & \\
\hline No & 3 & & $6(2) \mathrm{G} 28$ & & \\
\hline No & 4 & & $8(6) \mathrm{G} 26$ & $8(1) \mathrm{G} 246$ & \\
\hline No & 5 & & $10(2) \mathrm{G} 28$ & $10(6) \mathrm{G} 248$ & \\
\hline No & 5 & & $10(6) \mathrm{G} 46$ & $10(6) \mathrm{G} 468$ & \\
\hline No & 6 & & $12(2) \mathrm{G} 48$ & $12(1) \mathrm{G} 246$ & $12(1) \mathrm{G} 2468$ \\
\hline No & 6 & & & $12(6) \mathrm{G} 268$ & \\
\hline No & 7 & & 14(2)G68 & 14(6)G248 & \\
\hline No & 8 & & & $16(6) \mathrm{G} 268$ & $16(1) \mathrm{G} 2468$ \\
\hline No & 9 & & & 18(6)G468 & \\
\hline No & 10 & & & & $20(1) \mathrm{G} 2468$ \\
\hline
\end{tabular}


Table 40: Particles and solutions that correlate with one isomer and particles and solutions that might correlate with more than one isomer; plus, the extent to which simple bosons and some long-range forces interact with simple fermions and with multicomponent objects (with the symbol MCO denoting multicomponent object; with the symbol ${ }^{\dagger}$ denoting that somewhat conservation of fermion generation pertains for $1 \mathrm{f} 1 \mathrm{~b} \rightarrow 1 \mathrm{f} 1 \mathrm{~b}$ interaction vertices)

\begin{tabular}{ccccccc}
\hline $\begin{array}{c}\text { Standard Model } \\
\text { entities }\end{array}$ & $\begin{array}{c}\text { Possible } \\
\text { entities }\end{array}$ & $\begin{array}{c}\text { PR1ISe } \\
\text { span }\end{array}$ & $\begin{array}{c}\text { PR6ISe } \\
\text { span }\end{array}$ & $\begin{array}{c}\text { PR36ISe } \\
\text { span }\end{array}$ & $\begin{array}{c}\text { 1b interact } \\
\text { w/ 1f }\end{array}$ & $\begin{array}{c}\text { 1b interact } \\
\text { w/ MCO }\end{array}$ \\
\hline $1 \mathrm{C}(\sigma=+1)$ & - & 1 & 1 & 1 & - & - \\
$1 \mathrm{~N}(\sigma=+1)$ & - & 1 & 6 & 36 & - & - \\
$1 \mathrm{Q}(\sigma=-1)$ & - & 1 & 1 & 1 & - & - \\
- & $1 \mathrm{R}(\sigma=-1)$ & 1 & 6 & 36 & - & - \\
$2 \mathrm{U}(\sigma=-1)$ & - & 1 & 6 & 36 & $\mathrm{Y}^{\dagger}$ & $\mathrm{N}$ \\
$2 \mathrm{~W}: \mathrm{Z}(\sigma=+1)$ & & 1 & 6 & $6(\| 2 \mathrm{G})$ & $\mathrm{Y}^{\dagger}$ & $\mathrm{N}$ \\
& $2 \mathrm{~T}: 2 \mathrm{~T}^{0}(\sigma=-1)$ & 1 & 6 & $6(\| 2 \mathrm{G})$ & $\mathrm{Y}^{\dagger}$ & $\mathrm{N}$ \\
$2 \mathrm{~W}: \mathrm{W}^{ \pm}(\sigma=+1)$ & $2 \mathrm{~T}: 2 \mathrm{~T}^{ \pm}(\sigma=-1)$ & 1 & 1 & 1 & $\mathrm{Y}^{\dagger}$ & $\mathrm{N}$ \\
$1 \mathrm{Q} \otimes 2 \mathrm{U}(\sigma=+1)$ & - & 1 & 1 & 1 & - & - \\
- & $1 \mathrm{R} \otimes 2 \mathrm{U}(\sigma=+1)$ & 1 & 6 & 36 & - & - \\
$0 \mathrm{H}(\sigma=+1)$ & - & 1 & 1 & 1 & $\mathrm{Y}$ & $\mathrm{N}$ \\
- & $0 \mathrm{P}(\sigma=-1)$ & 1 & 1 & 1 & $\mathrm{~N}$ & $\mathrm{Y}$ \\
- & $0 \mathrm{I}(\sigma=+1)$ & 1 & 1 & 1 & $\mathrm{Y}$ & $\mathrm{N}$ \\
- & $0 \mathrm{~K}(\sigma=-1)$ & 1 & 1 & 1 & $\mathrm{~N}$ & $\mathrm{Y}$ \\
$2 \mathrm{G} 2(\sigma=+1)$ & - & 1 & 1 & 1 & $\mathrm{Y}$ & $\mathrm{Y}$ \\
$2 \mathrm{G} 24(\sigma=+1)$ & - & 1 & 1 & 1 & $\mathrm{Y}$ & $\mathrm{Y}$ \\
$2 \mathrm{G} 248(\sigma=+1)$ & - & 1 & 6 & $6(\| 2 \mathrm{G})$ & $\mathrm{Y} \dagger$ & $\mathrm{Y}$ \\
- & $4 \mathrm{G} 4(\sigma=+1)$ & 1 & 6 & $6(\| 4 \mathrm{G})$ & $\mathrm{Y} \dagger$ & $\mathrm{Y}$ \\
- & $4 \mathrm{G} 48(\sigma=+1)$ & 1 & 2 & $2(\| 4 \mathrm{G})$ & $\mathrm{N}$ & $\mathrm{Y}$ \\
- & $4 \mathrm{G} 246(\sigma=+1)$ & 1 & 1 & 1 & $\mathrm{Y}$ & $\mathrm{Y}$ \\
- & $4 \mathrm{G} 2468 \mathrm{a}(\sigma=+1)$ & 1 & 1 & 1 & $\mathrm{Y}$ & $\mathrm{Y}$ \\
- & $4 \mathrm{G} 2468 \mathrm{~b}(\sigma=+1)$ & 1 & 1 & 1 & $\mathrm{Y}$ & $\mathrm{Y}$ \\
- & $6 \mathrm{G} 6(\sigma=+1)$ & 1 & 1 & 1 & $\mathrm{~N}$ & $\mathrm{Y}$ \\
- & $6 \mathrm{G} 468(\sigma=+1)$ & 1 & 1 & 1 & $\mathrm{Y}$ & $\mathrm{Y}$ \\
- & $8 \mathrm{G} 8(\sigma=+1)$ & 1 & 1 & 1 & $\mathrm{~N}$ & $\approx \mathrm{N}$ \\
- & $8 \mathrm{G} 2468 \mathrm{a}(\sigma=+1)$ & 1 & 1 & 1 & $\mathrm{Y}$ & $\mathrm{Y}$ \\
- & $8 \mathrm{G} 2468 \mathrm{~b}(\sigma=+1)$ & 1 & 1 & 1 & $\mathrm{Y}$ & $\mathrm{Y}$ \\
\hline
\end{tabular}

mathematics. The effective span depends on the span correlating with the object (such as a hadron-like object) in which the simple boson exists.

In table 40 , the items for which $\sigma=+1$ and the PR36ISe span is 36 are $1 \mathrm{~N}$ and $1 \mathrm{R} \otimes 2 \mathrm{U}$. The $1 \mathrm{~N}$ simple particles (or, neutrinos) have zerolike mass and zero charge. For $1 \mathrm{R} \otimes 2 \mathrm{U}$, the component simple particles have zerolike or zero mass and zero charge.

No simple fermion has a zero lepton number minus baryon number. That statement might correlate with a lack of relevance for would-be PR216ISe models. Here, 216 equals $6^{3}$.

We discuss concepts regarding the 2(2)G68 solution.

The 2(2)G68 solution does not belong to the set of $2 \gamma$ solutions and does not belong to the set of $\gamma 2$ solutions. The 2(2)G68 solution does not correlate with interactions with individual simple fermions. Table 21 correlates $\lambda=6$ with baryons and $\lambda=8$ with leptons. We posit that 2(2)G68 correlates with some electromagnetic (or, $\Sigma=2$ ) interactions with atoms and other objects that include both baryons and leptons.

Each of 2(1)G2 and 2(1)G24 correlates with some electromagnetic (or, $\Sigma=2$ ) interactions with atoms and other objects that include both baryons and leptons.

Unlike for the cases of electromagnetic interactions that correlate with 2(1)G2 and 2(1)G24, 2G produced by ordinary matter objects interacts with dark matter objects (for the case in which PR06ISe pertains to nature) or doubly dark matter objects (for the case in which PR36ISe pertains to nature) via $2(2)$ G68. Unlike for the cases of electromagnetic interactions that correlate with 2(1)G2 and 2(1)G24, $2 \mathrm{G}$ produced by some dark matter objects (for the case in which PR06ISe pertains to nature) or by some doubly dark matter objects (for the case in which PR36ISe pertains to nature) interacts with ordinary matter via $2(2) \mathrm{G} 68$. 
We note that discussion above de-emphasizes some aspects that correlate with PR6ISe modeling and PR36ISe modeling.

For the case of PR6ISe, one can label the isomers of charged particles by using the numbers zero through five. Perhaps, one would choose the number zero to pertain to the ordinary matter isomer. One can choose the labels such that the next three sentences pertain. One instance of 4(2)G48 connects isomers zero and one. One instance of 4(2)G48 connects isomers two and three. One instance of 4(2)G48 connects isomers four and five. The issue of the isomers that 2(2)G68 connects remains open. For example, 2(2)G68 connections might match the pairings that pertain for 4(2)G48. Or, for example, for 2(2)G68 the pairings five and zero, one and two, and three and four might pertain. This essay de-emphasizes the issue of which 2(2) G68 pairings pertain.

Other long-range forces components have spans of two. (See table 39.) Similar PR6ISe modeling choices regarding alignment regarding spans pertain regarding these components. This essay deemphasizes the issue of which pairings (relative at least to the 4(2)G48 pairs and maybe also to other pairings, such as 2(2)G68 pairings) pertain.

For the case of PR36ISe, various alignment choices pertain. This essay assumes that each 2(6)G connection is orthogonal to each 4(6)G $\mathrm{G}$ connection. (Here, regarding notation, the $\Gamma$ in $2(6) \mathrm{G} \Gamma$ does not match the $\Gamma$ in $4(6) \mathrm{G} \Gamma$.) Possibly, such an assumption need not pertain. Issues, similar to PR6ISe issues for components with span two, might pertain regarding components with span two. This essay de-emphasizes such issues.

\subsection{Densities of the universe}

This unit discusses various densities of the universe. This unit explores numerical relationships that data suggests.

Ongoing theory discusses five partial densities of the universe. The symbol $\Omega_{\nu}$ denotes neutrino density of the universe. The symbol $\Omega_{\gamma}$ denotes photon density of the universe. The symbol $\Omega_{\mathrm{b}}$ denotes ordinary matter (or, baryonic matter) density of the universe. The symbol $\Omega_{\mathrm{c}}$ denotes dark matter (or, cold dark matter) density of the universe. The symbol $\Omega_{\Lambda}$ denotes dark energy density of the universe. Each of the five densities correlates with data. Equation (86) pertains regarding the total density of the universe, $\Omega$.

$$
\Omega=\Omega_{\nu}+\Omega_{\mathrm{c}}+\Omega_{\mathrm{b}}+\Omega_{\gamma}+\Omega_{\Lambda}
$$

Proposed theory suggests equation (87). The symbol $\Omega_{\mathrm{h}}$ denotes $1 \mathrm{R} \otimes 2 \mathrm{U}$ density of the universe. The symbol $\Omega_{\mathrm{ib}}$ denotes dark matter baryonic density of the universe. (The letter i symbolizes the word isomer.) The symbol $\Omega_{\mathrm{i} \gamma}$ denotes dark matter photon density of the universe.

$$
\Omega_{\mathrm{c}}=\Omega_{\mathrm{h}}+\Omega_{\mathrm{ib}}+\Omega_{\mathrm{i} \gamma}
$$

We interpret data regarding recent states of $\mathrm{CMB}$ (or, cosmic microwave background radiation) as correlating with equation (88). The symbol $\Omega_{\mathrm{h}}$ correlates with the plus in the ratio five-plus to one. The relationship $\Omega_{\mathrm{b}} \gg \Omega_{\gamma}$ pertains regarding data. (Reference [17] provides data.)

$$
\Omega_{\mathrm{ib}} \approx \Omega_{\mathrm{ib}}+\Omega_{\mathrm{i} \gamma}=5\left(\Omega_{\mathrm{b}}+\Omega_{\gamma}\right) \approx 5 \Omega_{\mathrm{b}}
$$

Equation (92) estimates $\Omega_{\mathrm{ib}}$ for the current state of the universe. (Reference [17] provides the data that equations (89), (90), and (91) show.)

$$
\begin{gathered}
\Omega_{\mathrm{b}} \approx 0.0484 \pm 0.001 \\
\Omega_{\mathrm{c}} \approx 0.258 \pm 0.011 \\
\Omega_{\gamma} \approx 0.0000538 \pm 0.0000150 \\
\Omega_{\mathrm{ib}} \approx \Omega_{\mathrm{c}}-5 \Omega_{\mathrm{b}} \approx 0.016
\end{gathered}
$$

Reasons exist for not taking the results that equation (92) shows to be exact. For example, we note the size of the standard deviation in equation (90). 
We interpret data regarding CMB about 380 thousand years after the big bang as correlating with equation (93). (Reference [36] provides data.) Regarding this data, the relationship $\Omega_{\mathrm{b}}<\Omega_{\gamma}$ pertains. We assume that, at 380 thousand years after the big bang, (indirect) effects of dark matter photons on ordinary matter CMB had yet fully to manifest themselves.

$$
\Omega_{\mathrm{ib}}+\Omega_{\mathrm{h}} \approx 5 \Omega_{\mathrm{b}}
$$

\subsection{Dark matter ratios inferred from data regarding cosmic microwave back- ground radiation}

This unit discusses dark matter ratios that people infer from data about cosmic microwave background radiation.

We know of up to two types of CMB (or, cosmic microwave background radiation) observations that might measure ratios of dark matter effects to ordinary matter effects.

One type of observation measures ratios of dark matter density of the universe to ordinary matter density of the universe. (See discussion that leads to table 38 and includes equation (83).) A ratio of five-plus to one seems to pertain for billions of years. (See discussion related to equations (88) and (93).). We use that ratio to posit the basis for PR6ISe modeling. The basis features the notion of six isomers of non-zero-charge simple particles.

The other type of observation might also measure ratios of dark matter effects to ordinary matter effects related to CMB. People measure absorption of CMB via hyperfine interactions with hydrogen-like atoms. (See reference [24].) The amount of absorption is twice or somewhat more than twice the amount that people expected. At least one person speculates that the amount above expectations correlates with effects of dark matter. (See reference [37].)

Proposed theory suggests the following explanation. Solution 2(2)G68 has a span of two. 2(2)G68 interactions are 2(2)G interactions. Equation (94) pertains. Solution 2(2)G68 does not correlate with interactions with individual simple fermions. (The TA-side symmetry is $S U(5)$. See table 17.) Solution 2(2)G68 might correlate with hyperfine interactions. (Note, for example, that the six in $\Gamma$ might correlate with aspects of baryons. The eight in $\Gamma$ might correlate with at least one of lepton number and spin.) Half or somewhat less than half of the observed absorption correlates with the ordinary matter isomer of hydrogen atoms. An equal amount of the observed effect correlates with hydrogen-atom isomers that correlate with one dark matter isomer of PR6ISe-span-one phenomena or with one doubly dark matter isomer of PR6ISe-span-one phenomena. The dark matter case correlates with PR6ISe modeling. The doubly dark matter case correlates with PR36ISe modeling. To the extent that the ordinary matter absorption is less than half of the total absorption, other $2 \mathrm{G} \Gamma$ solutions with spans of at least two might correlate with relevant effects. Each one of solutions 2(6)G46 and 2(6)G468 might pertain. Each of six and eight appears in both the $\Gamma$ for 2(2)G68 and the $\Gamma$ for 2(6)G468. Solution 2(6)G468 correlates with a quadrupole effect, whereas solution 2(2)G68 correlates with a dipole effect.

$$
2 \mathrm{G} 68 \notin 2 \gamma, 2 \mathrm{G} 68 \notin \gamma 2
$$

\subsection{The rate of expansion of the universe}

This unit discusses dark energy forces and suggests an explanation for eras regarding the rate of expansion of the universe.

Two thought experiments set the stage for discussing aspects regarding the rate of expansion of the universe.

We consider one thought experiment. We consider two similar neighboring clumps of stuff. We assume that the clumps are moving away from each other. We assume that the clumps will continue to move away from each other. We assume that, initially, interactions correlating with RSDF $r^{-(n+1)}$ dominate regarding interactions between the two clumps. We assume that the two clumps interact via interactions correlating with RSDF $r^{-n}$. We assume that no other forces have adequate relevance. We assume that the distance between the objects increases adequately. Eventually, the RSDF $r^{-n}$ force dominates the RSDF $r^{-(n+1)}$ force.

We consider a similar thought experiment. We consider two similar neighboring clumps. We assume that these clumps are less interactive (for example, less massive) than the two clumps in the first thought experiment. Generally, dominance of the RSDF $r^{-n}$ force over the RSDF $r^{-(n+1)}$ force occurs sooner 
Table 41: Eras and 4G forces, regarding expansion of the universe

\begin{tabular}{cccccc}
\hline Era & $\mathrm{A} / \mathrm{R}$ & $\mathrm{RSDF}$ & $\begin{array}{c}\text { Components } \\
\text { of } 4 \gamma\end{array}$ & $\begin{array}{c}\text { Other } \\
\text { components } \\
\text { of 4G }\end{array}$ & $\begin{array}{c}\text { Span } \\
\text { (PR6ISe or } \\
\text { PR36ISe) }\end{array}$ \\
\hline early acceleration & net repulsive & $r^{-5}$ & $\begin{array}{c}4(1) \mathrm{G} 2468 \mathrm{a}, \\
4(1) \mathrm{G} 2468 \mathrm{~b}\end{array}$ & & 1 \\
deceleration & net attractive & $r^{-4}$ & $4(1) \mathrm{G} 246$ & $4(1) \mathrm{G} 268$ & 1 \\
$\begin{array}{c}\text { recent acceleration } \\
\text { (recent, for smaller } \\
\text { objects) }\end{array}$ & net repulsive & $r^{-3}$ & $4(2) \mathrm{G} 48$ & $4(2) \mathrm{G} 26$ & 2 \\
& attractive & $r^{-2}$ & $4(6) \mathrm{G} 4$ & & 6 \\
\hline
\end{tabular}

for the two clumps in the second thought experiment that it does for the two clumps in the first thought experiment.

Table 41 summarizes, regarding the rate of expansion of the universe, eras and $4 \mathrm{G}$ forces. In this context, the eras pertain to the largest objects that people can directly infer. Early acceleration pertains for some time after the big bang. Then, deceleration pertains for some billions of years. (Regarding observations that correlate with the eras that correlate with deceleration and recent acceleration, see references [9], [10], [11], and [12].) Acceleration pertains for the most recent few billion years. Regarding smaller objects, dominant forces within objects and between neighboring objects have, at least conceptually, generally transited parallels to the above-mentioned eras and now generally exhibit behavior correlating with RSDF of $r^{-2}$. Quasar formation via ejection of stuff from near or inside black holes might constitute an exception. Black hole jets might constitute an exception. Blazars might constitute an exception. Eruptions from supermassive black holes might constitute an exception. (Regarding such eruptions, see references [38] and [39].) For these cases, $r^{-3}$ net repulsion might pertain. The column labeled A/R notes net effects, across forces dominating for each era. The column labeled components of $4 \gamma$ lists solutions that might correlate with significant forces. (See table 18.) Proposed theory suggests that, for the components of $4 \gamma$ that table 41 lists, the two-word term net repulsive correlates with a notion of essentially always repulsive (though perhaps sometimes not significantly repulsive). Proposed theory suggests that, for the components of $4 \gamma$ that table 41 lists, the two-word term net attractive correlates with a notion of essentially always attractive (though perhaps sometimes not significantly attractive).

Proposed theory suggests that the ongoing theory notion of dark energy forces (or, dark energy pressure) correlates with the components, other than 4(6)G4, of $4 \gamma$.

A better characterization than the six-word term rate of expansion of the universe might feature a notion of the rates of moving apart of observed very large astrophysical objects.

\subsection{Phenomena during and just after inflation}

This unit discusses phenomena that might correlate with times during and just after an inflationary epoch.

Ongoing theory suggests that an inflationary epoch might have occurred. Ongoing theory suggests that the epoch ended around $10^{-33}$ seconds to $10^{-32}$ seconds after the big bang. We are not certain as to the extent that data confirms the occurrence of an inflationary epoch.

Ongoing theory includes models that people claim would support notions of inflation. The models point to states of the universe, at and somewhat after the inflationary epoch, that would provide bases for evolution that would be consistent with observations about later phenomena and would be consistent with aspects of ongoing theory. (Reference [40] summarizes aspects related to inflation, points to references regarding ongoing theory, and discusses some ongoing theory work.)

Reference [13] suggests the possibility that a repulsive aspect of gravity drove phenomena correlating with the inflationary epoch. The reference suggests that the composition of the universe was nearly uniform spatially. The reference suggests the importance of a so-called inflaton field.

Proposed theory suggests the possibility that, during the inflationary epoch, aye particles (or, 0I particles) provided a major non-long-range force component of the universe. The aye particle matches ongoing theory notions of a boson with zero spin. (See reference [40].) Ongoing theory uses the word inflaton to name that boson. Proposed theory suggests the possibility that the octupole components of $4 \gamma$ provided the repulsive aspect of gravity. (See, for example, table 43.) Those components interact with individual simple particles and are repulsive. Equation (95) shows such an interaction. Here, kinematics modeling pertains. (See, as a parallel, equation (106).) Here quantum transitions for 0I particles do not 
necessarily pertain. (See, as a parallel equation (105).) Here, $\mathrm{x}$ and $\mathrm{y}$ might be either of a and b. The end of the inflationary epoch would correlate with a change, regarding effects of $4 \gamma$, from dominance of octupole repulsion to dominance by quadrupole attraction. The end of the inflationary epoch might also correlate with a growth of spatial inhomogeneities regarding (at least) aye particles. The quadrupole component of $4 \gamma$ might help catalyze some of the spatial inhomogeneities. The quadrupole component of $4 \gamma$ might amplify some of the spatial inhomogeneities.

$$
0 \mathrm{I}+4(1) \mathrm{G} 2468 \mathrm{x} \rightarrow 0 \mathrm{I}+4(1) \mathrm{G} 2468 \mathrm{y}
$$

References [13] and [40] suggest that inflaton particles dominated the non-long-range-force composition of the universe for some time after the inflationary epoch. Inflatons produced a cascade of interactions that led to a preponderance of protons, neutrons, and electrons. Clumping of the resulting hydrogen atoms led to the formation of stars.

Proposed theory suggests the possibility that, for some time just after the inflationary epoch, the aye particle might have been a dominant non-long-range-force component of the universe. The dominant longrange force would have been the quadrupole component of $4 \gamma$. That component interacts with individual simple particles and is attractive. Interactions between aye particles would produce components of $2 \gamma$ long-range forces. (See equation (96).) Interactions between aye particles would produce non-aye simple bosons. From there, the above-mentioned cascade could occur. Proposed theory suggests the possibility that quadrupole $4 \gamma$ attraction contributed to clumping.

$$
0 \mathrm{I}+0 \mathrm{I} \rightarrow 2 \gamma+2 \gamma
$$

Discussion above de-emphasizes the question of the extent to which, for clumps or objects that involve multiple simple particles, $4 \gamma$ octupole repulsion might dominate $4 \gamma$ quadruple attraction for at least some time after the end of the inflationary epoch.

Discussion above de-emphasizes the notions of isomers and spans. Discussion above de-emphasizes the notion of phenomena that might have preceded the inflationary epoch.

We discuss isomers and spans.

Our work considers three PRnISe cases $-\mathrm{n}$ is one, $\mathrm{n}$ is six, and $\mathrm{n}$ is 36 . Table 40 suggests that the span for each of the aye particle, the quadrupole component of $4 \gamma$, and the two octupole components of $4 \gamma$ is one. For each one of the PR6ISe case and the PR36ISe case, proposed theory might point to the notion that each of the $\mathrm{n}$ isomers develops similarly to and originally essentially independently from the other ( $\mathrm{n}$ minus one) isomers. Coupling between isomers might start with the production of simple particles that have spans that exceed one. Coupling might also involve, for example, contributions correlating with the $4 \mathrm{G} 48$ component of $4 \gamma$, the $4 \mathrm{G} 4$ component of $4 \gamma$, and the $2 \mathrm{G} 248$ component of $2 \gamma$.

We mention the notion that, for each PRnISe case, the span of the aye particle might be n. For the cases of $\mathrm{n}$ being six and $\mathrm{n}$ being 36, this notion might correlate with the development of roughly equal amounts of each isomer. The involvement of $4 \mathrm{G} 2468 \mathrm{x}, 4 \mathrm{G} 246$, and simple bosons having spans of one might contribute to the rough equality. We do not discuss this notion further.

\subsection{Baryon asymmetry}

This unit discusses proposed theory explanations for baryon asymmetry.

To the extent that the early universe featured roughly the same number of antimatter quarks as matter quarks, something happened to create baryon asymmetry. The two-word term baryon asymmetry correlates with the present lack, compared to matter quarks, of antimatter quarks.

Proposed theory suggests scenarios that might have led to baryon asymmetry. None of the scenarios conserves baryon number. Each scenario conserves lepton number minus baryon number.

In one scenario, the $2 \mathrm{~T}^{ \pm}$boson converts antimatter quarks to matter quarks. This scenario depends on the physics-relevance of $1 \mathrm{R}$ simple fermions. Equation (97) shows an example of a $1 \mathrm{f} 1 \mathrm{~b} \rightarrow 1 \mathrm{f} 1 \mathrm{~b}$ interaction. (Per remarks above, interactions of the form $1 \mathrm{f} 0 \mathrm{~b} \rightarrow 1 \mathrm{f} 1 \mathrm{~b}$ correlate with $1 \mathrm{f} 1 \mathrm{~b} \rightarrow 1 \mathrm{f} 1 \mathrm{~b}$.) Here, the superscripts correlate with charge, in units of $\left|q_{e}\right|$. The subscripts correlate with lepton number minus baryon number, followed by lepton number, followed by baryon number. (Simple bosons and long-range forces correlate with zero lepton number and with zero baryon number.) Equation (98) shows an example of a $3 \mathrm{f0b} \rightarrow 1 \mathrm{f} 1 \mathrm{~b}$ interaction. Here, each of the three simple particles that correlates with $3 \mathrm{f}$ differs from the other two simple particles. One might assume that the $2 \mathrm{~T}^{+1 / 3}$ and $2 \mathrm{~T}^{-1 / 3}$ bosons annihilate each other.

$$
1 \mathrm{Q}_{+1 / 3 ; 0,-1 / 3}^{+1 / 3} \rightarrow 1 \mathrm{R}_{+1 / 3 ; 0,-1 / 3}^{0}+2 \mathrm{~T}^{+1 / 3}
$$




$$
1 \mathrm{C}_{-1 ;-1,0}^{+1}+1 \mathrm{R}_{+1 / 3 ; 0,-1 / 3}^{0}+1 \mathrm{Q}_{+1 / 3 ; 0,-1 / 3}^{-2 / 3} \rightarrow 1 \mathrm{Q}_{-1 / 3 ; 0,+1 / 3}^{+2 / 3}+2 \mathrm{~T}^{-1 / 3}
$$

In one scenario, $3 \mathrm{f} 0 \mathrm{~b} \rightarrow 1 \mathrm{f} 1 \mathrm{~b}$ interactions lead to effects similar to those that result from, in effect, combining equations (97) and (98). This scenario does not depend on the existence of $2 \mathrm{~T}$ (or, tweak) simple bosons. This scenario does not depend on the existence of $1 \mathrm{R}$ (or, arc) simple fermions. Equation (99) pertains.

$$
1 \mathrm{C}_{-1 ;-1,0}^{+1}+1 \mathrm{Q}_{+1 / 3 ; 0,-1 / 3}^{+1 / 3}+1 \mathrm{Q}_{+1 / 3 ; 0,-1 / 3}^{-2 / 3} \rightarrow 1 \mathrm{Q}_{-1 / 3 ; 0,+1 / 3}^{+2 / 3}+0 \text { I }
$$

In one scenario, $3 \mathrm{f} 0 \mathrm{~b} \rightarrow 1 \mathrm{f} 1 \mathrm{~b}$ interactions destroy antimatter quarks. This scenario does not depend on the existence of $2 \mathrm{~T}$ (or, tweak) simple bosons. This scenario does not depend on the existence of $1 \mathrm{R}$ (or, arc) simple fermions. Equation (100) shows an example of a $3 \mathrm{f} 0 \mathrm{~b} \rightarrow 1 \mathrm{f} 1 \mathrm{~b}$ interaction. Three antimatter fermions produce one matter fermion and a boson. Aspects of ongoing theory might suggest that the three quarks differ from each other by generation.

$$
31 \mathrm{Q}_{+1 / 3 ; 0,-1 / 3}^{-2 / 3} \rightarrow 1 \mathrm{C}_{+1 ;+1,0}^{-1}+2 \mathrm{~W}^{-1}
$$

We estimate a threshold energy for the scenario that equations (97) and (98) summarize. (Compare with estimating a threshold energy for the scenario that includes equation (80).) The threshold energy correlates with an energy to create two charged tweak particles. The threshold energy might be in the range of $416 \mathrm{GeV}$ to $442 \mathrm{GeV}$. (See equation (73).) A corresponding temperature is about $5 \times 10^{15}$ degrees Kelvin. Ongoing theory suggests that the creation of baryon asymmetry occurs at about $10^{-4}$ seconds after the big bang and that a temperature of about $10^{13}$ degrees Kelvin correlates with that time. (See reference [41].) Proposed theory might suggest a somewhat earlier time for the achievement of baryon asymmetry.

\subsection{Galaxies, components of galaxies, and ratios of dark matter to ordinary matter}

This unit suggests scenarios for the formation and evolution of galaxies. This unit discusses, for galaxies, observed ratios of dark matter effects to ordinary matter effects. This unit discusses some observations that might pertain regarding dark matter in the Milky Way galaxy.

We discuss galaxy formation and evolution scenarios and aspects pertaining to the amounts of ordinary matter and dark matter in galaxies. We assume that nature comports with at least one of PR6ISe modeling and PR36ISe modeling. (Neither ongoing theory nor PR1ISe modeling includes the notion of dark matter isomers. We think that it would be, at best, difficult to explain - based on for example $1 \mathrm{R} \otimes 2 \mathrm{U}$ dark matter - ratios, that observations suggest, of dark matter effects to ordinary matter effects.) For now, we de-emphasize some phenomena such as $1 \mathrm{R} \otimes 2 \mathrm{U}$ hadron-like particles and collisions between galaxies.

Models for galaxy formation and evolution might take into account the following factors - one-isomer repulsion (which correlates with the $4 \mathrm{G} 2468 \mathrm{a}$ and $4 \mathrm{G} 2468 \mathrm{~b}$ solutions), one-isomer attraction (which correlates with 4G246), two-isomer repulsion (which correlates with 4G48), six-isomer attraction (which correlates with 4G4), filaments (which correlate with effects of early universe baryon acoustic oscillations), statistical variations in densities of stuff, and collisions between galaxies. Modeling might feature a notion of a multicomponent fluid with varying concentrations of gas-like or dust-like components and of objects (such as stars, black holes, galaxies, and galaxy clusters) for which formation correlates significantly with six-isomer (or 4G4) attraction.

We focus on early-stage formation and evolution. For purposes of this discussion, we assume that we can de-emphasize collisions. We suggest the two-word term untouched galaxy for a galaxy that does not collide, before and during the time relevant to observations, with other galaxies. We emphasize formation scenarios and evolution scenarios for untouched galaxies.

We organize this discussion based on the isomer or isomers that originally clump based on, respectively, 4G246 attraction or 4G246 and 4G4 attraction. Each one of some galaxies correlates with an original clump that correlates with just one isomer. Multi-isomer original clumps are possible. Because of $4 \mathrm{G} 48$ repulsion, an upper limit on the number of isomers that an original clump features is three.

We discuss a scenario for the formation and evolution of a galaxy for which the original clump contains essentially just one isomer. Regarding this isomer, we use the word featured. We assume that PR6ISe modeling pertains. We assume that stuff that will become the galaxy is always in somewhat proximity with itself. We assume that no collisions between would-be galaxies or between galaxies occur. 
- Early on, each one of the six isomers of PR6ISe-span-one phenomena expands, essentially independently from the other isomers of PR6ISe-span-one phenomena, based on repulsion correlating with 4(1)G2468a and 4(1)G2468b.

- Then, each isomer of PR6ISe-span-one phenomena starts to clump, essentially independently from the other isomers of PR6ISe-span-one phenomena, based on attraction correlating with 4(1)G246.

- With respect to clumps correlating with any one isomer of PR6ISe-span-one phenomena, 4(2)G48 repels one other isomer of PR6ISe-span-one phenomena and repels some stuff correlating with itself.

- A galaxy forms based on a clump that contains mostly the featured isomer.

- The galaxy attracts and accrues, via 4(6)G4 attraction, stuff correlating with the four isomers of PR6ISe-span-one phenomena that the featured isomer does not repel. The galaxy can contain small amounts of stuff correlating with the isomer that the featured isomer repels.

We explore the extent to which the galaxy formation scenario comports with observations.

Observations of stars and galaxies tend to have bases in ordinary matter isomer $2 \gamma$ phenomena (or, readily observable electromagnetism). (The previous sentence de-emphasizes some observations - regarding collisions between black holes or neutron stars - that have bases in $4 \gamma$ phenomena.) People report ratios of amounts (or, densities) of dark matter to amounts (or, densities) of ordinary matter.

We discuss observations correlating with early in the era of galaxy formation.

Reference [19] reports zero-plus to one ratios. The observations have bases in the velocities of stars within galaxies and correlate with the three-word term galaxy rotation curves. Proposed theory suggests the above galaxy evolution scenario comports with this data. Presumably, other galaxies have one-isomer clumps that do not feature the ordinary matter isomer. Early on, those galaxies would not emit much $2 \mathrm{G}$ radiation that people could detect. People would not see such galaxies.

Reference [20] provides data about early stage galaxies. (See, for example, figure 7 in reference [20]. The figure provides two graphs. Key concepts include redshift, stellar mass, peak halo mass, and a stellar - peak halo mass ratio.) Data correlating with redshifts of at least seven suggests that some galaxies accrue, over time, dark matter, with the original fractions of dark matter being small. Use of reference [42] suggests that redshifts of at least seven pertain to times ending about 770 million years after the big bang. We suggest that our galaxy evolution scenario comports with this data.

We discuss observations correlating with later times.

Reference [21] discusses some MED09 spiral - or, disk - galaxies. A redshift of approximately $z=1.57$ pertains. (See reference [43].) The redshift correlates with a time of 4.12 billion years after the big bang. (We used reference [42] to calculate the time.) Reference [21] reports ratios of amount of dark matter to amount of ordinary matter of approximately four to one. The observations have bases in gravitational lensing.

To the extent that such an MED09 galaxy models as being nearly untouched, proposed theory offers the following possibility. The galaxy began based on a one isomer clump. The clump might have featured the ordinary matter isomer. The clump might have featured a dark matter isomer that does not repel ordinary matter. Over time, the galaxy accrued stuff correlating with the isomers that the original clump did not repel. Accrual led to a ratio of approximately four to one. Notions such as the following notions might pertain.

- Some ratios might not be as big as they might otherwise be because each one of the four relevant dark matter isomers of PR6ISe-span-one phenomena repels, via 4(2)G48, one relevant dark matter isomer of PR6ISe-span-one phenomena.

- Some ratios might not be as small as they might otherwise be because of contributions, which are independent of PR6ISe-span-one phenomena, of $1 \mathrm{R} \otimes 2 \mathrm{U}$ dark matter.

- Some ratios might reflect conditions specific to MED09 galaxies.

To the extent that such an MED09 galaxy models as not being untouched, proposed theory offers the following possibility. One type of collision merges colliding galaxies. One type of collision features galaxies that separate after exchanging material. For either type of collision, incoming galaxies having approximately four times as much dark matter as ordinary matter might produce outgoing galaxies having approximately four times as much dark matter as ordinary matter.

Reference [18] discusses the Dragonfly 44 galaxy. A redshift of $z=0.023$ pertains. The redshift correlates with a time of 13.45 billion years after the big bang. (We used reference [42] to calculate the 
Table 42: A method for cataloging not-significantly-collided galaxies that formed during the first few billion years after the big bang (with DM:OM denoting a ratio of amount of dark matter to amount of ordinary matter)

\begin{tabular}{lcccl}
\hline Original clump & $\begin{array}{c}\text { Eventual } \\
\text { DM:OM }\end{array}$ & $\begin{array}{c}\text { Relative } \\
\text { abundance }\end{array}$ & Spiral-like & Note \\
\hline 1IS: OM & $\sim 4$ & 1 & Many (?) & Visible early \\
1IS: DM1 & large & 1 & Many (?) & Dark matter galaxy \\
1IS: DMn & $\sim 4$ & 4 & Many (?) & Visible later \\
2IS including OM & $?$ & $x$ & Some (?) & Possibly visible early \\
2IS including DM1 & large & $x$ & Some (?) & Possibly, a dark matter galaxy \\
2IS: DMn, DMn' & $?$ & $x$ & Some (?) & Visible later \\
3IS including OM & $?$ & $y$ & Few (?) & Possibly visible early \\
3IS including DM1 & large & $y$ & Few (?) & Possibly, a dark matter galaxy \\
\hline
\end{tabular}

time.) People discuss the notion that ordinary matter accounts for perhaps as little as one part in 10 thousand of the matter in the galaxy. (See reference [44].) The observations have bases in light emitted by visible stars. This case correlates with the three-word term dark matter galaxy. Proposed theory suggests that this galaxy might have formed based on a core that included the isomer that repels the ordinary matter isomer.

Table 42 features a method for cataloging not-significantly-collided galaxies that formed during the first few billion years after the big bang. We use the one-element term not-significantly-collided to include possible collisions during the formation of original clumps and to exclude subsequent collisions. We use the one-element term spiral-like to include spiral dark matter galaxies. We use the two-element term possibly spiral-like to include the possibility that multi-isomer original clumps might produce other than spiral-like galaxies. (Each isomer might correlate with essentially just one axis of rotation but the axes might not align with each other. The three-element term other than spiral-like might correlate with the one-word term elliptical.) Some aspects of table 42 are conceptual or not necessarily completely rigorously expressed. The leftmost column describes the original clump. We do not specify mathematically boundaries between 1IS (or, one original isomer), 2IS (or, two original isomers), and 3IS (or, three original isomers). OM denotes the ordinary matter isomer. DM1 denotes the dark matter isomer that the ordinary matter isomer repels via the $4(2) \mathrm{G} 48$ long-range force. Each of DMn and DMn' can denote any one of the other four isomers that are relevant for the case of PR6ISe. Here, each of $n$ and $n^{\prime}$ is one of two, three, four, or five. Here, choices of $\mathrm{DMn}$ and $\mathrm{DMn}^{\prime}$ comport with the notion that DMn does not interact with $\mathrm{DMn}^{\prime}$ via $4(2) \mathrm{G} 48$. The next column estimates, based on assumptions such as a lack of collisions, ratios of dark matter density to ordinary matter density. (Collisions might tend to result in elliptical galaxies.) The estimates do not necessarily take into account phenomena related to $1 \mathrm{R} \otimes 2 \mathrm{U}$ dark matter. The relative abundances pertain billions of years ago. Each of $x$ and $y$ depends on natural phenomena and on the boundaries that one assumes between 1IS, 2IS, and 3IS. The column with the one-element label spiral-like has bases in some assumptions about the extent to which stuff correlating with a single isomer rotates around a single axis and about the extent to which, for multi-isomer original clumps, axes correlating with different isomers align with each other. Each one of the three words many, some, and few pertains regarding the galaxies that pertain for the relevant row in the table. Regarding the rightmost column, the following notions pertain. The word early might correlate with redshifts that exceed roughly seven (and, possibly, with some smaller redshifts). The word later might correlate with redshifts that do not exceed roughly seven (or, a number less than seven). We embrace an ongoing theory use of the three-word term dark matter galaxy.

The following notions pertain regarding other data of which we know. Here, the ratios are ratios of dark matter effects to ordinary matter effects.

- Reference [45] discusses six baryon-dominated ultra-diffuse galaxies that seem to lack dark matter, at least to the radius studied by gas kinematics via observations of light with a wavelength of 21 centimeters. These observations seem not to be incompatible with a scenario correlating with an original clump that features the ordinary matter isomer.

- Reference [46] discusses 19 dwarf galaxies that lack having much dark matter, from their centers to beyond radii for which ongoing theory suggests that dark matter should dominate. These observations measure r-band light that the galaxies emitted. These observations seem not to be 
incompatible with a scenario correlating with an original clump that features the ordinary matter isomer.

- The galaxy NGC1052-DF2 might correlate with a ratio of much less than one to one. (See reference [47].) The observation has bases in the velocities of stars - or, galaxy rotation curves. This observation seems not to be incompatible with the scenario correlating with an original clump that features ordinary matter. Reference [48] suggests, for NGC1052-DF2, that at least 75 percent of the stuff within the half mass radius is dark matter. To the extent this suggestion comports with nature, phenomena related to NGC1052-DF2 might correlate with results that reference [21] discusses regarding some MED09 galaxies. (See discussion above regarding MED09 galaxies.) Proposed theory seems to be not incompatible with either ratio. Proposed theory might not, based on known data, be able to refute either ratio.

- The galaxy NGC1052-DF4 might correlate with a ratio of much less than one to one. (See reference [49].) The observation has bases in the velocities of stars - or, galaxy rotation curves. This observation seems not to be incompatible with the scenario correlating with an original clump that features ordinary matter.

- The compact elliptical galaxy Markarian 1216 has an unexpectedly large amount of dark matter in its core and may have stopped accumulating each of ordinary matter and dark matter approximately 4 billion years after the big bang. (See references [22] and [50].) Observations feature the X-ray brightness and temperature of hot gas. This galaxy might correlate with the case correlating with the three-element term 3IS including OM and with an original clump that features three isomers. One isomer would be the ordinary matter isomer. Around the time that the galaxy stopped accruing material, there was - near the galaxy - essentially nothing left for the galaxy to attract via 4(6)G4.

- The galaxy XMM-2599 stopped producing visible stars by approximately 1.8 billion years after the big bang. (See reference [51].) People speculate regarding a so-called quenching mechanism. Proposed theory might suggest that phenomena similar to phenomena that might pertain regarding Markarian 1216 might pertain regarding XMM-2599.

- People report other data. We are uncertain as to the extent proposed theory provides insight that ongoing theory does not provide.

- One example pertains to early stages of galaxies that are not visible at visible light wavelengths. (See reference [52].) Observations feature sub-millimeter wavelength light. We might assume that proposed theory galaxy formation scenarios comport with such galaxies. We are not certain about the extent to which proposed theory provides insight regarding subtleties, such as regarding star formation rates, correlating with this example.

- We are uncertain as to the extent to which proposed theory provides insight regarding possible inconsistencies - regarding numbers of observed early stage galaxies and numbers of later stage galaxies - that correlate with various observations and theories. (For a discussion of some possible inconsistencies, see reference [53].)

- We are uncertain as to the extent to which proposed theory provides insight regarding the existence of two types - born and tidal - of ultra-diffuse galaxies. (See reference [54].)

Observations that we discuss above indicate that some galaxies do not exhibit dark matter halos. Theory that we discuss above comports with the notion that some galaxies do not exhibit dark matter halos.

Discussion above is not incompatible with the notion that visible stars do not include much dark matter.

Discussion above is not incompatible with the notion that some black holes that form based on the collapse of stars might originally correlate with single isomers. Discussion above is not incompatible with the notion that supermassive black holes might contain material correlating with more than one isomer. (Perhaps, note references [55] and [56].)

We suggest that proposed theory might provide insight about other aspects regarding black holes. People suggest gaps in understanding about the formation of intermediate-mass and large-mass black holes. (Perhaps note reference [57].) Proposed theory suggests the possibility that the 4G(1)246 attractive component of long-range forces plays key roles in the early formation of some intermediate-mass and large-mass black holes. 
Regarding the coalescing of two black holes, proposed theory suggests that people might be able to estimate the extent to which $4 \mathrm{G} 48$ repulsion pertains. Effects of $4 \mathrm{G} 48$ repulsion would vary based on the amounts of various isomers that each of a pair of colliding black holes features.

We discuss other effects, within galaxies, that might correlate with dark matter.

People study globular cluster systems within ultra diffuse galaxies. Regarding 85 globular cluster systems in ultra diffuse galaxies in the Coma cluster of galaxies, reference [58] suggests that 65 percent of the ultra diffuse galaxies are more massive than people might expect based on ongoing theory relationships, for so-called normal galaxies, between stellar mass and halo mass. We are uncertain as to the extent to which proposed theory might explain this result. For example, proposed theory might suggest that phenomena related to isomers might play a role. (See, for example, table 42.) Higher-mass clusters might tend to feature more dark matter isomers (or tend to feature more material that correlates with such isomers) than do lower-mass clusters.

People look for possible local effects, within the Milky Way galaxy, that might correlate with dark matter.

For one example, data regarding the stellar stream GD-1 suggests effects of an object of $10^{6}$ to $10^{8}$ solar masses. (See reference [23].) Researchers tried to identify and did not identify an ordinary matter object that might have caused the effects. The object might be a clump of dark matter. (See reference [59].) Proposed theory offers the possibility that the object is an originally dark matter centric clump of stuff (that might include at least one dark matter black hole).

For other examples, people report inhomogeneities regarding Milky Way dark matter. (See references [59] and [60].) Researchers note that simulations suggest that such dark matter may have velocities similar to velocities of nearby ordinary matter stars. Proposed theory suggests that these notions are not incompatible with proposed theory notions that dark matter stars, which would be similar to ordinary matter stars, exist.

\subsection{Galaxy clusters, ratios of dark matter effects to ordinary matter effects, and filaments}

This unit discusses, for galaxy clusters, observed ratios of dark matter effects to ordinary matter effects. This unit notes possible implications, regarding filaments, of dark matter baryon acoustic oscillations.

Regarding some galaxy clusters, people report inferred ratios of dark matter effects to ordinary matter effects.

References [61] and [62] report ratios of five-plus to one. The observations have bases in gravitational lensing.

Reference [63] reports, for so-called massive galaxy clusters, a ratio of roughly 5.7 to one. (Perhaps note reference [64].) The observations have bases in X-ray emissions.

We suggest that proposed theory is not necessarily incompatible with these galaxy cluster centric ratios.

Reference [65] suggests a formula that correlates - across 64 galaxy clusters - dark matter mass, hot gas baryonic mass (or, essentially, ordinary matter mass), and two radii from the centers of each galaxy cluster. The reference suggests that the formula supports the notion of a correlation between dark matter and baryons. Proposed theory might suggest a correlation, based on proposed similarities between most dark matter and ordinary matter. We are uncertain as to the extent to which people might consider that the formula supports this aspect of proposed theory.

Proposed theory is not necessarily incompatible with the ongoing theory notion that ordinary matter centric baryon acoustic oscillations contributed to the formation of filaments.

Regarding models for which $\mathrm{n}$ (as in PRnISe) exceeds one, each of the five dark matter isomers of PR6ISe-span-one phenomena has its own baryon-like particles and its own PR1ISe-like photon physics. Proposed theory suggests, for models for which $\mathrm{n}$ (as in PRnISe) exceeds one, that dark matter baryonlike acoustic oscillations occurred in the early universe. Proposed theory suggests that dark matter baryon-like acoustic oscillations contributed (along with ordinary matter baryon acoustic oscillations) to the formation of filaments.

\subsection{Dark energy density}

This unit discusses the notion that dark energy densities might correlate with aspects related to aye (or, 0I) bosons, with dark matter, or with dark energy stuff. 
Equation (101) shows an inferred ratio of present density of the universe of dark energy to present density of the universe of dark matter plus ordinary matter plus (ordinary matter) photons. (Reference [17] provides the four items of data.) From a standpoint of each of ongoing theory and proposed theory, equation (101) does not include neutrino density of the universe. From a standpoint of proposed theory, $\Omega_{\mathrm{c}}$ includes effects correlating with $1 \mathrm{R} \otimes 2 \mathrm{U}$ hadron-like particles. (See equation (87).) From a standpoint of proposed theory, for models for which $\mathrm{n}$ (as in PRnISe) exceeds one, $\Omega_{\mathrm{c}}$ includes PR1ISe-like photons centric to dark matter. We know of no inferences that would not comport with a steady increase, regarding the inferred ratio correlating with equation (101), from approximately zero, with time since somewhat after the big bang. (Reference [36] implies a ratio of approximately zero correlating with 380 thousand years after the big bang.)

$$
\Omega_{\Lambda} /\left(\Omega_{\mathrm{c}}+\Omega_{\mathrm{b}}+\Omega_{\gamma}\right) \approx 2.3
$$

Some aspects of ongoing theory try to correlate inferred dark energy densities of the universe with phenomena correlating with terms such as vacuum energy, vacuum fluctuations, or quintessence. Proposed theory does not necessarily embrace notions such as vacuum energy. (Double-entry modeling may obviate needs to consider notions such as vacuum energy.)

Aspects related to aye (or, 0I) bosons might lead to phenomena similar to effects that ongoing theory might correlate with vacuum energy, vacuum fluctuations, or quintessence. (See discussion related to equation (53).) Ongoing theory correlates some of those effects with data about dark energy densities. To the extent that phenomena correlating with aye bosons suffice to explain dark energy densities, there might not necessarily be a need to consider PR36ISe modeling. Assuming that such phenomena might not adequately explain non-zero dark energy density, we discuss possibilities for other proposed theory aspects that might explain non-zero dark energy density.

For PR6ISe modeling, proposed theory includes the notion of 2(6)G248, whereas ongoing theory correlates with the notion of 2(1)G248. We suggest that the difference, in proposed theory, between 2(6)G248 and 2(1)G248 might correlate with nature's producing effects, regarding CMB, that people correlate, via ongoing theory, with some non-zero dark energy density. The difference correlates with interactions between ordinary matter and dark matter.

For PR36ISe modeling, differences between 2(>1)G $\Gamma$ and 2(1)G correlate with interactions between ordinary matter plus dark matter and doubly dark matter. For example, half or somewhat less than half of the effect that reference [24] reports correlates with 2G68 interactions correlating with one doubly dark matter isomer of hydrogen atoms. Also, any span-36 phenomena would correlate with interactions between ordinary matter plus dark matter and doubly dark matter. Neutrinos and $1 \mathrm{R} \otimes 2 \mathrm{U}$ particles might provide for such interactions. (See table 40.) In effect, dark energy density correlates with a notion of dark energy stuff. Modeling suggests an upper bound of approximately five regarding, in effect, a possible future value for the ratio that correlates with equation (101).

Proposed theory comports with the notion that ratios of inferred density of dark energy to inferred density of ordinary matter plus dark matter grow with respect to the time, since the big bang, correlating with observed phenomena upon which people base the inferences. Data that reference [36] shows supports the notion of such growth. Inferences that reference [66] discusses might comport with this aspect of proposed theory.

\subsection{High-mass neutron stars}

This unit suggests proposed theory that might explain some aspects regarding high-mass neutron stars.

An approximate minimal mass for a neutron star might be $1.1 M_{\odot}$. (See reference [67].) The symbol $M_{\odot}$ denotes the mass of the sun. An approximate maximum mass for a neutron star might be $2.2 M_{\odot}$. (See reference [68].

Observations correlate with most known neutron star pairs having masses in the range that equation (102) shows and one neutron star pair having a mass of about 3.4 solar masses. (See references [69] and [70].) Here, $M$ denotes the mass of a pair. The 3.4 number results from the second detection via gravitational waves of a merger of two neutron stars. People assign the name GW190425 to that detection.

$$
2.5 M_{\odot} \lesssim M \lesssim 2.9 M_{\odot}
$$

People speculate, based on the GW190425 result, about needs for new theory regarding neutron stars. (See reference [69].) 
To the extent that people need new theory regarding high-mass neutron stars, proposed theory might suggest useful new theory. Some high-mass neutron stars might, in effect, result from mergers of neutron stars, with each merging neutron star correlating with an isomer of charge (or, of charged simple particles) that differs from the isomer pertaining to each other neutron star that forms part of the merger. The notion that the span of $4 \mathrm{G} 4$ exceeds one (and is six) underlies this possibility. The notion that the PR6ISe span of gluons (or, $2 \mathrm{U}$ simple particles) is six might underlie this possibility. The span of quarks (or, 1Q simple particles) is one.

\subsection{Phenomena before inflation}

This unit discusses phenomena that might correlate with times before the inflationary epoch.

We speculate about phenomena that might have preceded the inflationary epoch.

Proposed theory correlates an $S U(5)$ symmetry with conservation of energy. The number of generators of $S U(5)$ is 24. Equation (103) might pertain. Here, $g_{U(1)}$ denotes the number of generators for $U(1)$ and equals two. The number 24 equals six times two times two. One factor of two might correlate with the possibility for two values for handedness for baryons. One factor of two might correlate with the possibility for two values of handedness for leptons. The factor of six might correlate with the relevance of six isomers regarding color charge. (See discussion regarding equation (142) and discussion regarding table 49.)

$$
\left(g_{7} / g_{3}\right) \times g_{U(1)} \times g_{U(1)}
$$

To the extent that such aspects correlating with the $S U(5)$ symmetry comport with nature, one might consider models that suggest 24 somewhat similar entities. People might apply the two-word term our universe to one of the 24 somewhat similar entities.

We note, but do not pursue further, the possibility that theory might correlate, with the big bang, a transition from $\sigma_{-}=-1$ modeling to $\sigma_{-}=+1$ modeling, for a $\sigma_{-}$that has roles somewhat similar to $\sigma$. This transition might involve, in effect, a decoupling of the possible 24 isomers.

\subsection{Directly detecting dark matter and doubly dark matter}

This unit discusses aspects of extant approaches for directly detecting dark matter. This unit discusses possible new approaches for directly detecting dark matter or doubly dark matter.

Discussion related to equation (80) pertains to the possibility of making dark matter.

We discuss possibilities for observing dark matter effects without creating dark matter.

We are aware of various efforts to directly detect dark matter. Some efforts look for WIMPs. We are uncertain as to the extent to which these efforts might be able to detect $1 \mathrm{R} \otimes 2 \mathrm{U}$ hadron-like particles. Some efforts look for axions. We are uncertain as to the extent to which these efforts might attribute axion sightings to effects that correlate with the difference that equation (104) shows.

$$
2(6) \mathrm{G} 248 \neq 2(1) \mathrm{G} 248
$$

Proposed theory suggests new possibilities for directly detecting dark matter or doubly dark matter. To the extent that PR6ISe pertains to nature and PR36ISe does not pertain to nature, the following discussion pertains to detecting dark matter. To the extent that PR36ISe pertains to nature, the following discussion pertains to detecting doubly dark matter. The basis for one possibility is the difference between 2(6)G248 and 2(1)G248. Here, a detector might feature a rotating (or, precessing) magnetic dipole moment, with the axis of rotation perhaps being orthogonal (and not being parallel) to the axis correlating with the magnetic dipole. Independent of that possible means for detection, people might try to infer 2(6)G248 phenomena correlating with precessing dark matter magnetic fields (or - for the PR36ISe case - 2(6)G248 phenomena correlating with precessing doubly dark matter magnetic fields). A basis for another possibility is the difference between 2(2)G68 and 2(1)G68. Proposed theory suggests that 2G68 correlates with, at least, some atomic transitions.

\section{Relationships between various theories and models for motion}

This unit discusses aspects of kinematics modeling and aspects of dynamics modeling. This unit shows branch points between proposed theory modeling for motion and ongoing theory modeling for motion. This unit discusses dynamics modeling for hadron-like particles, nuclear physics, and quantum transitions. 
This unit suggests limits on the applicability of general relativity. This unit discusses possible modeling regarding fissionable or bound-state multicomponent objects. This unit suggests modeling regarding refraction. This unit suggests details regarding modeling pertaining to the strong interaction within hadron-like particles.

\subsection{Kinematics modeling and dynamics modeling}

This unit discusses aspects of kinematics modeling and aspects of dynamics modeling.

Kinematics models and dynamics models tend to discuss translational motion.

Kinematics models feature free-ranging objects in free environments. Dynamics models feature objects in confined environments.

We review kinematics and dynamics modeling that we discuss above in this essay. Modeling features simple particles and long-range forces. We tend to avoid dynamics modeling. We tend to feature models for which kinematics conservation laws pertain to individual objects. We tend to avoid choosing kinematics models.

We anticipate correlating proposed theory with ongoing theory kinematics and dynamics.

Kinematics models can correlate with classical physics or with quantum physics. Kinematics models can correlate with Newtonian physics modified to limit the speed, of the free-environment transmission of effects, to the speed of light. Kinematics models can correlate with special relativity. Kinematics models can correlate with general relativity. Kinematics models can be linear in energy or quadratic in energy. The Dirac equation is linear in energy. The Klein-Gordon equation is quadratic in energy.

The following points pertain.

- Proposed theory might be compatible with all choices of kinematics models.

- Special relativity features boost symmetry. In the context of ongoing theory or in the context of proposed theory, boost symmetry correlates with an additional SA-side $S U(2)$ symmetry. The double-entry bookkeeping aspect of proposed theory can accommodate boost symmetry by adding one SA-side pair of oscillators that correlates with any one of no symmetry, $U(1)$ symmetry, or $S U(2)$ symmetry. We use the two-element phrase boost-related symmetry to correlate with those three possibilities. The correspondingly added TA-side pair of harmonic oscillators can correlate with no symmetry. Each of the TA-side addition and the SA-side addition might correlate with modeling and might not correlate with observable phenomena.

We discuss some aspects regarding modeling for the kinematics and dynamics of multicomponent systems.

We distinguish from each other kinematics and dynamics. For an object, kinematics correlates with externally observed motion. Kinematics symmetries include conservation of energy, conservation of angular momentum, and conservation of momentum. Dynamics correlates with internal properties. For the object, we posit that dynamics symmetries include conservation of energy, conservation of angular momentum, and conservation of momentum. We posit that proposed theory can treat two sets, each of at least three conserved aspects, as being distinct.

We consider cases of multicomponent objects that involve at least two peer component objects. Here, there are four possibly relevant levels of symmetries - kinematics for the multicomponent object, dynamics for the multicomponent object, kinematics for each of the component objects, and dynamics for each of the component objects that is not an elementary particle. In keeping with the notion of object, each of kinematics for the multicomponent object, dynamics for the multicomponent object, and dynamics for each component object (that is not an elementary particle) correlates with each of the three conserved aspects. However, kinematics for each of the component objects does not necessarily correlate with a complete set of the three conserved aspects.

For example, we consider a multicomponent object that is a binary star system. Kinematics for each star does not correlate with conservation of momentum.

In general, compared with dynamics symmetries for the multicomponent object, the two stars collectively contribute one too many instance of each of conservation of energy symmetry, conservation of angular momentum symmetry, and conservation of momentum symmetry. Modeling can re-assign the extra three symmetries to a field - in this case a gravitational field - that correlates with interactions between the two stars.

We consider the case of a pion. We consider the pion to be a multicomponent object for which each of the two components is a quark. (Here, we do not distinguish between matter quarks and antimatter quarks.) We consider two branches for this case. For each branch, the field correlates with bosons that correlate with the strong interaction and the electromagnetic interaction (or, with gluons and photons). 
For one branch, kinematics for each of quarks and bosons correlates with the three conserved aspects. We suggest that ongoing theory QCD (or, quantum chromodynamics) and (to some extent) ongoing theory QED (or, quantum electrodynamics) correlate with this branch. (It might be prudent to recall that ongoing theory tends to feature modeling that is linear in energy, momentum, and mass, whereas proposed theory tends to be quadratic in energy, momentum, and mass.) Ongoing theory QCD modeling and QED modeling involve virtual particles. This branch might correlate with an attempt to enforce a notion of $\sigma=+1$ for each of kinematics for quarks and kinematics for gluons.

For the other branch, proposed theory suggests correlating one TA-side $S U(3)$ symmetry and one SAside $S U(2)$ symmetry with kinematics for quarks and correlating one TA-side $S U(3)$ symmetry and one SA-side $S U(2)$ symmetry with kinematics for bosons. (The notion of $S U(3)$ correlates with a notion of two additional TA-side oscillators and with thinking that correlates with the $S U(5)$ symmetry that table 14 lists.) Those symmetries combine to correlate with the appropriate three symmetries correlating with the three conserved aspects for dynamics of the pion. This branch correlates with the proposed theory notion that each of quarks and gluons correlates with $\sigma=-1$. (See table 1 and discussion related to table 44.) This branch correlates with the notion that some aspects of proposed theory do not necessarily need to consider notions of virtual particles. This branch seems to correlate with modeling that does not necessarily correlate with the three kinematics conservation laws pertaining regarding dynamics within quarks or regarding dynamics within gluons. However, proposed theory does not necessarily need to consider notions of dynamics within simple particles.

\subsection{Perspective regarding quantum modeling and kinematics modeling}

This unit discusses relationships between quantum modeling and kinematics modeling.

Proposed theory might seem to include a problem regarding possible dissonance between modeling pertaining to quantum interactions and modeling pertaining to kinematics.

For example, proposed theory suggests that $4 \gamma$ interactions do not necessarily directly lead to neutrino oscillations. Yet, kinematics modeling needs to allow for gravity to alter the trajectories of neutrinos. Symbolically, equations (105) and (106) might pertain.

$$
1 \mathrm{~N}+4 \gamma \nrightarrow 1 \mathrm{~N}+4 \gamma \text { pertains regarding quantum mechanical transitions }
$$

$$
1 \mathrm{~N}+4 \gamma \rightarrow 1 \mathrm{~N}+4 \gamma \text { pertains regarding translational motion }
$$

Similar concerns might pertain regarding photons. Similar concerns might pertain regarding the aye (or, 0I) boson. For the aye boson, proposed theory suggests that interactions with 4G2468a and 4G2468b lead to effects during and just after inflation. (See discussion related to equation (95).) However, aye bosons have no mass (and presumably have no mass-centric moment of inertia) and might not interact quantum mechanically with $4 \gamma$.

We think that resolution lies in the notion that motion of a simple particle or of a photon can occur without the simple particle or photon undergoing a quantum transition. Proposed theory modeling regarding quantum transitions and ongoing theory modeling regarding motion have some independence from each other. Discussion regarding equations (17) and (18) provides an example regarding phenomena related to $\Sigma \mathrm{G}$ (or, the G-family).

\subsection{Possible complements to ongoing theory QFT, QED, and QCD}

This unit summarizes aspects of possible proposed theory complements to ongoing theory QFT (or, quantum field theory), QED (or, quantum electrodynamics), and QCD (or, quantum chromodynamics).

We assume a definition of QFT that does not necessarily imply a correlation with special relativity. (See, for example, reference [71].)

Generally, the following notions pertain.

- Proposed QFT suggests simple particles, long-range forces, and interaction vertices that nature embraces or might embrace.

- Ongoing QFT uses descriptions of elementary particles and of interaction vertices to suggest models for motions of simple particles, motions of long-range force bosons, and dynamics within atoms and hadrons. 
- Proposed QFT suggests means to summarize some aspects of interactions in terms of interaction vertices that people might consider to be aggregates of ongoing theory interaction vertices.

- Proposed QFT suggests means to summarize some aspects of interactions in terms of potentials that people might consider to be aggregates of ongoing theory interaction vertices.

The following statements summarize aspects of possible complements to ongoing theory QFT.

- Proposed QFT interaction vertices can correlate with aspects of PDE modeling.

- Proposed QFT interaction vertices do not necessarily correlate only, with respect to coordinates, with points. Vertices can correlate with objects that model as existing within a region having non-zero temporal extent and non-zero spatial extent.

- Proposed QFT does not necessarily need to consider notions of virtual particles.

- PDE modeling correlates with aspects of the four ongoing theory fundamental forces.

- Proposed QFT correlates with the following notions.

- Modeling correlating with the notion of objects in free environments needs to embrace, for each of those objects, all three ongoing theory kinematics conservation laws.

- Modeling correlating with the notion of objects in confined environments does not necessarily need to embrace, for each of those objects, all three ongoing theory kinematics conservation laws. Modeling correlating with the notion of objects in confined environments does not necessarily need to embrace the notion of interaction vertices.

The following statements summarize aspects of possible complements to ongoing theory QED.

- Proposed QED can describe anomalous magnetic dipole moments (and other aspects of physics) via sums over finite numbers of terms. (See discussion related to equation (66).)

- Proposed QED might point to new approaches to atomic physics.

- The next two sentences provide bases for an approach. The $\Omega_{S A} r^{-2}$ term in equation (27) might correlate, at least somewhat, with the square of the potential that impacts an electron. In equation (26), the limit that equation (107) shows can pertain while $\left(\xi_{S A}^{\prime} / 2\right)\left(\eta_{S A}\right)^{2}$ remains a non-zero constant. (The strong force is not relevant to the relevant aspects of atomic physics. The limit correlates with, in effect, de-emphasizing non-residual strong force aspects of the strong interaction. Presumably, $\left(\xi_{S A}^{\prime} / 2\right)\left(\eta_{S A}\right)^{2}$ is proportional to each of $\hbar^{2}$ and energy squared.) An approach might lead to insight regarding allowed states. An approach might not correlate well with abilities to compute energies for states.

$$
\left(\xi_{S A}^{\prime} / 2\right)\left(\eta_{S A}\right)^{-2} \rightarrow 0^{+}
$$

The following statement summarizes aspects of possible complements to ongoing theory nuclear physics.

- Proposed QFT might describe allowed states for atomic nuclei, based on PDE modeling. (See discussion related to equation (108).)

The following statement summarizes aspects of possible complements to ongoing theory QCD.

- Proposed QCD might describe allowed states for hadron-like particles, based on PDE modeling. (See discussion related to table 14 and discussion related to table 44.)

\subsection{Dynamics models for hadron-like particles}

This unit discusses an approach, compatible with proposed theory, for modeling the kinematics, in hadrons, of quarks and gluons. This unit also calls attention to possible differences between modeling for the dynamics of hadron-like particles that contain no more than three quarks and modeling for the dynamics of hadron-like particles that contain more than three quarks.

We discuss the notion that each hadron-like particle that includes no more than three quarks (or, 1Q particles) and arcs (or, 1R particles) does not include both quarks and arcs. Discussion related to table 23 suggests that a hadron-like particle has a charge for which the magnitude is either zero or a non-zero 
integer multiple of $\left|q_{e}\right|$ and a baryon number that is either zero or a non-zero integer multiple of one. For a hadron-like particle that includes no more than three quarks and arcs, the restrictions to integer charge and integer baryon number preclude the presence of both quarks and arcs. A tetraquark might contain a matter-and-antimatter pair of quarks and a matter-and-antimatter pair of arcs.

We discuss modeling for dynamics in hadrons that contain no more than three quarks.

Ongoing theory QCD modeling correlates with symmetries, for each of quarks and gluons, that correlate with special relativity. Proposed theory suggests possibilities for modeling that correlates one subset of those symmetries with kinematics for quarks and another subset of those symmetries with kinematics for gluons. (See discussion related to table 14 and discussion related to table 44.) This proposed theory modeling correlates with the notion that neither one of quarks and gluons behaves like a simple particle for which $\sigma=+1$.

Reference [72] suggests opportunities to improve understanding regarding modeling that might explain the masses of hadrons such as protons. Proposed theory suggests concepts that might help regarding such opportunities. One concept correlates with avoiding relying on modeling that correlates with special relativity. (See discussion nearby above.) One concept correlates with equations (26) and (27) and with $D=3$. Here, the term that is proportional to $r^{2}$ might correlate with the square of a potential. For a two-quark hadron, the potential associated with one quark affects the other quark. For a three-quark hadron, the potential associated with two quarks affects the third quark.

We discuss modeling for dynamics in hadrons that contain more than three quarks.

Reference [73] suggests that some of the dynamics within at least some pentaquarks correlates with the dynamics for a system composed of a meson-like particle and a baryon-like particle. The meson-like particle features a matter quark and an antimatter quark. The baryon-like particle features three matter quarks. Aspects that proposed theory correlates with the pie simple particle and with the cake simple particle might play roles in such dynamics.

Modeling might consider that, if hexaquarks exist, some hexaquarks have parallels to atomic nuclei.

\subsection{Dynamics models for nuclear physics}

This unit suggests possibilities for developing proposed theory models for atomic nuclei.

We discuss nuclear physics.

Ongoing theory bases some aspects of modeling on notions of a Pauli exclusion force and on notions of a Yukawa potential. Ongoing theory correlates these effects with notions of a residual strong force. The Pauli exclusion force keeps hadrons apart from each other. The Yukawa potential attracts hadrons to each other. Modeling suggests virtual pions as a source for the Yukawa potential.

Proposed theory does not necessarily correlate with a Pauli exclusion force or with notions of virtual pions. Cake (or, $0 \mathrm{~K}$ ) bosons might correlate with repulsion between hadrons. From a standpoint of modeling, $0 \mathrm{~K}$ bosons might correlate with interactions with colorless color charge or white color charge. From a standpoint of modeling, $0 \mathrm{~K}$ bosons might correlate with the identity operator that the relevant (ongoing theory and proposed theory) gluon-related $S U(3)$ symmetry lacks. From a standpoint of modeling, pie (or, 0P) bosons might correlate with attraction between hadrons. The attraction might correlate with a PDE-centric expression proportional to the term that equation (108) shows and with a Yukawa-like $\exp \left(-r /\left|\eta_{S A}\right|\right)$ potential. (See discussions related to equations (38) and (44).) The Yukawa-like potential can pertain for times longer than it would take light to traverse an atomic nucleus. We suggest that $v_{c}<c$ pertains. (See equation (40).) From a standpoint of modeling, 0P bosons might correlate with the identity operator that the $S U(2)$ component of a relevant weak interaction $S U(2) \times U(1)$ symmetry lacks.

$$
\exp \left(-\operatorname{tr} /\left(\left|\eta_{T A}\right| \cdot\left|\eta_{S A}\right|\right)\right)
$$

People might develop models, for atomic nuclei, based on potentials that correlate with aspects that techniques paralleling ongoing theory techniques might attribute to cake virtual particles and pie virtual particles. We are uncertain as to aspects regarding the potential that would correlate with cake bosons. We are uncertain as to the extent to which models would improve on ongoing techniques.

\subsection{Dynamics models for quantum transitions}

This unit discusses the possibility that aspects of proposed theory pertain to temporal aspects of quantum transitions. 
People discuss the extent to which quantum transitions correlate with non-zero time intervals. (See, for example, reference [74].) People may have observed quantum transitions that take non-zero time. (See reference [75].)

Proposed theory suggests that people can model such aspects of transitions via volume-like vertices. Modeling that features volume-like vertices might parallel temporal aspects of equation (108). (See discussion regarding equation (38).)

\subsection{Models for interactions with gravity}

This unit discusses models for non-quantum interactions between objects and gravity.

Equation (109) shows Newtonian modeling regarding gravity. Each $m$ denotes the mass of an object. The symbol $\vec{r}$ denotes a vector pointing from object one to object two. The symbol $r$ denotes the distance between the two objects and the length of the vector. The symbol $\vec{F}$ denotes the force that object one exerts on object two. The symbol $\vec{a}$ denotes the acceleration that pertains regarding the motion of object two.

$$
\left(G_{N} m_{1} m_{2} / r^{2}\right)(-\vec{r} / r)=\vec{F}=m_{2} \vec{a}
$$

The factor $m_{2}$ appears in each of the left-most and right-most parts of equation (109). Observations show that gravity bends the paths of light. Equation (110) pertains, including for photons. Equations (111) and (112) might pertain and might point toward aspects of general relativity. The symbol $E$ denotes energy. The symbol $\vec{P}$ denotes momentum.

$$
\begin{gathered}
\left(G_{N} m_{1} / r^{2}\right)(-\vec{r} / r)=\vec{a} \\
-\left(G_{N} E_{1} E_{2} / r^{2}\right) \overrightarrow{\hat{r}}=\vec{F}=E_{2} \vec{a} \\
\left(E_{-}\right)^{2}=\left(m_{-} c^{2}\right)^{2}+\left(\overrightarrow{P_{-}} \cdot \overrightarrow{P_{-}}\right) c^{2}
\end{gathered}
$$

We consider the motion of a simple boson for which $\sigma=+1$ or of a quanta that correlates with a longrange force. ALG modeling pertains. Quantum excitation or de-excitation need not necessarily pertain regarding effects of gravity. (See discussion regarding equations (17) and (18).) Modeling regarding energy and momentum might pertain. (See, for example table 14. Discussion related to equation (114) notes notions that might underlie such modeling.)

\subsection{General relativity and large-scale physics}

This unit suggests limits regarding the applicability of modeling based on general relativity. This unit suggests possible opportunities for research regarding modeling various aspects of large-scale physics.

While general relativity comports with various phenomena, people discuss possible problems regarding the applicability of general relativity to large-scale physics. (See, for example, reference [26].) Also, people express other concerns regarding modeling pertaining to large-scale physics. For example, reference [8] alludes to possible concerns correlating with the Hubble constant (or, a Hubble parameter).

Proposed theory offers possible insight and resolution regarding such concerns.

Table 43 lists aspects related to $4 \mathrm{G} \Gamma$ solutions. In the context of PR1ISe modeling, each row in the table points to a possible correlation with general relativity. For each row, the extent to which the possible correlation pertains might be an open question. People associate the two-element term Lense-Thirring effect with the two-element term rotational frame-dragging. The Einstein field equations allow solutions that correlate with repulsion. (See reference [13].)

We consider modeling that might pertain to large-scale phenomena for other than the very early universe.

We assume that general relativity pertains regarding PR1ISe modeling, including $4 \gamma$ aspects of PR1ISe modeling.

We consider the case of PR6ISe modeling.

Galaxy clusters tend to have equal amounts of stuff correlating with each of the six isomers.

We consider modeling related to the current multibillion-year era of accelerating rate of expansion of the universe. (See table 41.) We consider two similar objects that have equal amounts of stuff correlating with each of the six isomers and that are at least as large as galaxy clusters. We assume that proposed 
Table 43: Possible correlations, regarding PR1ISe modeling, with general relativity

\begin{tabular}{cc}
\hline Aspect regarding proposed theory & Aspect regarding general relativity \\
\hline $4 \mathrm{G} 48$ & rotational frame-dragging \\
$4 \mathrm{G} 48$ repulsion & Einstein field equations \\
$4 \mathrm{G} 246$ attraction & Einstein field equations \\
$4 \mathrm{G} 2468 \mathrm{a}$ and $4 \mathrm{G} 2468 \mathrm{~b}$ repulsion & Einstein field equations \\
$4 \mathrm{G}$ components other than $4 \gamma$ components & Einstein field equations \\
\hline
\end{tabular}

theory pertains. We assume that ongoing theory general relativity pertains. If the objects are adequately far apart, the dominant $4 \gamma$ force component is $4 \mathrm{G} 4$. The $4 \mathrm{G} 4$ component has a span of six. By assumption, modeling based on general relativity suffices. If the objects are adequately close to each other, $4 \mathrm{G} 48$ can be the dominant $4 \gamma$ force. The $4 \mathrm{G} 48$ component has a span of two. The ratio of two to six is onethird. Each span-one simple particle within one object experiences a force that is one-third the force that might correlate with modeling based on general relativity. General relativity might over-estimate the interaction between the two objects, unless one adjusts something like the cosmological constant or a relationship between density and pressure. Absent such an adjustment, modeling would overestimate rates of expansion. (We use the word rates - and not the word rate - because the rate changes with time.)

Regarding a possible model that includes both the multibillion-year era of decelerating rate of expansion and the current multibillion-year era of accelerating expansion, similar problems might arise. The 4 G246 attractive component of $4 \gamma$ has a span of one isomer. Tuning a model to the era of deceleration might produce a model that underestimates effects that lead to the (accelerating) rates that correlate with the current era.

We consider the case of PR36ISe modeling. Six isomers of 4(6)G4 pertain. General relativity might pertain somewhat for each of the six PR6ISe-like isomers. General relativity would not pertain across PR6ISe-like isomers.

Concepts such as those we just mentioned might point to opportunities for observational and theoretical research regarding each of the following topics and regarding relationships between the following topics - the domain of applicability of general relativity; equations relating pressures to densities; the notion and applicability of the concept of a Hubble parameter; notions regarding geodesic motion; and the spans and the strengths of forces correlating with the 4G48, 4G246, 4G2468a, and 4G2468b solutions.

We de-emphasize in this essay possible problems with trying to, in effect, extend modeling, based on general relativity, to very early times after the big bang.

\subsection{Aspects of dynamics modeling regarding hadron-like particles}

This unit discusses modeling regarding components of hadron-like particles. This unit illustrates the notion that modeling for components of a multicomponent object does not necessarily need to correlate, for each component, with conservation of angular momentum and conservation of linear momentum. This unit illustrates the notion that simple bosons can contribute any one of three symmetries regarding boost-related symmetry.

We explore dynamics modeling for components of hadron-like particles. (See discussion related to table 14.)

Table 44 reinterprets aspects of table 24. Each row in table 44 correlates with solutions that correlate with phenomena related to dynamics within hadron-like particles. For example, known hadron-like particles correlate with $1 \mathrm{Q} \otimes 2 \mathrm{U}$. Known hadron-like particles have internal interactions mediated by $2 \mathrm{U}$ simple particles, $2 \mathrm{~W}$ simple particles, and $2 \mathrm{G}$ long-range forces. Known hadron-like particles can emit $1 \mathrm{C}$ and $1 \mathrm{~N}$ particles. (The table does not list the $0 \mathrm{P}$ and $0 \mathrm{~K}$ solutions, which proposed theory suggests pertain to interactions between hadron-like particles but not necessarily to dynamics within hadron-like particles. See discussion regarding table 1.) Regarding table 44, each pairing of a boson solution with a fermion solution exhibits each of CP3, CA3, and a choice between B3, B2, and B0. CP3 correlates with $S U(2): \pm 1$ and with, for the hadron-like particle, one of conservation of angular momentum and conservation of momentum. (Here, the number after the colon denotes a contribution to the relevant $\widehat{A}_{X A}^{A L G}$. See table 4.) Regarding symbols of the form \pm , for other than $\Sigma \mathrm{G} \Gamma$, the plus pertains to the extent that either $n_{T A 0}=0$ or $n_{S A 0}=0$ and minus pertains to the extent that either $n_{T A 0}=-1$ or $n_{S A 0}=-1$. (For other than $\Sigma G \Gamma$, there are no cases of mismatches between $n_{T A 0}$ and $n_{S A 0}$.) CPA correlates with $S U(2): \pm 1$ and with, for the hadron-like particle, the other one of conservation of angular momentum and conservation of momentum. CC2 correlates with $U(1): 0$ and with conservation of 
Table 44: Properties and interactions, with respect to hadron-like particles, for simple particles and long-range forces

\begin{tabular}{|c|c|c|c|c|c|c|c|c|c|c|c|}
\hline$\Sigma \Phi$ & $\sigma$ & $\mid \leftarrow$ & $\cdots$ & $\mathrm{TA}$ & $\cdots$ & $\rightarrow \mid$ & $\leftarrow$ & $\cdots$ & $\mathrm{SA}$ & $\cdots$ & $\rightarrow \mid$ \\
\hline $\begin{array}{c}\text { or } \\
\Sigma \Phi \Gamma\end{array}$ & & 8,7 & 6,5 & 4,3 & 2,1 & 0 & 0 & 1,2 & 3,4 & 5,6 & 7,8 \\
\hline $0 \mathrm{H}$ & +1 & & & CP3 & CC2 & 0 & B0 & B2 & B3 & $*$ & \\
\hline OI & +1 & & & B3 & B2 & B0 & 0 & $\mathrm{CC} 2$ & CP3 & * & \\
\hline $1 \mathrm{~N}$ & +1 & & CBN2 & CA3 & ECT2s & -1 & -1 & ECS2 & G3 & CLN2 & \\
\hline $1 \mathrm{C}$ & +1 & & CBN2 & CA3 & ECT2s & 0 & 0 & ECS2 & G3 & CLN2 & \\
\hline $1 \mathrm{R}$ & -1 & & CBN2 & CA3 & ECT2 & -1 & -1 & ECS2 & G3 & CLN2 & \\
\hline $1 \mathrm{Q}$ & -1 & & CBN2 & CA3 & ECT2 & 0 & 0 & ECS2 & G3 & CLN2 & \\
\hline $2 \mathrm{U}$ & -1 & & & B3 & B2 & B0 & -1 & $\mathrm{CC} 2$ & CP3 & * & \\
\hline $2 \mathrm{~W}$ & +1 & & & CP3 & & 0 & B0 & B2 & B3 & * & \\
\hline $2 \mathrm{~T}$ & -1 & & & B3 & B2 & B0 & 0 & & CP3 & * & \\
\hline$\Sigma \mathrm{G} \Gamma$ & +1 & & & CP3 & $\mathrm{CC} 2$ & 0 & B0 & B2 & B3 & $*$ & \\
\hline
\end{tabular}

charge. The choice between B3, B2, and B0 correlates with a choice of modeling for the kinematics of a hadron-like particle. B3 correlates with $S U(2): \pm 1$, with boost symmetry, and with modeling (for the hadron-like particle) correlating with special relativity. B2 correlates with $U(1): 0$. Each of a TA-side B0 and an SA-side B0 correlates with $n_{T A 0}=n_{S A 0}$; with $\chi_{(0,0),(-1,-1)}$ and, with respect to the elementary boson, with $\widehat{A}_{(T A 0, S A 0)}^{A L G}=0$; and, for the hadron-like particle, with $\kappa_{0,-1}$ (or, no symmetry). (See table 4.) In table 44, each entry in the TA4-and-TA3 column and each entry in the SA3-and-SA4 column correlates with $S U(2)$. In table 44, each entry in the TA2-and-TA1 column and each entry in the SA1-and-SA2 column correlates with $U(1)$. The symbol * correlates with a boson channel. (See discussion related to equation (48) and discussion related to equation (123).) CBN2 correlates with $U(1): 0$ and with somewhat conservation of baryon number. CLN2 correlates with $U(1): 0$ and with somewhat conservation of lepton number. Conservation of lepton number minus baryon number correlates with a combination of CBN2 and CLN2. G3 correlates with $S U(2): \pm 1$ and with generation. CA3 correlates with $S U(2): \pm 1$ and with somewhat conservation of generation. For each of some (but not all) bosons, CP3 correlates with somewhat conservation of fermion generation for interactions with fermions. Each of ECT2s, ECT2, and ECS2 correlates with $U(1): 0$. The pair ECT2s and ECS2 correlates with conservation of charge. The pair ECT2 and ECS2 correlates with conservation of charge. For each row in table 44, the combination of conservation of momentum and conservation of angular momentum (or, the combination of CP3 and CA3) does not pertain.

Table 44 correlates with the notion that, if such could exist in nature, a free-ranging 1Q or 1R particle would correlate, at least with respect to ongoing theory, with some (at least virtual) bosons. Proposed theory modeling regarding such a $1 \mathrm{Q}$ or $1 \mathrm{R}$ particle does not include both emitting an elementary boson and absorbing the same elementary boson or a successor to the same elementary boson. (See remarks related to table 26.) In proposed theory, the notion of free environment does not pertain for individual simple fermions for which $\sigma=-1$.

Proposed theory suggests that a hadron-like particle must include at least two (non-virtual) fermions for which $\sigma=-1$. (The notion of virtual correlates with ongoing theory. Aspects of proposed theory do not necessarily include the notion of virtual fermions.) In addition, per the example regarding $1 \mathrm{Q} \otimes 2 \mathrm{U}$ hadron-like particles, there is no requirement for $n_{S A 0}$ for the simple fermions to match $n_{S A 0}$ for the elementary bosons.

\subsection{Possible modeling for fissionable or bound-state multicomponent ob- jects}

This unit discusses aspects of proposed theory modeling regarding multicomponent objects.

For PDE modeling regarding a multicomponent object, equation (113) and the following concepts might pertain. In the equation, $P$ denotes the magnitude of momentum. $A_{S A}^{P D E}$ correlates with $\left(m c^{2}\right)^{2}+$ $(P c)^{2}+f_{S A} . \quad A_{T A}^{P D E}$ correlates with $E^{2}+f_{T A}$. Here, each one of $f_{S A}$ and $f_{T A}$ is nonnegative. For some applications, $f_{S A}>0$ correlates with a fissionable system and possibilities for decay. Note that, if $f_{T A}=0, E^{2}$ exceeds $\left(m c^{2}\right)^{2}+(P c)^{2}$. For example, $f_{S A}>0$ might correlate with models for beta decay via the weak interaction. For some applications, $f_{T A}>0$ correlates with bound states. Note that, if 
$f_{S A}=0, E^{2}$ is less than $\left(m c^{2}\right)^{2}+(P c)^{2}$. For example, $f_{T A}>0$ might correlate with models for the structure of atomic nuclei. We are uncertain as to the extent to which such modeling for multicomponent objects would provide perspective that ongoing theory does not provide.

$$
E^{2}+f_{T A}=A_{T A}^{P D E}=A_{S A}^{P D E}=\left(m c^{2}\right)^{2}+(P c)^{2}+f_{S A}
$$

\subsection{Modeling regarding refraction and similar effects}

This unit discusses some aspects of kinematics and dynamics for zerolike rest mass particles, under assumptions regarding how the particles interact with their surroundings. This unit discusses modeling for the refraction of light. This unit discusses modeling regarding the existence of neutrino oscillations.

We discuss some aspects of kinematics and dynamics for simple particles for which $n_{S A 0}=-1$ and for long-range forces (for which $n_{S A 0}=-1$ ). The equation $n_{S A 0}=-1$ correlates with the notion of zerolike rest mass.

We explore kinematics and dynamics in contexts in which a zerolike rest mass elementary particle interacts with its surroundings. Known examples include photons in refractive media and gluons in hadrons. Similar considerations might pertain for neutrinos. Generally, we consider that a zerolike rest mass elementary particle and its surroundings constitute the two components of a system. We focus on kinematics or dynamics for the zerolike rest mass particle. We do not necessarily focus on kinematics for the system or dynamics for the system.

Mathematically, there are four cases to consider. The case of $\sigma=+1$ and $n_{T A 0}=0$ pertains for (at least) long-range forces. The case of $\sigma=+1$ and $n_{T A 0}=-1$ pertains for neutrinos. The case of $\sigma=-1$ and $n_{T A 0}=-1$ pertains for (at least) gluons. The case of $\sigma=-1$ and $n_{T A 0}=0$ is not necessarily physics-relevant. (We do not predict the existence of simple particles for which $\sigma=-1$ and $n_{T A 0} \neq n_{S A 0}$. Generally, see table 24.)

Each of equations (114) and (115) offers, based on using the range $-1<n_{S A 0}<0$, a possible basis for kinematics modeling regarding the zero rest mass elementary particle. (We contrast $-1<n_{S A 0}<0$ with $n_{S A 0}<-1$. Uses of the expression $n_{S A 0}<-1$ pertain for spin-related symmetry applications, for some modeling regarding gluons, and not necessarily for other purposes. Regarding the spin-related symmetry applications, see table 17. Regarding the gluon-related modeling, see table 45.) Here, $E$ denotes energy, $\vec{P}$ denotes momentum, $\vec{v}$ denotes velocity, $<_{-}>$denotes the expected value of $\_, P^{2}=<\vec{P} \cdot \vec{P}>$, and $v^{2}=\langle\vec{v} \cdot \vec{v}>$. Here, double-entry bookkeeping pertains to models for which at least one of the TA-side set of harmonic oscillators and the SA-side set of harmonic oscillators is not necessarily isotropic.

$$
\begin{gathered}
n_{S A 0}=-c^{2} P^{2} / E^{2} \\
n_{S A 0}=-v^{2} / c^{2}
\end{gathered}
$$

For each of the three physics-relevant cases, each of equations (114) and (115) adds a positive amount to $A_{S A}^{A L G}$. For each of the three cases, we posit that, for each relevant oscillator, $-1 \leq n \leq 0$ pertains.

For the case of $\sigma=+1$ and $n_{T A 0}=0$, for each relevant TA-side oscillator, $n_{T A}=-0$. One cannot satisfy double-entry bookkeeping by adding to $A_{T A}^{A L G}$. Satisfying double-entry bookkeeping correlates with subtracting something positive from at least one of the SA-side oscillators that correlate with $S U(2)$ kinematics symmetries. Proposed theory correlates this subtracting with aspects of refraction. Ongoing theory correlates the expression $c / v$ (or, $\left(c^{2} / v^{2}\right)^{1 / 2}$ ) with the two-word term refractive index (or, with the three-word term index of refraction). This case correlates with refraction of light.

For the case of $\sigma=+1$ and $n_{T A 0}=-1$, for each relevant SA-side oscillator, $n_{S A_{-}}=-1$. One cannot satisfy double-entry bookkeeping by adding to $A_{S A}^{A L G}$. Satisfying double-entry bookkeeping correlates with adding something positive to at least one of the two TA-side oscillators that correlate with $S U(2)$ approximate conservation of generation symmetry or to at least one of the TA-side oscillators that correlate with conservation of energy symmetry. The case correlates with neutrino oscillations.

For the case of $\sigma=-1$ and $n_{T A 0}=-1$, discussion is not quite as straightforward as is discussion for the other two physics-relevant cases. Discussion related to table 45 and table 46 pertains regarding gluons. (See discussion related to equation (116).)

Each of the three relevant cases might point to opportunities to develop new modeling. People might try to express kinematics conservation laws in terms of combinations, across modeling for each of a few interacting particles, via harmonic oscillator math. People might try to develop parallels to ongoing theory equations that, for example, sum momenta. We choose not to pursue such possible opportunities. 
Table 45: 2U solutions

\begin{tabular}{ccccccc}
\hline Solution & TA6 & TA5 & TA0 & SA0 & SA3 & SA4 \\
\hline 2U60 & -2 & -1 & 0 & -1 & -1 & -1 \\
2U56 & 0 & -2 & -1 & -1 & -1 & -1 \\
2U05 & -1 & 0 & -2 & -1 & -1 & -1 \\
2U50 & -1 & -2 & 0 & -1 & -1 & -1 \\
2U06 & 0 & -1 & -2 & -1 & -1 & -1 \\
2U65 & -2 & 0 & -1 & -1 & -1 & -1 \\
\hline
\end{tabular}

Table 46: 2U erase or paint ground states

\begin{tabular}{ccccccc}
\hline Ground state & TA6 & TA5 & TA0 & SA0 & SA3 & SA4 \\
\hline $2 \mathrm{U} 0=2 \mathrm{U} 60 \oplus 2 \mathrm{U} 50$ & -1 & -1 & 0 & 0 & -1 & -1 \\
$2 \mathrm{U} 6=2 \mathrm{U} 56 \oplus 2 \mathrm{U} 06$ & 0 & -1 & -1 & 0 & -1 & -1 \\
$2 \mathrm{U} 5=2 \mathrm{U} 05 \oplus 2 \mathrm{U} 65$ & -1 & 0 & -1 & 0 & -1 & -1 \\
\hline
\end{tabular}

We choose not to explore the extent to which modeling might feature mathematics for which at least one of the TA-side harmonic oscillator and the SA-side harmonic oscillator is not isotropic.

\subsection{U-family interactions and the strong interaction $S U(3)$ symmetry}

This unit discusses aspects regarding modeling gluons and modeling U-family interactions.

The $2 \mathrm{U}$ solutions correlate with gluons. Here, we provide details correlating with the TA-side $\kappa_{-1,-1,-1}$ symmetry that table $13 \mathrm{~b}$ shows.

Table 45 shows details regarding $2 \mathrm{U}$ solutions. The expression $\kappa_{-1,-1,-1}$ correlates with $A_{T A}^{A L G}=$ $-3 / 2$. Each one of the six TA-side $\pi_{0,-1,-2}$ permutations pertains. Each permutation correlates with $A_{T A}^{A L G}=-3 / 2$. Table 45 suggests notation for gluon-related solutions. The set of three permutations for which $0,-1$, and -2 appear in cyclic order correlates with interactions with one of matter simple fermions for which $\sigma=-1$ and antimatter simple fermions for which $\sigma=-1$. The set of the other three permutations correlates with the other choice between antimatter simple fermions for which $\sigma=-1$ and matter simple fermions for which $\sigma=-1$. Regarding matter simple fermions for which $\sigma=-1$, each of oscillators TA6, TA5, and TA0 correlates with a color charge. Relative to an ongoing theory standard representation for gluons, one of TA6 and TA5 correlates with the color red, the other of TA6 and TA5 correlates with the color blue, and TA0 correlates with the color green.

Ongoing theory correlates gluons with zero mass and with phenomena that proposed theory correlates with $2 \mathrm{U}$ solutions. We consider $2 \mathrm{U}$ phenomena regarding dynamics inside hadron-like particles. In such a frame of reference, proposed theory modeling based on equations (116) and (117) pertains. (Perhaps, compare with discussion, pertaining to refraction, regarding equations (114) and (115).) Here, the notation $a \leftarrow b$ correlates with the three-element phrase $a$ becomes $b$ (or, with the notion that $b$ replaces $a$ ). Here, the symbol $\rightarrow$ denotes, in the mathematical sense of a limit, the two-word phrase goes to.

$$
\begin{gathered}
\left(n_{S A 0}=-1\right) \leftarrow\left(n_{S A 0}=-v^{2} / c^{2} \rightarrow 0^{-}\right) \\
\left(n_{T A_{-}}=-2\right) \leftarrow\left(n_{T A_{-}}=\left(-1-v^{2} / c^{2}\right) \rightarrow(-1)^{-}\right)
\end{gathered}
$$

Equations (116) and (117) correlate with boson behavior for gluons. In effect, modeling of excitations and de-excitations correlates with a ground state that correlates with equation (118) and with, for the appropriate $n_{T A_{-}}$, equation (119). (See tables 45 and 46.) Excitation correlates with erasing a color charge (from, for example, a quark) and de-excitation correlates with painting a color charge (on, for example, a quark). (See discussion related to table 45.)

$$
\begin{aligned}
& n_{S A 0}=0 \\
& n_{T A_{-}}=0
\end{aligned}
$$

Table 46 shows results of applying, to items in table 45, aspects correlating with equations (118) and (119). Table 46 shows three erase or paint ground states. 
A gluon correlates with a weighted sum of two or three erase-and-paint pairs. For each pair, the erase part correlates with, in effect, an ability to erase, from the $\sigma=-1$ simple fermion that absorbs the gluon, a color. The paint part correlates with, in effect, an ability to paint, on to the $\sigma=-1$ simple fermion that absorbs the gluon, a color. The value $n_{T A}=0$ denotes an ability for a gluon to erase or paint the color charge correlating with the TA_ oscillator. Equation (120) shows an ongoing theory representation for one of the eight gluons. (Out of the eight gluons, this is the only one that involves three erase-and-paint pairs. Each of the other seven gluons involves two erase-and-paint pairs.) Regarding table 46, we make the following correlations. (Alternatively, without loss of generality or results, one might reverse the roles of TA6 and TA5.) The symbol $r$ correlates with painting the color red and with a painting application of 2 U6. The symbol $\bar{r}$ correlates with erasing the color red and with an erasing application of 2U6. The symbol $b$ correlates with painting the color blue and with a painting application of $2 \mathrm{U} 5$. The symbol $\bar{b}$ correlates with erasing the color blue and with an erasing application of $2 \mathrm{U} 5$. The symbol $g$ correlates with painting the color green and with a painting application of $2 \mathrm{U} 0$. The symbol $\bar{g}$ correlates with erasing the color green and with an erasing application of $2 \mathrm{U} 0$.

$$
(r \bar{r}+b \bar{b}-2 g \bar{g}) /(6)^{1 / 2}
$$

Ongoing theory correlates an $S U(3)$ symmetry with gluons. Proposed theory embraces the same $S U(3)$ symmetry per discussion nearby above. A $\kappa_{-1,-1}$ symmetry that correlates, in table $13 \mathrm{~b}$, with the oscillator pair SA3-and-SA4 reappears in table 46. This $\kappa_{-1,-1}$ symmetry correlates with conservation of fermion generation for interactions mediated by gluons.

\section{Relationships between various theories and models for objects}

This unit discusses relationships between various theories and models that pertain regarding objects. This unit discusses possible synergies between proposed theory and the elementary particle Standard Model. This unit suggests insight, that proposed theory might provide, regarding the strong CP problem and regarding axions. This unit discusses aspects regarding G-family interactions and channels. This unit suggests modeling that would comport with the notion that nature does not include the following - an elementary particle magnetic monopole, a non-zero electric dipole moment for any elementary particle, and a non-zero neutron electric dipole moment. This unit notes that proposed theory is not necessarily compatible with supersymmetry. This unit notes that aspects of proposed theory might help people explore the relevance of string theory to elementary particle physics. This unit explores concepts related to the masses of hadron-like particles that include arc simple fermions. This unit explores possibly useful modeling regarding the masses of quarks and charged leptons. This unit lists topics, regarding aspects of the cosmology timeline, for which proposed theory suggests insights. This unit shows a possible link between dynamics modeling that we suggest and a notion of entropy.

\subsection{The elementary particle Standard Model}

This unit discusses possible synergies between proposed theory and the elementary particle Standard Model.

People might try to add to the Standard Model some of the symmetries that proposed theory suggests. Examples include conservation of charge, approximate conservation of fermion generation, and approximate conservation of lepton number.

We discuss adding to the Standard Model some of the simple particles and long-range forces that proposed theory suggests.

To the extent that satisfying symmetries such as $S U(3) \times S U(2) \times U(1)$ boson symmetries suffices, people might be able to add, to the Standard Model, simple particles and long-range forces that proposed theory suggests.

Proposed theory might provide a basis for extending the Standard Model to include concepts related to mass and to forces that correlate with bosons that have spins of at least two.

People might explore synergies between Standard Model approaches and proposed theory approaches to various topics. One such topic is anomalous magnetic dipole moments.

We do not speculate regarding the extent to which people might find synergies between Lagrangian aspects of the Standard Model, models such as discussion related to refraction suggests, and kinematics conservation laws. (Regarding refraction, see discussion related to equation (115).) 


\subsection{The strong CP problem and possible axion elementary particles}

This unit suggests insight, that proposed theory might provide, regarding the strong CP problem and regarding axions.

Ongoing theory explores the possibility that the strong interaction contributes to violation of $\mathrm{CP}$ symmetry (or, charge conjugation parity symmetry). People might have yet to detect such violation. People use the three-element term strong CP problem. Theoretically, such violation might correlate with the existence of axions.

Each of the following statements might point to insight regarding the strong CP problem or regarding attempting to detect axions. Proposed theory suggests possible insight regarding CPT-related symmetries. (See table 50.) Proposed theory suggests insight regarding the electric dipole moment of the neutron. (See discussion related to equation (125).) Proposed theory suggests possible insight regarding the possible existence of magnetic monopoles. (See discussion related to equation (124).) Proposed theory suggests the possibility that people might mistake observations of phenomena related to the difference between 2(6)G248 and 2(1)G248 for observations related to axions. (See discussion related to equation (104).) Proposed theory suggests the possibility that people might mistake observations of phenomena related to the aye (or, 0I) boson for observations related to axions. (For example, equation (96) shows an interaction that might create effects that people might interpret as correlating with producing a magnetic field.)

To the extent that nature exhibits the relevant ongoing theory suggestion for non-zero CP violation, proposed theory suggests that some of the following statements might pertain. Table 50 points to TAside aspects that correlate with the non-conservation of CP symmetry. That non-conservation might correlate with breaking a possible $\pi_{r, b, g}$ symmetry correlating with red, blue, and green color charges. (See discussion related to equation (142).) That non-conservation might correlate with breaking an $S U(5)$ symmetry that correlates with conservation of energy. (See discussion related to equation (103).) Conservation of energy might pertain only to the extent that one includes consideration for at least two isomers of the universe that correlates with the relevant PRnISe modeling.

\subsection{Channels and G-family interactions}

This unit discusses aspects regarding G-family interactions and channels.

The notion of channels pertains to, for example, the relative strengths of electromagnetism and gravity. (See discussion related to equation (49).)

Each of equation (121) and equation (122) provides a candidate formula for the number of channels that pertain for the G-family solution $\Sigma$ G . Equation (121) correlates with table 11. Possible long-range forces correlating with $\Sigma \geq 10$ would not be relevant to physics. (See table 1.) Equation (122) correlates with table 10. Possible long-range forces correlating with $\Sigma \geq 10$ could be relevant to physics. We might not have enough data or theory to choose between candidate formulas.

$$
\begin{aligned}
& 5-(\Sigma / 2) \\
& 5-\left(\lambda_{\max } / 2\right), \text { with } \lambda_{\max }=\max \{\lambda \mid \lambda \in \Gamma\}
\end{aligned}
$$

From a standpoint of modeling, equation (122) might correlate with equation (17). Equation (122) might correlate with equation (18). To the extent that the correlations are relevant, equation (122) correlates with quantum transitions and equation (122) correlates with kinematics. These correlations might suggest that equation (122) correlates with nature and that G-family solutions for which $\Sigma \geq 10$ do not correlate with long-range forces.

Proposed theory suggests that each channel can correlate with a unique blank (or, $\left.\kappa_{0,-1}\right)$ SA-side oscillator pair in the range from SA3-and-SA4 through SA9-and-SA10. For this purpose, isotropic weighting pertains regarding oscillator pairs.

We discuss possible aspects of modeling for a $1 \mathrm{f} 1 \mathrm{~b} \rightarrow 1 \mathrm{f} 0 \mathrm{~b}$ interaction. The following notions pertain.

The incoming state de-excites by transferring one unit of $1 \mathrm{~b}$ excitation to one of the channels. For that channel, equation (123) pertains.

$$
\kappa_{0,-1} \rightarrow \kappa_{0,0}
$$

The new SA-side $S U(2)$ symmetry adds an extra kinematics-conservation-like symmetry that cannot last. (See table 14.) The interaction includes converting the $\kappa_{0,0}$ symmetry to something, pertaining to the outgoing state, such as $\kappa_{0,-1}$. (Discussion above de-emphasizes the notion that, for each SA-side 
channel, one TA-side channel exists. Double-entry bookkeeping suggests such a notion. An interaction would feature both a TA-side application of equation (123) and an SA-side application of equation (123). We think that the notion does not adversely impact results to which we allude.)

The above modeling is not incompatible with various proposed theory concepts, including the equal strengths of channels and the linear scaling, by number of channels, of interaction strengths.

\subsection{Lack of magnetic monopoles and a possible lack of some electric dipole moments}

This unit suggests modeling that would comport with the notion that nature does not include the following - an elementary particle magnetic monopole, a non-zero electric dipole moment for any elementary particle, and a non-zero neutron electric dipole moment.

Table 39 points to no G-family solutions that would correlate with interactions with a magnetic monopole elementary particle. The lack of such G-family solutions might correlate with nature not including a magnetic monopole elementary particle. People might want to consider the notion that equation (124) expresses.

The 2G2 solution correlates with electromagnetic (not magnetic) monopole moments.

Table 39 points to no G-family solutions that would correlate with a non-zero electric dipole moment for a point-like elementary particle. The lack of such G-family solutions might correlate with nature not including elementary particles that have non-zero electric dipole moments.

For each hadron for which modeling based on PDE techniques pertains and for which all the quarks occupy one state with respect to spatial characteristics, the electric dipole moment might be zero. (See discussion, related to table 25, regarding PDE-based modeling that correlates with some aspects of the strong, electromagnetic, and weak interactions.) Equation (125) shows an upper bound on the electric dipole moment for the neutron. (See reference [17]. Here, the one-letter symbol $\mathrm{m}$ denotes meters.) Proposed theory suggests that the neutron and proton might be such hadrons. Some research suggests that some pentaquarks might not be such hadrons. (See interpretation, in reference [73], of reference [76].)

$$
0.30 \times 10^{-27}\left|q_{e}\right| \mathrm{m}
$$

\subsection{Supersymmetry}

This unit notes that proposed theory is not necessarily compatible with supersymmetry.

Table 1 might suggest possibilities for some notion of symmetry based on equation (126). Here, the symbol $\leftrightarrow$ correlates with notions of exchanging roles. Table 1 might suggest possibilities for some notion of symmetry based on equation (127). For some relevant nonnegative values of $\Sigma$, table 1 might not suggest possibilities for a relevant notion of symmetry based on equation (128).

$$
\begin{aligned}
& \sigma=+1 \leftrightarrow \sigma=-1 \\
& m>0 \leftrightarrow m \doteq 0 \\
& \Sigma \leftrightarrow \Sigma+1
\end{aligned}
$$

Tables 1 and 39 seem, in themselves, to be incompatible with supersymmetry. People might explore the notion of layering supersymmetry over results that tables 1 and 39 show. However, given aspects of proposed theory, supersymmetry might not be necessary to explain known phenomena.

\subsection{String theory}

This unit notes that aspects of proposed theory might help people explore the relevance of string theory to elementary particle physics.

String theory correlates with notions of space-time frothiness on the scale of the Planck length (or, $R_{2}(m)$ ). (See equation (135).) Proposed theory suggests that there might be no need to appeal to such frothiness in order to limit sums of boson ground state energies. Leaving aside some mathematical aspects 
of proposed theory, proposed theory might not necessarily require that elementary particles have zero size. The Planck length might correlate with a size for elementary particles that have non-zero spin. (See equation (135).) The Schwarzschild radius might correlate with a size for elementary particles that have zero spin. (See equation (134).) Speculatively, the disparity between these two sizes might lead to means to explore making string theory more relevant to elementary particle physics that it has proven to be.

We suggest perspective about string theory and about proposed theory. (Reference [77] provides perspective about string theory.) Regarding simple particles and long-range forces, proposed theory correlates with the three-word term theory of what. Proposed theory outputs a list of what elementary particles nature embraces or might embrace. We contrast notions of theory of what with notions correlating with the three-word term theory of how. Proposed theory might not yet suggest a theory of how nature selects or forms elementary particles. Attempts to apply string theory might correlate with trying to develop a theory of how and trying to use the theory of how to produce a theory of what.

\subsection{Other discussion regarding the masses of simple particles and hadron-like particles}

This unit explores concepts related to the masses of hadron-like particles that include arc simple fermions. This unit explores possibly useful modeling regarding the masses of quarks and charged leptons.

We discuss rest energies for $1 \mathrm{R} \otimes 2 \mathrm{U}$ hadron-like particles.

The rest energy of a proton does not differ much from the rest energy of a neutron. For hadrons composed of generation-one quarks, the masses of hadrons do not vary much based on the masses of the quarks or on the charges of the quarks. The rest energies of $1 \mathrm{R} \otimes 2 \mathrm{U}$ hadron-like particles that contain exactly three arcs might approximate the rest energy of the proton, which is about $938 \mathrm{MeV}$. (Reference [17] provides data regarding hadron masses.) The rest energies of $1 \mathrm{R} \otimes 2 \mathrm{U}$ hadron-like particles that contain exactly two arcs might approximate the rest energy of the zero-charge pion, which is about 135 $\mathrm{MeV}$.

We explore another concept for estimating masses for $1 \mathrm{R} \otimes 2 \mathrm{U}$ hadron-like particles. The concept has bases in the relative densities of the universe of $1 \mathrm{Q} \otimes 2 \mathrm{U}$ hadrons and $1 \mathrm{R} \otimes 2 \mathrm{U}$ hadron-like particles.

Nature might have created concurrently, essentially, the current populations of $1 \mathrm{Q} \otimes 2 \mathrm{U}$ hadrons and $1 \mathrm{R} \otimes 2 \mathrm{U}$ hadron-like particles. We assume that each of $1 \mathrm{Q} \otimes 2 \mathrm{U}$ hadrons and $1 \mathrm{R} \otimes 2 \mathrm{U}$ hadron-like particles consists mainly of three-fermion particles. We explore three cases, in which, respectively, the span, s, of $1 \mathrm{R} \otimes 2 \mathrm{U}$ is one, six, and 36 . The respective spans correlate with PR1ISe modeling, PR6ISe modeling, and PR36ISe modeling. Equation (129) might estimate the current relevant ratio of density of $1 \mathrm{R} \otimes 2 \mathrm{U}$ hadron-like particles to density of ordinary matter. The symbol $m$ denotes the rest mass of a typical hadron-like particle. The left-most use of the ratio $m_{1 \mathrm{R} \otimes 2 \mathrm{U}} / m_{1 \mathrm{Q} \otimes 2 \mathrm{U}}$ correlates with rest energy (or rest mass) per particle. The right-most use of the ratio $m_{1 \mathrm{R} \otimes 2 \mathrm{U}} / m_{1 \mathrm{Q} \otimes 2 \mathrm{U}}$ occurs as the input to a calculation of an exponential and correlates with a hypothesis regarding the relative number of particles that nature created.

$$
\Omega_{\mathrm{ib}} /\left(\mathrm{s} \cdot \Omega_{\mathrm{b}}\right) \sim\left(m_{1 \mathrm{R} \otimes 2 \mathrm{U}} / m_{1 \mathrm{Q} \otimes 2 \mathrm{U}}\right) \exp \left(-m_{1 \mathrm{R} \otimes 2 \mathrm{U}} / m_{1 \mathrm{Q} \otimes 2 \mathrm{U}}\right)
$$

The respective values of $\Omega_{\mathrm{ib}} /\left(\mathrm{s} \cdot \Omega_{\mathrm{b}}\right)$ are $\sim 0.33, \sim 0.054$, and $\sim 0.0090$. For each value of $\mathrm{s}$, two mathematical solutions exist. The respective solutions, expressed in terms of $m \_c^{2}$ and in units of $\mathrm{GeV}$ are $\sim 0.6$ and $\sim 1.5, \sim 0.06$ and $\sim 4.4$, and $\sim 0.009$ and $\sim 6.6$. The long half-life of the proton might suggest that the rest energies of $1 \mathrm{R} \otimes 2 \mathrm{U}$ hadron-like particles are not less than the rest energy of the proton.

Table 47 summarizes some possible rest energies for $1 \mathrm{R} \otimes 2 \mathrm{U}$ hadron-like particles.

We explore concepts related to the lack of equality in equation (130). (See table 31 and equation (55).) Doing so might lead to insight about the term $\left(j_{M^{\prime \prime}}^{\prime \prime}\right) d^{\prime \prime}$ in equation (55). For the electron, muon, and tauon, the respective values of $M^{\prime \prime}$ are zero, two, and three. Regarding equation (130), a notion of equality - instead of inequality - would correlate with mathematical equality regarding the three values of $\log \left(m_{M^{\prime \prime}+1}\right)-\log \left(m_{M^{\prime \prime}}\right)$ for which $0 \leq M^{\prime \prime} \leq 2$. Here, $m_{M^{\prime \prime}}$ equals $m\left(M^{\prime \prime}, 3\right)$. (See equation (55).)

$$
m_{\mu}^{2} / m_{e}^{2}<\left(m_{\tau}^{2} / m_{\mu}^{2}\right)^{2}
$$

We determine a quantity $\omega_{e}$ that has units of mass; that might correlate mathematically, but not physically, with $6 \mathrm{G} 6$ or $8 \mathrm{G} 8$ strength related to all three charged leptons; and that satisfies equation (131). (Each of 6G6 and 8G8 does not interact with individual simple fermions and might not interact significantly with multicomponent objects.) The result $\omega_{e} \approx 0.3486 \mathrm{MeV} / c^{2}$ pertains. This result is 
Table 47: Possible rest energies for $1 \mathrm{R} \otimes 2 \mathrm{U}$ hadron-like particles

Possible rest energies $($ in $\mathrm{GeV})$ for $1 \mathrm{R} \otimes 2 \mathrm{U}$
1. $\sim 0.009$.
2. $\sim 0.06$.
3. $\sim 0.6$.
4. $\sim 1$.
5. $\sim 1.5$.
6. $\sim 4.4$
7. $\sim 6.6$.
8. Other.

somewhat less than the mass of the electron. This result does not necessarily comport with work regarding $\alpha^{2} m_{e}$. (See discussion regarding equation (20).) As yet, we do not find the exploration of $\omega_{e}$ to be physicsrelevant.

$$
\left(m_{\mu}^{2}-\omega_{e}^{2}\right) /\left(m_{e}^{2}-\omega_{e}^{2}\right)=\left(\left(m_{\tau}^{2}-\omega_{e}^{2}\right) /\left(m_{\mu}^{2}-\omega_{e}^{2}\right)\right)^{2}
$$

We explore a similar concept regarding quarks. Equation (132) pertains. (See equation (55).) The result $\omega_{q} \approx 3.02 \mathrm{MeV} / c^{2}$ pertains. This result might be somewhat less than the geometric mean of the experimental masses of the up and down quarks. (See table 31. Regarding equation (132), the notion of $m\left(M^{\prime \prime}, 3 / 2\right)$ correlates with the factor of $3 / 2$ that appears in equation (55) and with the notion that $\left.j_{3 / 2}^{\prime}=0.\right)$

$$
\left((m(1,3 / 2))^{2}+\omega_{q}^{2}\right) /\left((m(0,3 / 2))^{2}+\omega_{q}^{2}\right)=\left((m(2,3 / 2))^{2}+\omega_{q}^{2}\right) /\left((m(1,3 / 2))^{2}+\omega_{q}^{2}\right)
$$

We are uncertain as to possible significance for the notion that each of $\omega_{e}$ and $\omega_{q}$ is somewhat similar to the masses of the respectively relevant generation-one simple fermions.

\subsection{The cosmology timeline}

This unit lists topics, regarding aspects of the cosmology timeline, for which proposed theory suggests insights.

Work that we discuss above makes suggestions about the following aspects of the ongoing theory cosmology timeline.

- Inflation.

- Phenomena just after inflation.

- The production of baryon asymmetry.

- Eras regarding the rate of expansion of the universe.

- Dark matter baryon-like acoustic oscillations, plus effects of those acoustic oscillations that lead to at least some aspects of filaments.

- Clumping that forms various objects, such as stars and galaxies.

- Galaxy formation and evolution.

Proposed theory suggests the following notions. 
Table 48: Some phenomena that people might want to feature in cosmology timelines

Phenomena

- Transition to dominance by left-handed simple fermions. (See discussion related to equation (103).)

- Production of $1 \mathrm{R} \otimes 2 \mathrm{U}$ hadron-like particles. (Possibly, the vanishing of seas composed of gluons and quarks or arcs.)

- Transition in dominance, regarding various sizes of objects, from repulsion based on 4(1)G2468a and 4(1)G2468b to attraction based on 4(1)G246. (See discussion related to table 41.)

- Earliest visible galaxies of various types that table 42 suggests.

- Achievement, by some galaxies, of approximately four to one ratios of dark matter density to ordinary matter density. (See table 42.)

- Transition in dominance, regarding various sizes of objects, from attraction based on 4(1)G246 to repulsion based on 4(2)G48. (See discussion related to table 41.)

- Transition in dominance, regarding various sizes of objects, from repulsion based on 4(2)G48 to attraction based on 4(6)G4. (See discussion related to table 41.)

- Scenarios regarding clumping suggest that a significant fraction of early black holes contained stuff correlating with essentially just one isomer of PR6ISe-span-one phenomena. Approximately onesixth of such one-isomer black holes correlates with each one of the six isomers of PR6ISe-span-one phenomena.

- Aspects of quasars, black hole jets, blazars, and eruptions from supermassive black holes might correlate with effects of the 4G48 repulsive long-range force. Similarly, aspects of radio-wave emitting bubbles might correlate with effects of the $4 \mathrm{G} 48$ repulsive long-range force. (See references [78] and [79].)

- Aspects of black hole or neutron star collisions might correlate with effects of the $4 \mathrm{G} 48$ repulsive long-range force.

Proposed theory also might suggest the following notions.

- Early in the evolution of the universe, the universe that people know might have decoupled from, destroyed, or expelled right-handed simple fermions. (See discussion related to equation (103).)

- Early in the evolution of the universe, quarks, arcs, and gluons formed hadron-like seas. The seas might have undergone phase changes, with the last changes featuring at least one transition from seas to hadron-like particles.

- Proposed theory is not incompatible with possible large-scale flatness for the universe.

Table 48 suggests some phenomena that people might want to feature in cosmology timelines.

\subsection{Entropy}

This unit shows a possible link between dynamics modeling that we suggest and a notion of entropy.

We consider cases of multicomponent objects that involve $k+1$ peer component objects. Here, $k$ is a nonnegative integer.

We consider the case of $k=1$. The multicomponent object includes two peer component objects. Compared with dynamics symmetries for the multicomponent object, the two peer components collectively contribute one too many instance of each of conservation of energy symmetry, conservation of angular momentum symmetry, and conservation of momentum symmetry. Modeling can re-assign the extra three symmetries to a field - such as a gravitational field - that correlates with interactions between the peer components.

We consider the case of $k>1$. Here, we de-emphasize the possibility of non-peer subdivision. An example of non-peer subdivision involves the sun, earth, and moon. For this example of non-peer subdivision, one might use two steps, each correlating with $k=1$. The first step considers each of the sun and the earth plus moon to be objects. The second step considers the earth plus moon to be a multicomponent object consisting of the earth and the moon. Without adequately significant additions to modeling, this example might correlate with modeling for which - regarding ocean tides - effects of lunar gravity pertain and effects of solar gravity do not pertain. 
For $k>1$, ongoing theory modeling becomes more complex than ongoing theory modeling for twobody (or, $k=1$ ) systems. Many applications might pertain - for example, to astrophysical systems, to ideal gasses, and so forth. For some applications, keeping the number of fields at one might correlate with a notion of entropy and, at least within that notion, with the ongoing theory expression for entropy that equation (133) shows. Here, people might want to consider at least one of the two cases $j=k+1$ and $j=k$. Here, people might want to consider each of a notion of entropy for physical systems and a notion that might correlate, regarding mathematics-based modeling, with a term correlating with the word entropy.

$$
j \log (j)
$$

\section{Possible opportunities to develop deeper insight}

This unit discusses modeling that people might use to develop new aspects of physics theory.

\subsection{Numbers of dimensions}

This unit speculates regarding one aspect of the topic of numbers of dimensions.

Proposed theory suggests that, at least in some sense, a number - three - of spatial dimensions correlates with $D_{S A}^{*}=3$ and a number - one - of temporal dimensions correlates with $D_{T A}^{*}=1$. (See equations (34) and (35).)

For a hypothetical five spatial dimensions and $D_{S A}^{*}=5$, for a simple fermion, the particle might correlate with $\nu_{S A}=-5 / 2$ and modeling might suggest relevance for two fields. One field could correlate with $\nu_{S A}=-1 / 2$. One field could correlate with $\nu_{S A}=-3 / 2$. The notion of two fields might correlate with a lack of physics relevance.

\subsection{A series of formulas for lengths, including the Planck length}

This unit discusses three related formulas that produce lengths.

We suggest a series of formulas for lengths. Equation (134) correlates with the Schwarzschild radius for an object of mass $m$. Equation (135) correlates with the Planck length and does not depend on $m$. Equation (136) includes a factor of $m^{-1}$. When applied to the mass of $2 \mathrm{~W}$ bosons, equation (136) correlates somewhat with the range of the weak interaction. When applied to the mass of a charged pion, equation (136) correlates somewhat with a range for the component, of the strong interaction, that has bases in gluons. Equation (137) shows the ratio between successive formulas. Equation (138) shows, for the electron, the ratio correlating with equation (137).

$$
\begin{gathered}
R_{4}(m)=\left(G_{N}\right)^{1} m^{1} \hbar^{0} c^{-2} 2^{1} \\
R_{2}(m)=\left(G_{N}\right)^{1 / 2} m^{0} \hbar^{1 / 2} c^{-3 / 2} 2^{0} \\
R_{0}(m)=\left(G_{N}\right)^{0} m^{-1} \hbar^{1} c^{-1} 2^{-1} \\
\left(G_{N}\right)^{-1 / 2} m^{-1} \hbar^{1 / 2} c^{1 / 2} 2^{-1} \\
\left(G_{N}\right)^{-1 / 2}\left(m_{e}\right)^{-1} \hbar^{1 / 2} c^{1 / 2} 2^{-1} \approx 1.1945 \times 10^{22}
\end{gathered}
$$

Proposed theory might point to $R_{0}\left(m_{H^{0}}\right)$ as being a minimal size relevant for some modeling of aspects of objects that contain more than one simple fermion. (Here, $m_{H^{0}}$ denotes the mass of the Higgs boson.) 
Table 49: TSP, APM, and SSP transformations (regarding ALG models)

\begin{tabular}{|c|c|c|c|c|}
\hline $\begin{array}{c}\text { Swap } \\
\text { (for each odd } j^{\prime} \\
\text { and }\end{array}$ & Swap & \multicolumn{3}{|c|}{$\begin{array}{l}\text { Swap pertains } \\
\text { for the } \\
\text { transformation }\end{array}$} \\
\hline with $\left.j^{\prime \prime}=j^{\prime}+1\right)$ & & TSP & APM & SSP \\
\hline$n_{T A j^{\prime \prime}}$ and $n_{T A j^{\prime}}$ & - & Yes & Yes & No \\
\hline- & $n_{T A 0}$ and $n_{S A 0}$ & No & No & No \\
\hline$n_{S A j^{\prime}}$ and $n_{S A j^{\prime \prime}}$ & - & No & Yes & Yes \\
\hline
\end{tabular}

Table 50: Ongoing theory T, C, and P transformations, in a context of proposed theory ALG models

\begin{tabular}{|c|c|c|c|c|c|c|c|}
\hline \multirow{2}{*}{$\begin{array}{c}\text { Swap } \\
\text { (for each odd } j^{\prime} \\
\text { and } \\
\text { with } j^{\prime \prime}=j^{\prime}+1 \text { ) }\end{array}$} & \multirow[t]{2}{*}{ Swap } & \multicolumn{3}{|c|}{$\begin{array}{l}\text { Swap pertains } \\
\text { for the } \\
\text { transformation }\end{array}$} & \multicolumn{3}{|c|}{$\begin{array}{l}\text { Transformation and } \\
\text { swap pertain for gluons } \\
\text { and color charge }\end{array}$} \\
\hline & & $\mathrm{T}$ & $\mathrm{C}$ & $\mathrm{P}$ & $\mathrm{T}$ & $\mathrm{C}$ & $\mathrm{P}$ \\
\hline$n_{T A j^{\prime \prime}}$ and $n_{T A j^{\prime}}$ & - & Yes & Yes & No & No & No & No \\
\hline- & $n_{T A 0}$ and $n_{S A 0}$ & No & No & No & No & No & No \\
\hline$n_{S A j^{\prime}}$ and $n_{S A j^{\prime \prime}}$ & - & No & Yes & Yes & No & No & No \\
\hline
\end{tabular}

\subsection{CPT-related symmetries}

This unit discusses some proposed theory symmetries and some aspects of ongoing theory CPT-related symmetries.

Table 49 summarizes proposed theory concepts regarding so-called TSP, APM, and SSP transformations. The table pertains for ALG models. TSP abbreviates the three-word phrase temporal side parity (or, TA-side parity). APM abbreviates the three-element phrase antiparticle or anti-mode. SSP abbreviates the three-word phrase spatial side parity (or, SA-side parity).

Ongoing theory includes notions of $\mathrm{C}$ (or, charge-reversal) transformation and approximate symmetry, $\mathrm{P}$ (or, parity-reversal) transformation and approximate symmetry, and $\mathrm{T}$ (or, time-reversal) transformation and approximate symmetry. In ongoing theory, invariance under CPT transformation pertains.

Table 50 might correlate with ongoing theory notions of T, C, and P approximate symmetries. Similarities exist between TSP transformation and $\mathrm{T}$ (or, time reversal) transformation, between APM transformation and $\mathrm{C}$ (or, charge-reversal) transformation, and between SSP transformation and P (or, parity-reversal) transformation. A significant difference between TSP symmetry and T symmetry and a significant difference between APM symmetry and C symmetry might pertain and might correlate with gluons and with color charge.

People might want to consider implications of the possibility that conservation of each of TSP, ASP, and SSP pertains more exactly than does conservation of (respectively) T, C, and P. This possibility might explain aspects of the strong CP problem. (Regarding CP violations, see, for example, reference [80].)

\subsection{Relationships between properties of objects and forces}

This unit notes that proposed theory might point to opportunities to further explore relationships between charge and mass. This unit notes that proposed theory might point to opportunities to further explore relationships between strengths of components of long-range forces. This unit suggests a possible opportunity to explore relationships between handedness, chirality, helicity, lepton number or baryon number, rotation, and spin.

We discuss relationships between charge and mass.

The fine-structure constant appears in a formula that links the masses of the six quarks to the masses of the three charged leptons. (See equation (55).) The fine-structure constant includes, as a factor, the square of the charge of the electron. (See equation (56).)

The charge of an electron and the mass of an electron appear in a formula that links the mass of a tauon to the mass of an electron. (See equation (49).)

Generally, the mass of a known charged hadron is less than the mass of a similar uncharged object. One example compares the mass of a charged pion to the mass of a neutral pion. One example compares the mass of a proton to the mass of a neutron. Modeling based on equation (113) might correlate a 
portion of $f_{T A}$ with non-zero charge.

The mass of the $\mathrm{W}$ boson is less than the mass of the $\mathrm{Z}$ boson. The square of the mass of the $\mathrm{W}$ boson is proportional to $10-1-2$. (See discussion related to equation (45).) The square of the mass of the $\mathrm{Z}$ boson is proportional to $10-1$. The two in the expression that is proportional to the square of the mass of the $\mathrm{W}$ boson seems to link charge and mass. The one or the ten in the expressions that are proportional to the squares of masses of the $\mathrm{W}$ or $\mathrm{Z}$ bosons might link spin and mass.

Proposed theory might suggest opportunities to further explore relationships between charge and mass and relationships between strengths of components of long-range forces.

Proposed theory might suggest another opportunity to explore modeling related to masses. We discuss a possibly useful notion regarding masses of non-zero-mass simple particles. Equations (139) and (140) pertain. The symbol $m$ denotes mass. Boson simple particle masses tend to feature relationships regarding squares of masses. Equation (139) points to results that feature squares of masses. For each simple $\sigma=+1$ boson, the equation evaluates approximately to an integer. The equation might correlate with the 2U-related potential that scales like $r^{1}$ and pertains regarding $\sigma=-1$ simple fermions in hadronlike particles. Equation (140) points to results that feature logarithms of masses. For each simple fermion other than the neutrinos, the equation evaluates somewhat approximately to an integer. The equation might correlate with $\Sigma$ G-related potentials that scale like $r^{-1}$ and pertain regarding simple fermions that have quantum interactions with $2 \mathrm{G} 2$ and 4G4 long-range forces. Equation (141) follows from equation (140) and produces results pertaining to squares of simple fermion masses other than neutrino masses.

$$
\begin{gathered}
\int_{0}^{m /\left(m_{Z} / 3\right)} 2 r^{1} d r \\
\int_{1}^{m / m_{e}} r^{-1} d r \\
\int_{1}^{m / m_{e}} 2 r^{-1} d r
\end{gathered}
$$

Proposed theory might offer an opportunity for new looks, regarding models, at relationships between handedness, chirality, helicity, lepton number or baryon number, rotation, and spin.

\subsection{Arrow of time and entropy}

This unit notes that proposed theory may provide perspective regarding the topic of arrow of time and regarding the topic of entropy.

We discuss aspects regarding arrow of time.

Equation (108) suggests a $\Psi(t, r)$ that correlates with the TA0-and-SA0 oscillator pair. (See equation (29).) The domains $t>0$ and $r>0$ pertain for $\Psi(t, r)$. Without loss of generality, we posit that $\eta_{T A}>0$ pertains regarding after an interaction, $\eta_{T A}>0$ does not pertain regarding before an interaction, $\eta_{T A}<0$ pertains regarding before an interaction, and $\eta_{T A}<0$ does not pertain regarding after an interaction. We posit that $\eta_{S A}>0$ pertains regarding elementary particles that exit an interaction, $\eta_{S A}>0$ does not pertain regarding elementary particles that enter an interaction, $\eta_{S A}<0$ pertains regarding elementary particles that enter an interaction, and $\eta_{S A}<0$ does not pertain regarding elementary particles that exit an interaction. Of the four possibilities $\eta_{T A}>0$ and $\eta_{S A}>0, \eta_{T A}<0$ and $\eta_{S A}<0, \eta_{T A}>0$ and $\eta_{S A}<0$, and $\eta_{T A}<0$ and $\eta_{S A}>0$, mathematically, $\Psi$ normalizes for only the first two possibilities. To the extent that this modeling correlates with the topic of arrow of time, the lack of dual normalization regarding each of the case of incoming and the case of outgoing might provide insight.

The proposed theory notion that modeling of conservation of energy correlates with an $S U(5)$ symmetry (and not necessarily with an ongoing theory notion of $S 1 G$ symmetry) might provide insight regarding the topic of arrow of time. Proposed theory tends to correlate $S U\left(_{-}\right)$symmetries with origins (with respect to coordinates) and with radial coordinates.

We discuss aspects regarding entropy.

Discussion related to equation (133) might provide insight regarding entropy.

Speculatively, interactions correlating with the 8G8 solution catalyze, in some situations, increases in entropy. (See discussions related to tables 14 and 40.) 


\subsection{The Higgs mechanism, entanglement, and tachyon-like behavior}

This unit provides possible proposed theory perspective regarding the ongoing theory notions of a Higgs mechanism, entanglement, and tachyon-like behavior.

At least to the extent that one models the universe as being a confined environment, the following statements might pertain.

- The aye (or, 0I) boson correlates with the Higgs mechanism or Higgs field.

- Theory does not completely disentangle any object from a notion of the universe minus that object.

- These notions correlate with a large-scale notion of tachyon-like behavior.

Proposed theory QFT includes volume-like interaction vertices. Especially to the extent that models correlate with $v_{c}>c$, people might interpret these vertices as correlating with tachyon-like behavior. (See discussions related to equation (40) and equation (44).)

\subsection{Notions regarding possible universes beyond our universe}

This unit speculates about some notions regarding possible universes beyond our universe.

Regarding the G-family, beyond modeling that includes models for channels, one might correlate the oscillator pairs SA11-and-SA12, SA13-and-SA14, and SA15-and-SA16 with conservation of angular momentum symmetry, conservation of linear momentum symmetry, and boost-related symmetry. (See discussion regarding equation (123).) Doing so features modeling that involves 17 SA-side oscillators and that might correlate with relevance for an $S U(17)$ symmetry. (Note remarks regarding equation (11).)

Within a context correlating with the symmetries that equation (142) shows, modeling for all known physics might correlate with a notion of confined environment and a notion that we might characterize by $\sigma_{17}=-1$. (Compare with $\sigma=-1 \mathrm{in}$, for example, table 24 . Regarding the possibility correlating with a TA-side $S U(7)$ symmetry, see table 44 and, perhaps, also note that one might, in table 24 , move information regarding the TA8-and-TA7 oscillator pair to the TA6-and-TA5 oscillator pair.)

TA-side: $S U(7)$ or $S U(17)$, SA-side: $S U(17)$

The ratio of the number of generators of $S U(17)$ to the number of generators of $S U(7)$ is six (or, 288/48). Regarding discussion regarding equation (142), this factor of six might correlate with a $\pi_{r, b, g}$ symmetry correlating with red, blue, and green color charges and with oscillators TA6, TA5, and TA0. (See table 45 and discussion related to equation (103).)

In the context of $\sigma_{17}=-1$, the factor of six might correlate with a $U(1)$ symmetry (for which the number of generators is two) and an $S U(2)$ symmetry (for which the number of generators is three). (See discussion related to equation (142).) Speculatively, one or more of the following notions might pertain.

- Our universe is one of either two or six universes in a so-called larger-scale universe that includes, respectively, two or six objects of the scale of our universe.

- A big bang for the larger-scale universe created, in effect, our universe and an anti-universe.

- An ongoing theory somewhat analog of a possible T-symmetry-related conservation of energy pertains across the creation of this our-universe and anti-universe pair.

- The notion of two or six such universes might extend to a notion of 24 such universes. (See discussion related to equation (103).)

\subsection{Notions that might link physics constants and modeling}

This unit speculates regarding relationships between some minimal non-zero values that people observe and some aspects of proposed theory.

Table 51 shows speculation about possible conflations regarding three notions. One notion is the $\Sigma$ in G-family mathematical solutions $\Sigma$ G $\Gamma$. One notion is quantities (or, properties) with which some $\Sigma \gamma$ components of G-family long-range forces interact. One notion is the $r^{n}$ potential that might correlate with PDE modeling for some aspects of the relevant G-family mathematical solution. (Such a potential might pertain regarding three dimensions and three oscillators. Such a potential might pertain regarding two dimensions and two oscillators.) Each quantity (or, property) might pertain for each of some aspects of classical physics modeling and some aspects of quantum physics modeling. (Compare with table 19 
Table 51: Possible conflations regarding G-family solutions, properties with which G-family forces interact, and aspects of PDE modeling (with suggested smallest non-zero property magnitudes regarding $\sigma=+1$ modeling)

\begin{tabular}{|c|c|c|c|c|c|}
\hline$\Sigma$ & Scalar & Vector & 2-tensor & 3 -tensor & PDE potential \\
\hline 0 & & & & & $r^{0}$ \\
\hline 2 & Charge $\left(\left|q_{e}\right|\right)$ & Magnetic flux $\left(K_{\mathrm{J}}\right)$ & Rotating flux & & $r^{-1}$ \\
\hline 4 & Mass & Rotating mass & Moments of inertia & Rotating moments & $r^{-1}$ \\
\hline 6 & & & & & $r^{+1}$ \\
\hline 8 & $N_{L-B}(1)$ & $\operatorname{Spin}(\hbar / 2)$ & & & $r^{-1}$ \\
\hline$\ldots$ & & & & & $r^{+1}$ \\
\hline 16 & Speed $(c)$ & & & & $r^{-1}$ \\
\hline
\end{tabular}

and table 21.) In table 51, an item in parentheses shows a non-zero magnitude that pertains for modeling that correlates with $\sigma=+1$. For charge, for $\sigma=-1,\left|q_{e}\right| / 3$ pertains. For lepton number minus baryon number, for $\sigma=-1,1 / 3$ pertains. Except for regarding speed, for either $\sigma=+1$ or $\sigma=-1$, the number is a minimal non-zero magnitude. Some modeling regarding refraction and effective mass might correlate (via, aspects correlating with longitudinal polarization) with a lack of a minimal non-zero quantity. (See discussion related to equations (114) and (115).) The rightmost column of table 51 speculates about relationships to equations such as equations (26) and (27). Equations (143), (144), and (145) may pertain. Table 51 does not show quantity centric information regarding rows for which a potential of other than $r^{-1}$ pertains. Regarding the case of $\Sigma=6$, a correlation between $r^{+1}$ and the association of aspects of the strong interaction with the oscillator pairs TA5-and-TA6 and SA5-and-SA6 might pertain. (See discussion related to table 13b.) Regarding the case of $\Sigma=16$, there might be a correlation with the notion that modeling might correlate boost symmetry with the oscillator pair SA15-and-SA16. (See discussion that includes discussion of table 15.) Such a correlation might dovetail with solutions that allow $\lambda=\llbracket 16 \rrbracket \in \Gamma$. Some items for which equation (145) pertains might correlate with aspects of kinematics. Some such items might correlate with one or more of $D^{\prime \prime}=2$ and $S U(2)$ symmetry. For example, one such $S U(2)$ symmetry might correlate with conservation of angular momentum.

$$
\begin{aligned}
& r^{0}, \text { for } \Sigma=0 \\
& r^{-1}, \text { for } \log _{2} \Sigma \text { being a positive integer } \\
& r^{+1}, \text { for } \log _{2} \Sigma \text { being a positive non-integer }
\end{aligned}
$$

Some items in table 51 might correlate, in essence, with other physics constants. Charge might correlate with $1 /\left(4 \pi \varepsilon_{0}\right)$ and the vacuum electric permittivity $\varepsilon_{0}$. Magnetic flux correlates with $\left|q_{e}\right|$ and $\hbar$ and might correlate with $\mu_{0}$, the vacuum magnetic permeability. Mass might correlate with $G_{N}$, the gravitational constant.

We are uncertain regarding the usefulness of pursuing further such notions.

\section{Concluding remarks}

This unit discusses possible opportunities based on proposed theory.

Proposed theory might provide impetus for people to tackle broad agendas that the work suggests. Proposed theory might provide means to fulfill aspects of such agendas. Proposed theory might fulfill aspects of such agendas.

Opportunities might exist to develop more sophisticated theory and modeling than the theory and modeling that we present. Such a new level of work might provide more insight than we provide.

Proposed theory might suggest - directly or indirectly - opportunities for observational research, experimental research, development of precision measuring techniques and data analysis techniques, numerical simulations, and theoretical research regarding elementary particle physics, nuclear physics, atomic physics, astrophysics, and cosmology.

Proposed theory might suggest applied mathematics techniques that have uses other than uses that we make. 


\section{Acknowledgments}

The following people pointed to topics or aspects that we considered for inclusion in the scope of the work: Andrea Albert, Raphael Bousso, Lance Dixon, Persis Drell, Alex Filippenko, Brian Greene, Kamal Melek Hanna, Wick Haxton, Nick Hutzler, Surhud More, Holger Muller, Martin Rees, and various science journalists. The following people helped formally publish aspects of the work: Charles K. Chui, Kamal Melek Hanna, Keith Jones, and Zeger Karssen. (See references [81] and [82].) The following people provided or pointed to aspects regarding expressing or propagating the work: Man Ho Chan, Maxwell Chertok, Charles K. Chui, Steven Frautschi, Carl Frederick, Richard B. Holmes, Juan Jose Lietor-Santos, Frank Hiroshi Ling, Michael Mulhearn, Richard A. Muller, Stephen Perrenod, Paul Preuss, Amir Sharif, and Wendy Shi. The following people suggested perspective, means, or suggestions regarding people with whom to try to have discussions: Yanbei Chen, Bill Daul, George Djorgovski, Vesselin Gueorguiev, Doug Osheroff, and Jon F. Wilkins. The following people provided means or encouragement relevant to this work: Helen Buckholtz, Joel Buckholtz, Sylvia Buckholtz, George Michael, and various teachers.

\section{References}

[1] Guillermo Ballesteros, Javier Redondo, Andreas Ringwald, and Carlos Tamarit. Several Problems in Particle Physics and Cosmology Solved in One SMASH. Frontiers in Astronomy and Space Sciences, 6, July 2019. 1.3

[2] D. Mendeleev. Ueber die beziehungen der eigneschaften zu den atomgewichten der elemente. Zeitschrift fur Chemie, 12:405-406, March 1869. 1.5.1

[3] L. Verde, T. Treu, and A.G. Riess. Tensions between the early and the late universe. July 2019. Link: https://arxiv.org/abs/1907.10625. 1.5 .2

[4] Johanna L. Miller. Gravitational-lensing measurements push hubble-constant discrepancy past $5 \sigma$. Physics Today, 2020(1):0210a, February 2020. 1.5.2

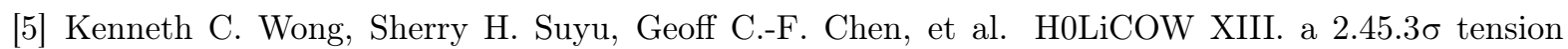
between early and late-Universe probes. Monthly Notices of the Royal Astronomical Society, in press 2020. Link: https://arxiv.org/abs/1907.04869. 1.5.2

[6] Natalie Wolchover. New Wrinkle Added to Cosmology's Hubble Crisis. Quanta Magazine, February 2020. Link: https://www.quantamagazine.org/new-wrinkle-added-to-cosmologys-hubble-crisis$20200226 / .1 .5 .2$

[7] Wendy L. Freedman, Barry F. Madore, Taylor Hoyt, et al. Calibration of the Tip of the Red Giant Branch (TRGB). February 2020. Link: https://arxiv.org/abs/2002.01550. 1.5 .2

[8] Vivian Poulin, Tristan L. Smith, Tanvi Karwal, and Marc Kamionkowski. Early dark energy can resolve the Hubble tension. Phys. Rev. Lett., 122:221301, June 2019. 1.5.2, 1.5.6, 4.8

[9] N. G. Busca, T. Delubac, J. Rich, et al. Baryon acoustic oscillations in the lya forest of boss quasars. Astronomy \& Astrophysics, 552(A96), April 2013. 1.5.2, 3.5

[10] S. Perlmutter, G. Aldering, G. Goldhaber, et al. Measurements of $\Omega$ and $\Lambda$ from 42 high-redshift supernovae $\Omega$. The Astrophysical Journal, 517(2):565-586, June 1999. 1.5.2, 3.5

[11] Adam G. Riess, Alexei V. Filippenko, Peter Challis, et al. Observational evidence from supernovae for an accelerating universe and a cosmological constant. The Astronomical Journal, 116(3):1009-1038, September 1998. 1.5.2, 3.5

[12] Adam G. Riess, Louis-Gregory Strolger, John Tonry, et al. Type ia supernova discoveries at z $>1$ from the Hubble Space Telescope: Evidence for past deceleration and constraints on dark energy evolution. The Astrophysical Journal, 607:665-687, June 2004. 1.5.2, 3.5

[13] Brian Green. Until the End of Time: Mind, Matter, and Our Search for Meaning in an Evolving Universe. Alfred A. Knopf, February 2020. 1.5.2, 3.6, 3.6, 4.8

[14] Motoi Endo, Koichi Hamaguchi, and Go Mishima. Constraints on hidden photon models from electron $g-2$ and hydrogen spectroscopy. Phys. Rev. D, 86:095029, Nov 2012. 1.5.3 
[15] Edward Witten. Cosmic separation of phases. Phys. Rev. D, 30:272-285, July 1984. 1.5.3

[16] M. H. Chan. Observational evidence for dark matter interacting through a Yukawa potential. The Astrophysical Journal, 769(1):L2, May 2013. 1.5.3

[17] M. Tanabashi and others (Particle Data Group). Review of particle physics. Phys. Rev. D, 98:030001, August 2018. 1.5.3, 1.5.7, 2.4, 2.4, 2.10, 2.11, 2.13, 2.15, 2.16, 3.3, 3.3, 3.10, 5.4, 5.7

[18] Pieter van Dokkum, Roberto Abraham, Jean Brodie, et al. A high stellar velocity dispersion and 100 globular clusters for the ultra-diffuse galaxy Dragonfly 44. The Astrophysical Journal Letters, 828(1):L6, 2016. http://iopscience.iop.org/article/10.3847/2041-8205/828/1/L6. 1.5.3, 3.8

[19] R. Genzel, N. M. Forster Schreiber, H. Ubler, et al. Strongly baryon-dominated disk galaxies at the peak of galaxy formation ten billion years ago. Nature, 543(7645):397-401, March 2017. 1.5.3, 3.8

[20] Peter Behroozi, Risa Wechsler, Andrew Hearin, and Charlie Conroy. UniverseMachine: The correlation between galaxy growth and dark matter halo assembly from $\mathrm{z}=0-10$. June 2018. Link: https://arxiv.org/abs/1806.07893v1. 1.5.3, 3.8

[21] J. Jimenez-Vicente, E. Mediavilla, C. S. Kochanek, and J. A. Munoz. Dark matter mass fraction in lens galaxies: New estimates from microlensing. The Astrophysical Journal, 799(2):149, 2015. 1.5.3, $3.8,3.8$

[22] David A. Buote and Aaron J. Barth. The extremely high dark matter halo concentration of the relic compact elliptical galaxy mrk 1216. The Astrophysical Journal, 877(2):91, May 2019. 1.5.3, 3.8

[23] Ana Bonaca, David W. Hogg, Adrian M. Price-Whelan, and Charlie Conroy. The Spur and the Gap in GD-1: Dynamical evidence for a dark substructure in the Milky Way halo. November 2018. Link: https://arxiv.org/abs/1811.03631. 1.5.3, 3.8

[24] Judd D. Bowman, Alan E. E. Rogers, Raul A. Monsalve, et al. An absorption profile centred at 78 megahertz in the sky-averaged spectrum. Nature, 555(7694):67-70, February 2018. 1.5.4, 3.4, 3.10

[25] Paolo Panci. 21-cm line anomaly: A brief status. July 2019. Link: https://arxiv.org/abs/1907.13384. 1.5.4

[26] Daniel E. Holz, Scott A. Hughes, and Bernard F. Schutz. Measuring cosmic distances with standard sirens. Physics Today, 71(12):34-40, December 2018. 1.5.6, 4.8

[27] Philip W. Anderson. Brainwashed by Feynman? Physics Today, 53(2):11-12, February 2000. 1.5.7

[28] Richard P. Feynman. Cargo Cult Science. Commencement address, California Institute of Technology, June 1974. Link: http://calteches.library.caltech.edu/51/2/CargoCult.htm. 1.7

[29] Nathalie Palanque-Delabrouille, Christophe Yeche, Julien Baur, et al. Neutrino masses and cosmology with Lyman-alpha forest power spectrum. Journal of Cosmology and Astroparticle Physics, 2015(11):011-011, November 2015. 2.4

[30] Eric Weisstein. Delta function. Wolfram MathWorld web page. Link: http://mathworld.wolfram.com/DeltaFunction.html. 2.7

[31] M. Aker, K. Altenmuller, M. Arenz, et al. An improved upper limit on the neutrino mass from a direct kinematic method by KATRIN. September 2019. Link: https://arxiv.org/abs/1909.06048. 2.14

[32] G. Drexlin. Direct neutrino mass measurement. TAUP 2019, September 2019. Link: http://wwwkam2.icrr.u-tokyo.ac.jp/indico/event/3/contribution/27. 2.14

[33] G. A. Gonzalez-Sprinberg and J. Vidal. Tau magnetic moment. Journal of Physics: Conference Series, 912(1):012001, 2017. 2.15

[34] Erika K. Carlson. Synopsis: New Physics Possibilities from Kaon Decay. Physics, February 2020. Link: https://physics.aps.org/synopsis-for/10.1103/PhysRevLett.124.071801. 2.17

[35] Teppei Kitahara, Takemichi Okui, Gilad Perez, et al. New Physics Implications of Recent Search for $\mathrm{KL} \rightarrow \pi 0 \mathrm{v \nu}^{-}$at KOTO. Physical Review Letters, 124(7), February 2020. 2.17 
[36] Anonymous. Content of the universe - pie chart. National Aeronautics and Space Administration, April 2013. Link: https://map.gsfc.nasa.gov/media/080998/index.html. 3.1, 3.3, 3.10, 3.10

[37] Rennan Barkana. Possible interaction between baryons and dark-matter particles revealed by the first stars. Nature, 555(7694):71-74, February 2018. 3.4

[38] Christopher Crockett. A black hole eruption marks the most powerful explosion ever spotted. ScienceNews, February 2020. Link: https://www.sciencenews.org/article/black-hole-eruption-marksmost-powerful-explosion-ever-spotted. 3.5

[39] S. Giacintucci, M. Markevitch, M. Johnston-Hollitt, et al. Discovery of a Giant Radio Fossil in the Ophiuchus Galaxy Cluster. The Astrophysical Journal, 891(1):1, February 2020. 3.5

[40] Mark P. Hertzberg. Structure Formation in the Very Early Universe. Physics, 13(26), February 2020. Link: https://physics.aps.org/articles/v13/16. 3.6, 3.6

[41] Ned Wright. Brief History of the Universe. UCLA web page, July 2004. Link:

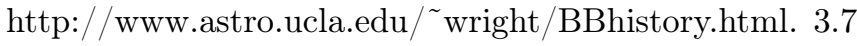

[42] Nick Gnedin. Cosmological Calculator for the Flat Universe, 2015. Link: http://home.fnal.gov/ gnedin/cc/. 3.8

[43] J. Jimenez-Vicente, E. Mediavilla, J. A. Munoz, and C. S. Kochanek. A ROBUST DETERMINATION OF THE SIZE OF QUASAR ACCRETION DISKS USING GRAVITATIONAL MICROLENSING. The Astrophysical Journal, 751(2):106, May 2012. 3.8

[44] Shannon Hall. Ghost galaxy is 99.99 per cent dark matter with almost no stars. New Scientist, August 2016. Link: https://www.newscientist.com/article/2102584-ghost-galaxy-is-99-99-per-centdark-matter-with-almost-no-stars/. 3.8

[45] Pavel E. Mancera Pina, Filippo Fraternali, Elizabeth A. K. Adams, et al. Off the baryonic tullyfisher relation: A population of baryon-dominated ultra-diffuse galaxies. The Astrophysical Journal, 883(2):L33, September 2019. 3.8

[46] Qi Guo, Huijie Hu, Zheng Zheng, et al. Further evidence for a population of dark-matter-deficient dwarf galaxies. Nature Astronomy, November 2019. 3.8

[47] Pieter van Dokkum, Shany Danieli, Yotam Cohen, et al. A galaxy lacking dark matter. Nature, 555(7698):629-632, March 2018. 3.8

[48] Ignacio Trujillo, Michael A. Beasley, Alejandro Borlaff, et al. A distance of $13 \mathrm{Mpc}$ resolves the claimed anomalies of the galaxy lacking dark matter. Monthly Notices of the Royal Astronomical Society, 486(1):1192-1219, June 2019. 3.8

[49] Pieter van Dokkum, Shany Danieli, Roberto Abraham, et al. A second galaxy missing dark matter in the NGC 1052 group. ApJ Letters, 874(1):L5, March 2019. 3.8

[50] Anonymous. Markarian 1216: Isolated galaxy has dark matter-rich core. Science News, June 2019. Link: http://www.sci-news.com/astronomy/markarian-1216-galaxy-dark-matter-rich-core07263.html. 3.8

[51] Ben Forrest, Marianna Annunziatella, Gillian Wilson, et al. An Extremely Massive Quiescent Galaxy at $\mathrm{z}=3.493$ : Evidence of Insufficiently Rapid Quenching Mechanisms in Theoretical Models. The Astrophysical Journal, 890(1):L1, February 2020. 3.8

[52] T. Wang, C. Schreiber, D. Elbaz, et al. A dominant population of optically invisible massive galaxies in the early universe. Nature, 572:211-214, August 2019. 3.8

[53] Heather Hill. Massive galaxies from the early universe found hiding in plain sight. Physics Today, September 2019. Link: https://physicstoday.scitation.org/do/10.1063/PT.6.1.20190909a/full/. 3.8

[54] Laura V. Sales, Julio F. Navarro, Louis Penafiel, et al. The Formation of Ultra-Diffuse Galaxies in Clusters. September 2019. Link: https://arxiv.org/abs/1909.01347. 3.8 
[55] M. Volonteri. Evolution of Supermassive Black Holes. In ESO Astrophysics Symposia, pages 174-182. Springer Berlin Heidelberg, 2007. 3.8

[56] Francesca Civano, Nico Cappelluti, Ryan Hickox, et al. Cosmic evolution of supermassive black holes: A view into the next two decades, March 2019. Link: https://arxiv.org/abs/1903.11091. 3.8

[57] Elizabeth Landau. Black hole seeds missing in cosmic garden. Jet Propulsion Laboratory News, September 2019. Link: https://www.jpl.nasa.gov/news/news.php?feature=7504. 3.8

[58] Duncan A Forbes, Adebusola Alabi, Aaron J Romanowsky, et al. Globular clusters in Coma cluster ultra-diffuse galaxies (UDGs): evidence for two types of UDG? Monthly Notices of the Royal Astronomical Society, 492(4):4874-4883, January 2020. 3.8

[59] David Ehrenstein. Mapping dark matter in the Milky Way. Physics, 12(51), May 2019. Link: https://physics.aps.org/articles/v12/51. 3.8

[60] Lina Necib, Mariangela Lisanti, and Vasily Belokurov. Dark matter in disequilibrium: The local velocity distribution from SDSS-Gaia. July 2018. Link: https://arxiv.org/abs/1807.02519v1. 3.8

[61] Ewa L. Lokas and Gary A. Mamon. Dark matter distribution in the coma cluster from galaxy kinematics: breaking the mass-anisotropy degeneracy. Monthly Notices of the Royal Astronomical Society, 343(2):401-412, August 2003. 3.9

[62] Elena Rasia, Giuseppe Tormen, and Lauro Moscardini. A dynamical model for the distribution of dark matter and gas in galaxy clusters. Monthly Notices of the Royal Astronomical Society, 351(1):237-252, June 2004. 3.9

[63] Lawrence Rudnick. The Stormy Life of Galaxy Clusters: astro version. January 2019. Link: https://arxiv.org/abs/1901.09448. 3.9

[64] Lawrence Rudnick. The stormy life of galaxy clusters. Physics Today, 72(1):46-52, January 2019. 3.9

[65] Man Ho Chan. A tight correlation between the enclosed gravitational mass and hot gas mass in galaxy clusters at intermediate radii. Physics of the Dark Universe, 28:100478, May 2020. 3.9

[66] G. Risaliti and E. Lusso. Cosmological constraints from the hubble diagram of quasars at high redshifts. Nature Astronomy, 3:272-277, January 2019. 3.10

[67] Feryal Ozel, Dimitrios Psaltis, Ramesh Narayan, and Antonio Santos Villarreal. ON THE MASS DISTRIBUTION AND BIRTH MASSES OF NEUTRON STARS. The Astrophysical Journal, 757(1):55, September 2012. 3.11

[68] Luciano Rezzolla, Elias R. Most, and Lukas R. Weih. Using Gravitational-wave Observations and Quasi-universal Relations to Constrain the Maximum Mass of Neutron Stars. The Astrophysical Journal, 852(2):L25, January 2018. 3.11

[69] Dana Najjar. 'Radical Change' Needed After Latest Neutron Star Collision. Quanta Magazine, February 2020. Link: https://www.quantamagazine.org/radical-change-needed-after-latest-neutronstar-collision-20200220/. 3.11, 3.11

[70] Mohammadtaher Safarzadeh, Enrico Ramirez-Ruiz, and Edo Berger. GW190425 is inconsistent with being a binary neutron star born from a fast merging channel. January 2020 . Link: https://arxiv.org/abs/2001.04502. 3.11

[71] Meinard Kuhlmann. Quantum Field Theory. In Edward N. Zalta, editor, The Stanford Encyclopedia of Philosophy. Metaphysics Research Lab, Stanford University, summer 2015 edition, 2015. Link: https://plato.stanford.edu/entries/quantum-field-theory/. 4.3

[72] Abhay Deshpande and Rikutaro Yoshida. The Experiment That Will Probe the Deepest Recesses of the Atom. Scientific American, 320(6), June 2019. 4.4

[73] Marric Stephens. Synopsis: How a pentaquark is put together. Physics, June 2019. Link: https://physics.aps.org/synopsis-for/10.1103/PhysRevLett.122.222001. 4.4, 5.4 
[74] Philip Ball. Quantum leaps, long assumed to be instantaneous, take time. Quanta magazine, July 2019. Link:https://www.quantamagazine.org/quantum-leaps-long-assumed-to-beinstantaneous-take-time-20190605/. 4.6

[75] Z. K. Minev, S. O. Mundhada, S. Shankar, P. Reinhold, et al. To catch and reverse a quantum jump mid-flight. Nature, 570(7760):20-204, June 2019. Link: https://www.nature.com/articles/s41586019-1287-z. 4.6

[76] R. Aaij, C. Abellan Beteta, B. Adeva, et al. Observation of a narrow pentaquark state, $P_{c}(4312)^{+}$, and of the two-peak structure of the $P_{c}(4450)^{+}$. Phys. Rev. Lett., 122:222001, June 2019. 5.4

[77] Sophie Ritson. The Many Dimensions of the String Theory Wars. PhD thesis, The University of Sydney, 2016. Link: https://www.academia.edu/37379424. 5.6

[78] Emily Conover. Giant radio bubbles spew from near the Milky Way's central black hole. Science News, September 2019. Link: https://www.sciencenews.org/article/radio-wave-bubbles-milky-wayblack-hole. 5.8

[79] I. Heywood, F. Camilo, W. D. Cotton, et al. Inflation of 430-parsec bipolar radio bubbles in the Galactic Centre by an energetic event. Nature, 573(7773):235-237, September 2019. Link: https://www.nature.com/articles/s41586-019-1532-5. 5.8

[80] J. Michael Roney. Viewpoint: CP Violations Newly Observed in Beauty Meson Decays. Physics, 13(6), January 2020. Link: https://physics.aps.org/articles/v13/6. 6.3

[81] Thomas J. Buckholtz. Models for Physics of the Very Small and Very Large, volume 14 of Atlantis Studies in Mathematics for Engineering and Science. Springer, 2016. Series editor: Charles K. Chui. 7

[82] Thomas J. Buckholtz. Predict particles beyond the standard model; then, narrow gaps between physics theory and data. In Proceedings of the 9th Conference on $N u$ clear and Particle Physics (19-23 Oct. 2015 Luxor-Aswan, Egypt), May 2016. Link: http://www.afaqscientific.com/nuppac15/npc1509.pdf. 7

This essay: Copyright (c) 2020 Thomas J. Buckholtz 